\title{
The association of smoking status with SARS-CoV-2 infection, hospitalisation and mortality from COVID-19: A living rapid evidence review with Bayesian meta-analyses (version 10)
}

\author{
David Simons ${ }^{1}$, Lion Shahab², Jamie Brown ${ }^{2}$, Olga Perski $^{2}$ \\ 1 Royal Veterinary College, RVC \\ 2 University College London, University of London
}

Funding: The author(s) received no specific funding for this work.

Potential competing interests: The author(s) declared that no potential competing interests exist.

\section{Abstract}

Aims: To estimate the association of smoking status with rates of i) infection, ii) hospitalisation, iii) disease severity, and iv) mortality from SARS-CoV-2/COVID-19 disease.

Design: Living rapid review of observational and experimental studies with random-effects hierarchical Bayesian metaanalyses. Published articles and pre-prints were identified via MEDLINE and medRxiv.

Setting: Community or hospital. No restrictions on location.

Participants: Adults who received a SARS-CoV-2 test or a COVID-19 diagnosis.

Measurements: Outcomes were SARS-CoV-2 infection, hospitalisation, disease severity and mortality stratified by smoking status. Study quality was assessed (i.e. 'good', 'fair' and 'poor').

Findings: Version 10 (searches up to 15 December 2020) included 345 studies with 52 'good' and 'fair' quality studies included in unadjusted meta-analyses. One-hundred-and-one studies (29.3\%) reported current, former and never smoking status with the remainder using broader categories. Recorded smoking prevalence among people with COVID19 was generally lower than national prevalence. Current compared with never smokers were at reduced risk of SARSCoV-2 infection $(R R=0.69,95 \%$ Credible Interval $(\mathrm{Crl})=0.58-0.82, \tau=0.36)$. Data for former smokers were inconclusive $(\mathrm{RR}=1.03,95 \% \mathrm{Crl}=0.94-1.13, \tau=0.18)$ but favoured there being no important association $(8 \%$ probability of $R R \geq 1.1)$. Former compared with never smokers were at increased risk of hospitalisation $(R R=1.18, \mathrm{Crl}=$ 1.07-1.31, $\tau=0.14)$, greater disease severity $(R R=1.52, \mathrm{Crl}=1.12-2.06, \tau=0.29)$ and mortality $(R R=1.40,95 \% \mathrm{Crl}=$ 1.20-1.64, $\tau=0.19)$. Data for current smokers on hospitalisation, disease severity and mortality were inconclusive $(R R=$ 
1.08, $\mathrm{Crl}=0.95-1.23, \tau=0.18 ; \mathrm{RR}=1.26, \mathrm{Crl}=0.85-1.93, \tau=0.34 ; \mathrm{RR}=1.05,95 \% \mathrm{Crl}=0.77-1.41, \tau=0.39$, respectively) but favoured there being no important associations with hospitalisation and mortality $(31 \%$ and $38 \%$ probability of $R R \geq 1.1$, respectively) and a small but important association with disease severity (80\% probability of RR $\geq 1.1)$.

Conclusions: Compared with never smokers, current smokers appear to be at reduced risk of SARS-CoV-2 infection while former smokers appear to be at increased risk of hospitalisation, greater disease severity and mortality from COVID-19. However, it is uncertain whether these associations are causal.

v7 of this living review article has been published in Addiction and is available here https://doiorg.libproxy.ucl.ac.uk/10.1111/add.15276

\section{Definitions}

Angiotensin-Converting Enzyme

Defined by National Cancer Institute

SARS Coronavirus

Defined by National Cancer Institute

Cigarette Smoker

Defined by National Cancer Institute

Nicotine

Defined by National Cancer Institute

Inflammation

Defined by National Cancer Institute

Fibrosis

Defined by National Cancer Institute

Living Systematic Review

Defined by Julian H. Elliott et al.

Randomized controlled trials

Defined by David F Marks

Meta-analysis

Defined by Arindam Basu

Bayesian Approach

Defined by National Cancer Institute

\section{Introduction}

COVID-19 is a respiratory disease caused by the SARS-CoV-2 virus. Large age and gender differences in case severity and mortality have been observed in the ongoing COVID-19 pandemic ${ }^{1}$; however, these differences are currently 
unexplained. SARS-CoV-2 enters epithelial cells through the angiotensin-converting enzyme 2 (ACE-2) receptor ${ }^{2}$. Some evidence suggests that gene expression and subsequent receptor levels are elevated in the airway and oral epithelium of current smokers ${ }^{3,4}$, which could put smokers at higher risk of contracting SARS-CoV-2. Other studies, however, suggest that nicotine downregulates the ACE-2 receptor ${ }^{5}$. These uncertainties notwithstanding, both former and current smoking is known to increase the risk of respiratory viral $\left.\right|^{6,7}$ and bacterial $\left.\right|^{8,9}$ infections and is associated with worse outcomes once infected. Cigarette smoke reduces the respiratory immune defence through peri-bronchiolar inflammationand fibrosis, impaired mucociliary clearance and disruption of the respiratory epithelium ${ }^{10}$. There is also reason to believe that behavioural factors (e.g. regular hand-to-mouth movements) involved in smoking may increase SARS-CoV-2 infection and transmission in current smokers. However, early data from the COVID-19 pandemic have not provided clear evidence for a negative impact of current or former smoking on SARS-CoV-2 infection or COVID-19 disease outcomes, such as hospitalisation or mortality ${ }^{11}$. It has also been hypothesised that nicotine might protect against a hyper-inflammatory response to SARS-CoV-2 infection, which may lead to adverse outcomes in patients with COVID-19 disease ${ }^{12}$.

There are several reviews that fall within the scope of smoking and COVID-1911,13-18. We aimed to produce a rapid synthesis of available evidence pertaining to the rates of infection, hospitalisation, disease severity and mortality from SARS-CoV-2/COVID-19 stratified by smoking status. Given the increasing availability of data on this topic, this is a living review with regular updates. As evidence accumulates, the review will be expanded to include studies reporting COVID-19 outcomes by alternative nicotine use (e.g., nicotine replacement therapy or e-cigarettes).

\section{Methods}

\section{Study design}

This is a living evidence review which is updated as new evidence becomes available ${ }^{19}$. We adopted recommended best practice for rapid evidence reviews, which involved limiting the search to main databases and having one reviewer extract the data and another verify ${ }^{20}$. This study was not pre-registered but evolved from a report written for a UK medical society ${ }^{21}$. The most recent (and all future) version(s) of this living review is available here (https://www.qeios.com/read/latest-UJR2AW). Version 7 of this living review has been published in a peer-reviewed journal22. A completed Preferred Reporting Items for Systematic Reviews and Meta-Analyses (PRISMA) checklist is included in Supplementary file 1.

\section{Eligibility criteria}

Studies were included if they:

1. Were primary research studies using experimental (e.g. randomised controlled trial), quasi-experimental (e.g. pre- and post-test) or observational (e.g. case-control, retrospective cohort, prospective cohort) study designs; 
2. Included adults aged $16+$ years;

3. Recorded as outcome i) results of a SARS-CoV-2 diagnostic test (including antibody assays), ii) clinical diagnosis of COVID-19, iii) hospitalisation with COVID-19, iv) severity of COVID-19 disease in those hospitalised or v) mortality from COVID-19;

4. Reported any of the outcomes of interest by self-reported or biochemically verified smoking status (e.g. current smoker, former smoker, never smoker) or current vaping and nicotine replacement therapy (NRT) use;

5. Were available in English;

6. Were published in a peer-reviewed journal, as a pre-print or a public health report by reputable bodies (e.g. governments, scientific societies).

\section{Search strategy}

The following terms were searched for in Ovid MEDLINE (2019-search date) as free text or Medical Subject Headings:

1. Tobacco Smoking/ or Smoking Cessation/ or Water Pipe Smoking/ or Smoking/ or Smoking Pipes/ or Cigar Smoking/ or Smoking Prevention/ or Cigarette Smoking/ or smoking.mp. or Pipe Smoking/ or Smoking, Non-Tobacco Products/ or Smoking Water Pipes/

2. Nicotine/ or nicotine.mp. or Electronic Nicotine Delivery Systems/ or Nicotine Chewing Gum/

3. vaping.mp. or Vaping/

4. 1 or 2 or 3

5. Coronavirus/ or Severe Acute Respiratory Syndrome/ or Coronavirus Infections/ or covid.mp.

6. 4 and 5

The following terms were searched for in titles, abstracts and full texts in medRxiv (no time limitations):

1. covid (this term captures both covid and SARS-CoV-2) AND smoking

2. covid AND nicotine

3. covid AND vaping

Additional articles/reports of interest were identified through mailing lists, Twitter, the International Severe Acute

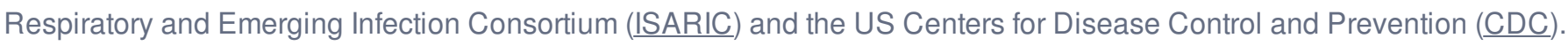
Where updated versions of pre-prints or public health reports were available, old versions were superseded.

\section{Selection of studies}

One reviewer screened titles, abstracts and full texts against the inclusion criteria. 


\section{Data extraction}

Data were extracted by one reviewer and verified (i.e. independently checked against pre-prints and published reports) by another on i) author (year); ii) date published; iii) country; iv) study design; v) study setting; vi) sample size; vii) sex; viii) age; ix) smoking status (e.g. current, former, never, not stated, missing) and whether it was biochemically verified; $x$ ) use of alternative nicotine products; xi) SARS-CoV-2 testing; xii) SARS-CoV-2 infection; xiii) diagnosis of COVID-19; xiv) hospitalisation with COVID-19; xv) disease severity in those hospitalised with COVID-19; xvi) mortality; xvii) adjustment of smoking specific risk estimates for relevant covariates (e.g. age, sex); and xviii) whether a representative or random sampling method was used.

\section{Quality appraisal}

The quality of included studies was assessed to determine suitability for inclusion in meta-analyses. Studies were judged as 'good' quality if they: i) had $<20 \%$ missing data on smoking status and used a reliable self-report measure that distinguished between current, former and never smoking status; AND ii) used biochemical verification of smoking status and reported results from adjusted analyses; OR reported data from a representative/random sample. Studies were rated as 'fair' if they fulfilled only criterion i) and were otherwise rated as 'poor'. The quality appraisal was conducted by one reviewer and verified by a second.

\section{Evidence synthesis}

A narrative synthesis was conducted. Data from 'good' and 'fair' quality studies were pooled in R v.3.6.3 23 . In a living review where new data are regularly added to the analyses, it may be more appropriate to use a Bayesian (as opposed to frequentist) approach where prior knowledge is used in combination with new data to estimate a posterior risk distribution. A Bayesian approach mitigates the issue of performing multiple statistical tests, which can inflate family-wise error. A series of random-effects hierarchical Bayesian meta-analyses were performed with the $b r m s^{24}$ package to estimate the relative risk for each comparison with accompanying 95\% credible intervals (Crls). We first defined prior distributions for the true pooled effect size $(\mu)$ and the between-study heterogeneity $(\tau)$, with $\mu$ specified as a normal distribution with a mean equal to the derived point estimate from each comparison of interest in the immediately preceding version of this living review ${ }^{25}$, and $\tau$ specified as a half-Cauchy distribution with a mean of 0 and standard deviation of 1 . The halfCauchy distribution was selected to reflect prior knowledge that high levels of between-study heterogeneity are more likely than lower levels. Markov Chain Monte Carlo methods (20,000 burn-ins followed by 80,000 iterations) were then used to generate a risk distribution for each study, in addition to a pooled effect for the posterior risk distribution. We report forest plots with the pooled effect for the posterior risk distribution displayed as the median relative risk with an accompanying 95\% Crls. We used the empirical cumulative distribution function (ECDF) to estimate the probability of there being a $10 \%$ reduction or $10 \%$ increase in relative risk ( $R R$ ) (i.e. $R R \geq 1.1$ or $R R \leq 0.9$ ). Due to a lack of indication as to what constitutes a clinically or epidemiologically meaningful effect (e.g. with regards to onward disease transmission or requirements for 
intensive care beds), we deemed a 10\% change in risk as small but important. Where data were inconclusive (as indicated by Crls crossing $R R=1.0$ ), to disambiguate whether data favoured no effect or there being a small but important association, we estimated whether there was $\geq 75 \%$ probability of $R R \geq 1.1$ or $R R \leq 0.9$.

Two sensitivity analyses were performed. First, a minimally informative prior for $\mu$ was specified as a normal distribution with a mean of 0 and standard deviation of 1 and $\tau$ as described above. Second, an informative prior as described above for $\mu$ was used with $\tau$ specified as a half-Cauchy distribution with a mean of 0.3 and standard deviation of 1 to reflect greater between-study heterogeneity.

To aid in the visualisation of smoking prevalence in the included studies, the weighted mean prevalence of current and former smoking was calculated for countries with $\geq 3$ studies and plotted for comparison with national prevalence estimates. It should be noted that prevalence estimates in the included studies were not adjusted for age, sex, socioeconomic position, or geographic region within countries.

\section{Results}

In the current review version (v10) with searches up to 15 December 2020, a total of 874 records were identified, with 345 studies included in a narrative synthesis and 52 studies included in meta-analyses (see Figure 1).

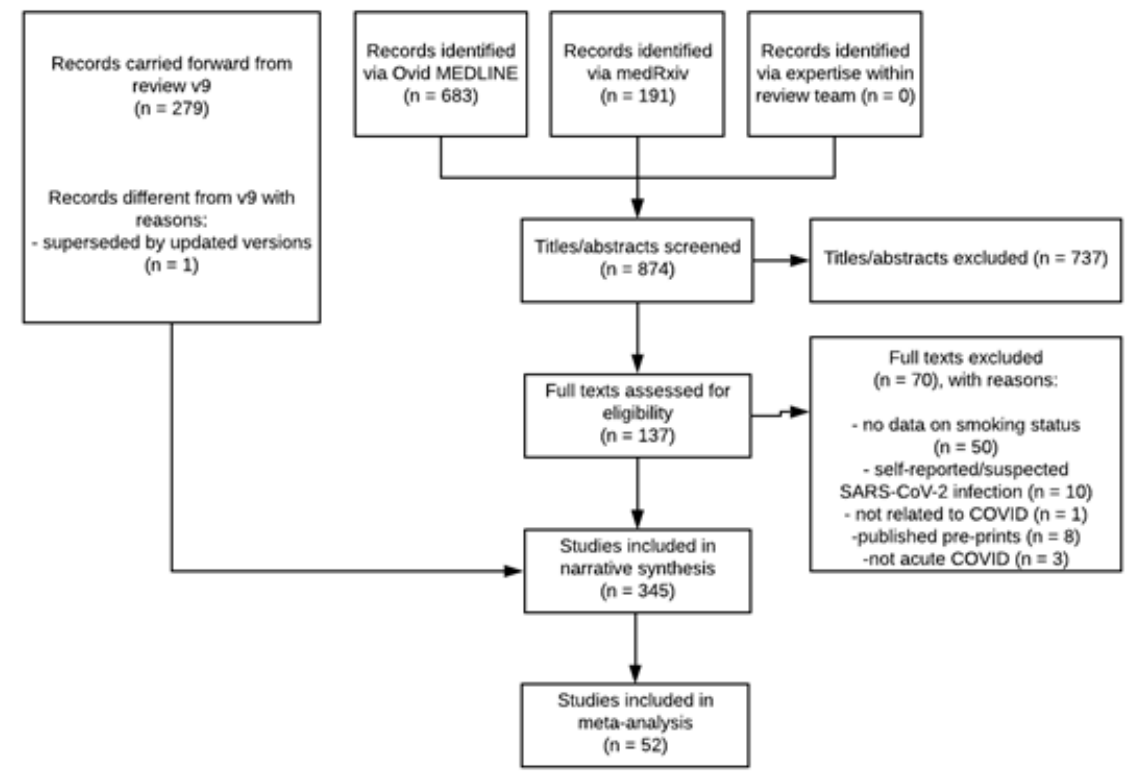

Figure 1. PRISMA flow diagram of included studies.

\section{Study characteristics}

Characteristics of included studies are presented in Table 1. Studies were conducted across 39 countries. Ninety-three 
studies were conducted in the US, 65 in China, 36 in the UK, 24 in Spain, 18 in France, 17 in Mexico, 13 in Italy, 10 across multiple international sites, six in Brazil, five in Iran and Israel, four in Turkey, three in Australia, Finland, Saudi Arabia and Switzerland, two in Austria, Bangladesh, Chile, Colombia, Denmark, Egypt, Germany, India, Japan, Kuwait, the Netherlands, Qatar and South Korea, with one each from 11 further countries. The majority of studies used observational designs (see Supplementary table S1). Two-hundred-and-twenty-four studies (65\%) were conducted in hospital settings, 91 studies (26\%) included a community component in addition to hospitalised patients, 27 studies (8\%) were conducted exclusively in the community, with one study each conducted in a homeless shelter and a quarantine centre, and one study that did not state the study setting. Studies had a median of 457 (interquartile range $=134-1,822$ ) participants. The majority of studies (82\%) used reverse transcriptase polymerase chain reaction (RT-PCR) for confirmation of SARS-CoV-2 infection, $5.6 \%$ used an antibody test to confirm prior infection and $4 \%$ of studies relied on a combination of RT-PCR or antibody assays.

\section{Smoking status}

Categorisation of smoking status was heterogeneous (see Table 1). Two-hundred-and-eleven studies collected data on smoking status through routine electronic health records (EHRs), 100 studies used a bespoke case report form for COVID19 and 34 studies did not state the source for information on smoking status. None of the studies verified smoking status biochemically. Notably, only 101 (29.3\%) studies reported current, former and never smoking status (see Supplementary table S2a), with a further 26 studies reporting only ever and never smoking status (see Supplementary table S2b). The remaining 218 studies reported current, current/former or current and former smoking status but did not explicitly state whether remaining participants were never smokers or if data were missing on smoking status (see Supplementary table S2c). One-hundred-and-seventeen studies explicitly reported the proportion with missing data on smoking status, which ranged from $0 \%$ to $96.4 \%$.

\section{Use of alternative nicotine products}

Six studies recorded the use of alternative nicotine products in current and/or former smokers but did not report COVID-19 outcomes stratified by alternative nicotine use $\mathrm{e}^{26-31}$.

\section{Quality appraisal}

Two studies were performed in random, representative population samples and were rated as 'good' quality, and 77 studies were rated as 'fair' quality, of which 52 studies reported results stratified by smoking status for the outcomes of interest and could be included in meta-analyses. The remaining 266 studies were rated as 'poor' quality (see Table 1).

Table 1. Characteristics of included studies. 


\begin{tabular}{|c|c|c|c|c|c|c|c|c|c|c|c|c|}
\hline Ref. & Lead author & $\begin{array}{l}\text { Date } \\
\text { published }\end{array}$ & Country & $\begin{array}{l}\text { Sample } \\
\text { size }\end{array}$ & $\begin{array}{l}\text { Study } \\
\text { setting }\end{array}$ & $\begin{array}{l}\text { Median } \\
(I Q R)\end{array}$ & $\begin{array}{l}\text { Female } \\
\%\end{array}$ & $\begin{array}{l}\text { smoker } \\
\%\end{array}$ & $\begin{array}{l}\text { smokers } \\
\%\end{array}$ & $\begin{array}{l}\text { Current/former } \\
\text { smokers \% }\end{array}$ & $\begin{array}{l}\text { smokers } \\
\%\end{array}$ & $\begin{array}{l}\text { Never/unk } \\
\text { smokers c }\end{array}$ \\
\hline 1 & Guan, Ni & $\begin{array}{l}2020-02- \\
28\end{array}$ & China & 1,099 & Hospital & $\begin{array}{c}47 \\
(35-58)\end{array}$ & 41.9 & 12.5 & 1.9 & - & 84.3 & - \\
\hline 59 & Guan, Liang & $\begin{array}{l}2020-03- \\
26\end{array}$ & China & 1,590 & Hospital & $\begin{array}{c}49 \\
(33-64)\end{array}$ & 42.7 & - & - & 7.0 & 93.0 & - \\
\hline 60 & Lian & $\begin{array}{l}2020-03- \\
25\end{array}$ & China & 788 & Hospital & NA & 38.5 & 6.9 & - & - & - & - \\
\hline 61 & Jin & $\begin{array}{l}2020-03- \\
24\end{array}$ & China & 651 & Hospital & $\begin{array}{c}46 \\
(32-60)\end{array}$ & 49.2 & 6.3 & - & - & - & - \\
\hline 62 & Chen & $\begin{array}{l}2020-03- \\
26\end{array}$ & China & 548 & Hospital & $\begin{array}{c}62 \\
(44-70)\end{array}$ & 37.6 & 4.4 & 2.6 & - & - & - \\
\hline 63 & Zhou, Yu & $\begin{array}{l}2020-03- \\
11\end{array}$ & China & 191 & Hospital & $\begin{array}{c}56 \\
(46-67)\end{array}$ & 38.0 & 5.8 & - & - & - & - \\
\hline 64 & Mo & $\begin{array}{l}2020-03- \\
16\end{array}$ & China & 155 & Hospital & $\begin{array}{c}54 \\
(53-66)\end{array}$ & 44.5 & 3.9 & - & - & - & - \\
\hline 65 & Zhang, Dong & $\begin{array}{l}2020-02- \\
19\end{array}$ & China & 140 & Hospital & $\begin{array}{c}57^{\wedge} \\
(25-87)\end{array}$ & 46.3 & 1.4 & 5.0 & - & - & - \\
\hline 66 & Wan & $\begin{array}{l}2020-03- \\
21\end{array}$ & China & 135 & Hospital & $\begin{array}{c}47 \\
(36-55)\end{array}$ & 46.7 & 6.7 & - & - & - & - \\
\hline 67 & Liu, Tao & $\begin{array}{l}2020-02- \\
28\end{array}$ & China & 78 & Hospital & $\begin{array}{c}38 \\
(33-57)\end{array}$ & 50.0 & - & - & 6.4 & - & - \\
\hline 68 & Huang, Wang & $\begin{array}{l}2020-01- \\
24\end{array}$ & China & 41 & Hospital & $\begin{array}{c}49 \\
(41-58)\end{array}$ & 27.0 & 7.3 & - & - & - & - \\
\hline 69 & Zhang, Cai & $\begin{array}{l}2020-03- \\
20\end{array}$ & China & 645 & Hospital & NA & 49.1 & 6.4 & - & - & - & - \\
\hline 70 & Guo & $\begin{array}{l}2020-03- \\
27\end{array}$ & China & 187 & Hospital & $\begin{array}{c}59 \\
(45-73)\end{array}$ & 51.3 & 9.6 & - & - & - & - \\
\hline 71 & Liu, Ming & $\begin{array}{l}2020-03- \\
12\end{array}$ & China & 41 & Hospital & $\begin{array}{c}39 \\
(30-48)\end{array}$ & 58.5 & 9.8 & - & - & - & - \\
\hline 72 & Huang, Yang & $\begin{array}{l}2020-03- \\
05\end{array}$ & China & 36 & Hospital & $\begin{array}{c}69 \\
(60-78)\end{array}$ & 30.6 & - & - & 11.1 & - & - \\
\hline 73 & $\mathrm{Xu}$ & $\begin{array}{l}2020-03- \\
08\end{array}$ & China & 53 & Hospital & NA & 47.2 & 11.3 & - & - & - & - \\
\hline 74 & $\mathrm{Li}$ & $\begin{array}{l}2020-02- \\
12\end{array}$ & China & 17 & Hospital & $\begin{array}{c}45 \\
(33-57)\end{array}$ & 47.1 & 17.6 & - & - & - & - \\
\hline 32 & Rentsch & $\begin{array}{l}2020-04- \\
14\end{array}$ & USA & 3,528 & $\begin{array}{l}\text { Community } \\
\text { and } \\
\text { Hospital }\end{array}$ & $\begin{array}{c}66 \\
(60-70)\end{array}$ & 4.6 & 27.2 & 30.6 & - & 36.9 & - \\
\hline 75 & $\mathrm{Hu}$ & $\begin{array}{l}2020-03- \\
25\end{array}$ & China & 323 & Hospital & $\begin{array}{c}61^{\wedge} \\
(23-91)\end{array}$ & 48.6 & - & - & 11.8 & - & - \\
\hline 76 & Wang, Pan & $\begin{array}{l}2020-03- \\
24\end{array}$ & China & 125 & Hospital & $\begin{array}{c}41 \\
(26-66)\end{array}$ & 43.2 & - & - & 12.8 & - & - \\
\hline 77 & $\begin{array}{l}\text { Chow (US } \\
\text { CDC) }\end{array}$ & $\begin{array}{l}2020-03- \\
31\end{array}$ & USA & 7,162 & $\begin{array}{l}\text { Community } \\
\text { and } \\
\text { Hospital }\end{array}$ & NA & - & 1.3 & 2.3 & - & - & - \\
\hline 78 & Dong, Cao & $\begin{array}{l}2020-03- \\
20\end{array}$ & China & 9 & Hospital & $\begin{array}{c}44 \\
(30-46)\end{array}$ & 66.7 & 11.1 & - & - & - & - \\
\hline 79 & Kim & $\begin{array}{l}2020-04- \\
01\end{array}$ & $\begin{array}{l}\text { South } \\
\text { Korea }\end{array}$ & 28 & Hospital & $\begin{array}{c}43 \\
(30-56)\end{array}$ & 46.4 & 17.9 & - & - & - & - \\
\hline
\end{tabular}




\begin{tabular}{|c|c|c|c|c|c|c|c|c|c|c|c|c|}
\hline & -..., , u & 18 & c...... & . & muritur & $(27-65)$ & .u. & & & $u \cdot$ & & \\
\hline 81 & Yang, Yu & $\begin{array}{l}2020-02- \\
24\end{array}$ & China & 52 & Hospital & $\begin{array}{c}60 \\
(47-73)\end{array}$ & 37.0 & 3.8 & - & - & - & - \\
\hline 82 & Argenziano & $\begin{array}{l}2020-05- \\
29\end{array}$ & USA & 1,000 & Hospital & $\begin{array}{c}63 \\
(50-75)\end{array}$ & 40.4 & 4.9 & 17.9 & - & 77.2 & - \\
\hline 83 & Solis & $\begin{array}{l}2020-04- \\
25\end{array}$ & Mexico & 650 & Hospital & ${ }{ }^{46}{ }^{46}$ & 42.1 & 9.4 & - & - & - & - \\
\hline 84 & Richardson & $\begin{array}{l}2020-04- \\
22\end{array}$ & USA & 5,700 & Hospital & $\begin{array}{c}63 \\
(52-75)\end{array}$ & 39.7 & - & - & 9.8 & 52.8 & - \\
\hline 85 & Fontanet & $\begin{array}{l}2020-04- \\
23\end{array}$ & France & 661 & $\begin{array}{l}\text { Community } \\
\text { and } \\
\text { Hospital }\end{array}$ & $\begin{array}{c}37 \\
(16-47)\end{array}$ & 62.0 & 10.4 & - & - & - & 89.6 \\
\hline 87 & Liao, Feng & $\begin{array}{l}2020-04- \\
24\end{array}$ & China & 1,848 & Hospital & $\begin{array}{c}55 \\
(48-61)\end{array}$ & 54.7 & - & - & 0.4 & - & - \\
\hline 88 & Gil-Agudo & $\begin{array}{l}2020-04- \\
24\end{array}$ & Spain & 7 & Hospital & $\begin{array}{c}68 \\
(34-75)\end{array}$ & 28.6 & - & - & 42.9 & 57.1 & - \\
\hline 89 & Shi, Ren & $\begin{array}{l}2020-04- \\
23\end{array}$ & China & 134 & Hospital & $\begin{array}{c}46 \\
(34-58)\end{array}$ & 51.5 & - & - & 10.4 & - & - \\
\hline 90 & Hadjadj & $\begin{array}{l}2020-04- \\
23\end{array}$ & France & 50 & Hospital & $\begin{array}{c}55 \\
(50-63)\end{array}$ & 22.0 & 2.0 & 18.0 & - & 80.0 & - \\
\hline 91 & Gold (US CDC) & $\begin{array}{l}2020-04- \\
20\end{array}$ & USA & 305 & Hospital & NA & 50.5 & 5.2 & - & - & - & - \\
\hline 93 & Zheng, Xiong & $\begin{array}{l}2020-04- \\
30\end{array}$ & China & 73 & Hospital & $\begin{array}{l}43^{\wedge} \\
(\mathrm{NA})\end{array}$ & 45.2 & - & - & 11.0 & 89.0 & - \\
\hline 94 & de la Rica & $\begin{array}{l}2020-05- \\
11\end{array}$ & Spain & 48 & Hospital & $\begin{array}{c}66^{\wedge} \\
(33-88)\end{array}$ & 33.0 & - & - & 20.8 & - & - \\
\hline 95 & Yin, Yang & $\begin{array}{l}2020-05- \\
10\end{array}$ & China & 106 & Hospital & $\begin{array}{c}73 \\
(61-85)\end{array}$ & 39.6 & - & - & 17.0 & - & - \\
\hline 96 & Shi, Zuo & $\begin{array}{l}2020-05- \\
17\end{array}$ & USA & 172 & Hospital & $\begin{array}{c}63^{\wedge} \\
(44-82)\end{array}$ & 44.0 & - & - & 26.2 & - & - \\
\hline 97 & Cho & $\begin{array}{l}2020-05- \\
11\end{array}$ & UK & 322,341 & $\begin{array}{l}\text { Community } \\
\text { and } \\
\text { Hospital }\end{array}$ & NA & 49.2 & 14.2 & 21.4 & - & 64.4 & - \\
\hline 98 & Allenbach & $\begin{array}{l}2020-05- \\
08\end{array}$ & France & 152 & Hospital & $\begin{array}{c}77 \\
(60-83)\end{array}$ & 31.1 & - & - & 6.6 & - & - \\
\hline 99 & Robilotti & $\begin{array}{l}2020-05- \\
08\end{array}$ & USA & 423 & Hospital & NA & 50.0 & 2.1 & 37.6 & - & 58.6 & - \\
\hline 100 & $\begin{array}{l}\text { The } \\
\text { Opensafely } \\
\text { Collaborative }\end{array}$ & $\begin{array}{l}2020-07- \\
01\end{array}$ & UK & $17,278,392$ & $\begin{array}{l}\text { Community } \\
\text { and } \\
\text { Hospital }\end{array}$ & NA & 50.1 & 17.0 & 32.9 & - & 45.9 & - \\
\hline 101 & Borobia & $\begin{array}{l}2020-05- \\
06\end{array}$ & Spain & 2,226 & Hospital & $\begin{array}{c}61 \\
(46-78)\end{array}$ & 52.0 & 7.1 & - & - & - & - \\
\hline 102 & Giacomelli & $\begin{array}{l}2020-05- \\
06\end{array}$ & Italy & 233 & Hospital & $\begin{array}{c}61 \\
(50-72)\end{array}$ & 31.9 & - & - & 30.0 & 70.0 & - \\
\hline
\end{tabular}




\begin{tabular}{|c|c|c|c|c|c|c|c|c|c|c|c|c|}
\hline 104 & Kolin & $\begin{array}{l}2020-05- \\
05\end{array}$ & UK & 502,536 & $\begin{array}{l}\text { Community } \\
\text { and } \\
\text { Hospital }\end{array}$ & $\begin{array}{c}56.5 \\
(48-64)\end{array}$ & 54.4 & 10.5 & 34.4 & - & 54.4 & - \\
\hline 105 & Lubetzky & $\begin{array}{l}2020-05- \\
08\end{array}$ & USA & 54 & Hospital & $\begin{array}{c}57 \\
(29-83)\end{array}$ & 62.0 & - & - & 22.2 & - & - \\
\hline 106 & Goyal & $\begin{array}{l}2020-04- \\
17\end{array}$ & USA & 393 & Hospital & $\begin{array}{c}62.2 \\
(49-74)\end{array}$ & 39.3 & 5.1 & - & - & - & - \\
\hline 107 & Feng & $\begin{array}{l}2020-04- \\
10\end{array}$ & China & 476 & Hospital & $\begin{array}{c}53 \\
(40-64)\end{array}$ & 43.1 & 9.2 & - & - & - & - \\
\hline 108 & Yao & $\begin{array}{l}2020-04- \\
24\end{array}$ & China & 108 & Hospital & $\begin{array}{c}52 \\
(37-58)\end{array}$ & 60.2 & 3.7 & - & - & - & - \\
\hline 109 & Sami & $\begin{array}{l}2020-05- \\
19\end{array}$ & Iran & 490 & Hospital & $\begin{array}{r}56.6 \\
(41-71)\end{array}$ & 39.0 & 14.1 & - & - & - & 85.9 \\
\hline 110 & Almazeedi & $\begin{array}{l}2020-05- \\
15\end{array}$ & Kuwait & 1,096 & Hospital & $\begin{array}{c}41 \\
(25-57)\end{array}$ & 19.0 & 4.0 & - & - & - & 96.0 \\
\hline 111 & Carillo-Vega & $\begin{array}{l}2020-05- \\
14\end{array}$ & Mexico & 10,544 & $\begin{array}{l}\text { Community } \\
\text { and } \\
\text { Hospital }\end{array}$ & $\begin{array}{r}46.5^{\wedge} \\
(30-62)\end{array}$ & 42.3 & 8.9 & - & - & - & - \\
\hline 112 & Yanover & $\begin{array}{l}2020-05- \\
13\end{array}$ & Israel & 4,353 & $\begin{array}{l}\text { Community } \\
\text { and } \\
\text { Hospital }\end{array}$ & $\begin{array}{c}35 \\
(22-54)\end{array}$ & 44.5 & 11.8 & 3.0 & - & 85.2 & - \\
\hline 113 & Hamer & $\begin{array}{l}2020-05- \\
13\end{array}$ & UK & 387,109 & Hospital & $\begin{array}{r}56.2 \\
(48-64)\end{array}$ & 55.1 & 9.7 & 34.8 & - & 55.5 & - \\
\hline 114 & Regina & $\begin{array}{l}2020-05- \\
14\end{array}$ & Switzerland & 200 & Hospital & $\begin{array}{c}70 \\
(55-81)\end{array}$ & 40.0 & 4.5 & - & - & - & - \\
\hline 116 & Targher & $\begin{array}{l}2020-05- \\
13\end{array}$ & China & 339 & Hospital & $\begin{array}{l}48.4^{\wedge} \\
\text { (NA) }\end{array}$ & 52.8 & 8.3 & - & - & - & - \\
\hline 117 & Valenti & $\begin{array}{l}2020-05- \\
18\end{array}$ & Italy & 789 & Community & $\begin{array}{l}40.7^{\wedge} \\
\text { (NA) }\end{array}$ & 35.0 & 25.9 & - & - & - & - \\
\hline 118 & Feuth & $\begin{array}{l}2020-05- \\
18\end{array}$ & Finland & 28 & Hospital & $\begin{array}{c}56 \\
(47-72)\end{array}$ & 46.0 & 10.7 & 28.6 & - & 60.7 & - \\
\hline 119 & $\mathrm{Ge}$ & $\begin{array}{l}2020-05- \\
18\end{array}$ & China & 51 & Hospital & $\begin{array}{c}70 \\
(58-79)\end{array}$ & 27.5 & 13.7 & - & - & - & - \\
\hline 120 & Parrotta & $\begin{array}{l}2020-05- \\
18\end{array}$ & USA & 76 & $\begin{array}{l}\text { Community } \\
\text { and } \\
\text { Hospital }\end{array}$ & $\begin{array}{r}44.9 \\
(13-71)\end{array}$ & 61.8 & 2.6 & 26.3 & - & 68.4 & - \\
\hline 121 & Shekhar & $\begin{array}{l}2020-05- \\
18\end{array}$ & USA & 50 & Hospital & $\begin{array}{c}55.5 \\
(20-85)\end{array}$ & 54.0 & 48.0 & - & - & - & - \\
\hline 122 & Mejia-Vilet & $\begin{array}{l}2020-05- \\
16\end{array}$ & Mexico & 329 & Hospital & $\begin{array}{c}49 \\
(41-60)\end{array}$ & 36.0 & - & - & 7.0 & - & - \\
\hline 123 & Chen, Jiang & $\begin{array}{l}2020-05- \\
16\end{array}$ & China & 135 & Hospital & NA & 42.2 & - & - & 9.6 & - & - \\
\hline 124 & Li, Chen & $\begin{array}{l}2020-05- \\
16\end{array}$ & China & 1,008 & Hospital & $\begin{array}{c}55 \\
(44-65)\end{array}$ & 43.6 & 5.7 & - & - & - & - \\
\hline 27 & Rimland & $\begin{array}{l}2020-05- \\
19\end{array}$ & USA & 11 & Hospital & $\begin{array}{c}59 \\
(48-65)\end{array}$ & 18.2 & 9.1 & - & - & - & - \\
\hline
\end{tabular}




\begin{tabular}{|c|c|c|c|c|c|c|c|c|c|c|c|c|}
\hline 125 & Palaiodimos & $\begin{array}{l}\text { cucu-uu- } \\
15\end{array}$ & USA & 200 & Hospital & $\begin{array}{l}(50- \\
73.5)\end{array}$ & 51.0 & - & - & 32.5 & 67.5 & - \\
\hline 126 & Ip & $\begin{array}{l}2020-05- \\
25\end{array}$ & USA & 2,512 & Hospital & $\begin{array}{c}64 \\
(52-76)\end{array}$ & 37.6 & 3.1 & 17.8 & - & 64.5 & - \\
\hline 127 & Heili-Frades & $\begin{array}{l}2020-05- \\
25\end{array}$ & Spain & 4,712 & Hospital & $\begin{array}{c}62 \\
(47-77)\end{array}$ & 50.5 & 4.9 & 17.4 & - & - & 66.5 \\
\hline 128 & $\begin{array}{l}\text { Vaquero- } \\
\text { Roncero }\end{array}$ & $\begin{array}{l}2020-05- \\
24\end{array}$ & Spain & 146 & Hospital & $\begin{array}{c}66^{\wedge} \\
(59-72)\end{array}$ & 32.2 & - & - & 6.8 & - & - \\
\hline 129 & Kim, Garg & $\begin{array}{l}2020-05- \\
22\end{array}$ & USA & 2,491 & Hospital & $\begin{array}{c}62 \\
(50-75)\end{array}$ & 46.8 & 6.0 & 25.8 & - & - & 68.1 \\
\hline 130 & Wu & $\begin{array}{l}2020-05- \\
21\end{array}$ & Italy & 174 & Hospital & $\begin{array}{c}61.2^{\wedge} \\
(50-71)\end{array}$ & 30.5 & - & - & 33.3 & - & - \\
\hline 131 & Shi, Zhao & $\begin{array}{l}2020-05- \\
20\end{array}$ & China & 101 & Hospital & $\begin{array}{c}71 \\
(59-80)\end{array}$ & 40.6 & - & - & 5.0 & - & - \\
\hline 132 & Al-Hindawi & $\begin{array}{l}2020-05- \\
20\end{array}$ & UK & 31 & Hospital & (NA) $^{61}$ & 12.9 & 3.2 & 71.0 & - & 25.8 & - \\
\hline 133 & Basse & $\begin{array}{l}2020-05- \\
19\end{array}$ & France & 141 & Hospital & $\begin{array}{c}62 \\
(52-72)\end{array}$ & 72.0 & 17.7 & - & - & - & - \\
\hline 134 & Freites & $\begin{array}{l}2020-05- \\
19\end{array}$ & Spain & 123 & Hospital & $\begin{array}{l}59.88^{\wedge} \\
(44-74)\end{array}$ & 69.9 & 3.3 & - & - & - & - \\
\hline 135 & Alshami & $\begin{array}{l}2020-05- \\
19\end{array}$ & $\begin{array}{l}\text { Saudi } \\
\text { Arabia }\end{array}$ & 128 & $\begin{array}{l}\text { Quarantine } \\
\text { Centre }\end{array}$ & $\begin{array}{r}39.6^{\wedge} \\
(24-55)\end{array}$ & 53.9 & 15.6 & 2.3 & - & - & - \\
\hline 136 & Berumen & $\begin{array}{l}2020-05- \\
26\end{array}$ & Mexico & 102,875 & Hospital & NA & 49.1 & - & - & 9.6 & - & 90.4 \\
\hline 137 & Gianfrancesco & $\begin{array}{l}2020-05- \\
29\end{array}$ & Multiple & 600 & $\begin{array}{l}\text { Community } \\
\text { and } \\
\text { Hospital }\end{array}$ & $\begin{array}{c}56 \\
(45-67)\end{array}$ & 71.0 & - & - & 21.5 & 64.8 & - \\
\hline 138 & Li, Long & $\begin{array}{l}2020-05- \\
28\end{array}$ & China & 145 & Not Stated & $\begin{array}{c}49^{\wedge} \\
(13-80)\end{array}$ & 61.0 & - & - & 5.5 & - & - \\
\hline 139 & Batty & $\begin{array}{l}2020-06- \\
17\end{array}$ & UK & 908 & Hospital & $\begin{array}{l}57.27^{\wedge} \\
(48-66)\end{array}$ & 44.3 & 11.2 & - & - & - & - \\
\hline 140 & Israel & $\begin{array}{l}2020-06- \\
01\end{array}$ & Israel & 24,906 & $\begin{array}{l}\text { Community } \\
\text { and } \\
\text { Hospital }\end{array}$ & $\begin{array}{c}40 \\
(27-59)\end{array}$ & 48.7 & 16.8 & 12.7 & - & 70.5 & - \\
\hline 141 & del Valle & $\begin{array}{l}2020-05- \\
30\end{array}$ & USA & 1,484 & Hospital & $\begin{array}{c}62 \\
(52-72)\end{array}$ & 40.6 & 5.5 & 23.3 & - & - & - \\
\hline 142 & Chaudhry & $\begin{array}{l}2020-05- \\
29\end{array}$ & USA & 40 & $\begin{array}{l}\text { Community } \\
\text { and } \\
\text { Hospital }\end{array}$ & $\begin{array}{r}52 \\
(45.5- \\
61)\end{array}$ & 60.0 & - & - & 15.0 & - & - \\
\hline 143 & Louis & $\begin{array}{l}2020-05- \\
28\end{array}$ & USA & 22 & Hospital & $\begin{array}{r}66.5^{\wedge} \\
(55-77)\end{array}$ & 36.4 & - & - & 45.5 & - & - \\
\hline 144 & Soto-Mota & $\begin{array}{l}2020-06- \\
05\end{array}$ & Mexico & 400 & Hospital & NA & 30.0 & - & - & 12.0 & - & - \\
\hline 145 & Garibaldi & $\begin{array}{l}2020-05- \\
26\end{array}$ & USA & 832 & Hospital & $\begin{array}{c}63 \\
(49-75)\end{array}$ & 47.0 & 5.5 & 22.6 & - & - & - \\
\hline 146 & Docherty & $\begin{array}{l}2020-05- \\
22\end{array}$ & Multiple & 20,133 & Hospital & $\begin{array}{c}72.9 \\
(58-82)\end{array}$ & 40.0 & 4.2 & 21.7 & - & 44.5 & - \\
\hline \multirow[t]{2}{*}{147} & Boulware & $\begin{array}{l}2020-06- \\
03\end{array}$ & Multiple & 821 & Community & $\begin{array}{c}40 \\
(33-50)\end{array}$ & 51.6 & 3.3 & - & - & - & - \\
\hline & & Sחกח ח & & & Community & ec & & & & & & \\
\hline
\end{tabular}




\begin{tabular}{|c|c|c|c|c|c|c|c|c|c|c|c|c|}
\hline 148 & Kuderer & $\begin{array}{l}\angle U<U-U J^{-} \\
28\end{array}$ & Multiple & 928 & $\begin{array}{l}\text { and } \\
\text { Hospital }\end{array}$ & $\begin{array}{c}\text { סо } \\
(57-76)\end{array}$ & 50.0 & 4.6 & 35.1 & - & 50.5 & - \\
\hline 149 & Romao & $\begin{array}{l}2020-06- \\
08\end{array}$ & Portugal & 34 & Community & $\begin{array}{c}41^{\wedge} \\
(26-66)\end{array}$ & 67.7 & - & - & 26.5 & - & - \\
\hline 150 & Giannouchos & $\begin{array}{l}2020-06- \\
07\end{array}$ & Mexico & 236,439 & $\begin{array}{l}\text { Community } \\
\text { and } \\
\text { Hospital }\end{array}$ & $\begin{array}{r}42.5^{\wedge} \\
(25-59)\end{array}$ & 49.1 & 9.1 & - & - & - & 90.9 \\
\hline 151 & Ramlall & $\begin{array}{l}2020-06- \\
06\end{array}$ & USA & 11,116 & $\begin{array}{l}\text { Community } \\
\text { and } \\
\text { Hospital }\end{array}$ & $\begin{array}{l}52 \\
(34.7- \\
69.5)\end{array}$ & 55.2 & - & - & 26.8 & 73.2 & - \\
\hline 152 & Wang, Oekelen & $\begin{array}{l}2020-06- \\
05\end{array}$ & USA & 58 & $\begin{array}{l}\text { Community } \\
\text { and } \\
\text { Hospital }\end{array}$ & ${ }^{(N A)^{67}}$ & 48.0 & - & - & 36.2 & - & - \\
\hline 153 & Perrone & $\begin{array}{l}2020-06- \\
05\end{array}$ & Italy & 1,189 & Hospital & NA & 21.2 & - & - & 21.9 & - & - \\
\hline 154 & Sharma & $\begin{array}{l}2020-06- \\
05\end{array}$ & India & 501 & Hospital & $\begin{array}{c}35.1^{\wedge} \\
(18-51)\end{array}$ & 36.0 & - & - & 4.2 & - & - \\
\hline 155 & Eugen-Olsen & $\begin{array}{l}2020-06- \\
02\end{array}$ & Denmark & 407 & Hospital & $\begin{array}{c}64 \\
(47-77)\end{array}$ & 57.7 & 20.6 & 36.9 & - & 39.6 & - \\
\hline 156 & $\begin{array}{l}\text { Martinez- } \\
\text { Portilla }\end{array}$ & $\begin{array}{l}2020-06- \\
02\end{array}$ & Mexico & 224 & $\begin{array}{l}\text { Community } \\
\text { and } \\
\text { Hospital }\end{array}$ & $\begin{array}{c}29 \\
(26-33)\end{array}$ & 100.0 & - & - & 3.1 & - & - \\
\hline 157 & $\begin{array}{l}\text { Raisi- } \\
\text { Estabragh }\end{array}$ & $\begin{array}{l}2020-06- \\
02\end{array}$ & UK & 4,510 & Hospital & NA & 48.8 & - & - & 51.8 & - & - \\
\hline 158 & Luo & $\begin{array}{l}2020-06- \\
02\end{array}$ & China & 625 & Hospital & ${ }{ }^{46}{ }^{4}$ & 47.7 & 3.0 & - & - & - & - \\
\hline 159 & Houlihan & $\begin{array}{l}2020-06- \\
09\end{array}$ & UK & 200 & Community & $\begin{array}{c}34 \\
(29-44)\end{array}$ & 61.0 & 11.0 & 16.5 & - & 66.5 & - \\
\hline 160 & Cen & $\begin{array}{l}2020-06- \\
08\end{array}$ & China & 1,007 & Hospital & $\begin{array}{c}61 \\
(49-68)\end{array}$ & 51.0 & - & - & 8.7 & - & - \\
\hline 161 & Klang & $\begin{array}{l}2020-05- \\
23\end{array}$ & USA & 3,406 & Hospital & NA & 61.8 & - & - & 23.3 & - & - \\
\hline 162 & Maraschini & $\begin{array}{l}2020-06- \\
12\end{array}$ & Italy & 146 & Hospital & $\begin{array}{r}32.5^{\wedge} \\
(27-38)\end{array}$ & 100.0 & - & 9.6 & - & 80.8 & - \\
\hline 163 & Wang, Zhong & $\begin{array}{l}2020-06- \\
12\end{array}$ & USA & 7,592 & $\begin{array}{l}\text { Community } \\
\text { and } \\
\text { Hospital }\end{array}$ & NA & 45.1 & 3.6 & 17.1 & - & 51.9 & - \\
\hline 164 & McQueenie & $\begin{array}{l}2020-06- \\
12\end{array}$ & UK & 428,199 & $\begin{array}{l}\text { Community } \\
\text { and } \\
\text { Hospital }\end{array}$ & NA & 54.9 & - & - & 44.4 & 55.0 & - \\
\hline 26 & Miyara & $\begin{array}{l}2020-06- \\
12\end{array}$ & France & 479 & $\begin{array}{l}\text { Community } \\
\text { and } \\
\text { Hospital }\end{array}$ & NA & 44.7 & 6.7 & 31.6 & - & 59.5 & - \\
\hline 165 & Apea & $\begin{array}{l}2020-06- \\
12\end{array}$ & UK & 1,737 & Hospital & $\begin{array}{l}63.4^{\wedge} \\
(\mathrm{NA})\end{array}$ & 30.4 & - & - & 10.0 & - & - \\
\hline 166 & Woolford & $\begin{array}{l}2020-06- \\
11\end{array}$ & UK & 4,510 & $\begin{array}{l}\text { Community } \\
\text { and } \\
\text { Hospital }\end{array}$ & $\begin{array}{l}70.5 \\
\text { (NA) }\end{array}$ & 51.2 & 13.0 & 38.1 & - & 48.1 & - \\
\hline 167 & Hultcrantz & $\begin{array}{l}2020-06- \\
11\end{array}$ & USA & 127 & $\begin{array}{l}\text { Community } \\
\text { and } \\
\text { Hospital }\end{array}$ & $\begin{array}{c}68 \\
(41-91)\end{array}$ & 46.0 & - & - & 26.8 & 72.4 & - \\
\hline 168 & Raiter & 2020-06- & IISA & 280 & Hnsnital & $59.6^{\wedge}$ & 455 & 57 & $1 \cap 7$ & - & 746 & - \\
\hline
\end{tabular}




\begin{tabular}{|c|c|c|c|c|c|c|c|c|c|c|c|c|}
\hline & 'angur & 10 & ひu & $-\cup v$ & murimi & $(41-77)$ & vac & $\cdots$ &... & & , $\cdots$ & \\
\hline 169 & Lan & $\begin{array}{l}2020-06- \\
09\end{array}$ & USA & 104 & Community & $\begin{array}{c}49^{\wedge} \\
(34-63)\end{array}$ & 47.1 & - & - & 24.0 & - & - \\
\hline 170 & Zeng & $\begin{array}{l}2020-06- \\
16\end{array}$ & China & 1,031 & Hospital & $\begin{array}{c}60.3^{\wedge} \\
(46-74)\end{array}$ & 47.8 & - & - & 10.2 & - & - \\
\hline 171 & Suleyman & $\begin{array}{l}2020-06- \\
16\end{array}$ & USA & 463 & Hospital & $\begin{array}{c}57.5^{\wedge} \\
(40-74)\end{array}$ & 55.9 & - & - & 34.6 & - & - \\
\hline 172 & Chen, Yu & $\begin{array}{l}2020-06- \\
16\end{array}$ & China & 1,859 & Hospital & $\begin{array}{c}59 \\
(45-68)\end{array}$ & 50.0 & 2.4 & 3.6 & - & 94.0 & - \\
\hline 173 & Garassino & $\begin{array}{l}2020-06- \\
12\end{array}$ & Multiple & 200 & $\begin{array}{l}\text { Community } \\
\text { and } \\
\text { Hospital }\end{array}$ & $\begin{array}{l}\quad 68 \\
(61.8- \\
75)\end{array}$ & 30.0 & 24.0 & 55.5 & - & 18.5 & - \\
\hline 174 & $\begin{array}{l}\text { Hernandez- } \\
\text { Garduno }\end{array}$ & $\begin{array}{l}2020-06- \\
11\end{array}$ & Mexico & 32,583 & $\begin{array}{l}\text { Community } \\
\text { and } \\
\text { Hospital }\end{array}$ & $\begin{array}{c}45 \\
(34-56)\end{array}$ & 48.7 & - & - & 11.0 & - & 88.8 \\
\hline 175 & Govind & $\begin{array}{l}2020-06- \\
20\end{array}$ & UK & 6,309 & $\begin{array}{l}\text { Community } \\
\text { and } \\
\text { Hospital }\end{array}$ & $\begin{array}{c}46.5^{\wedge} \\
(31-61)\end{array}$ & 38.3 & 66.3 & 26.8 & - & 5.5 & - \\
\hline 176 & Siso-Almirall & $\begin{array}{l}2020-06- \\
20\end{array}$ & Spain & 322 & $\begin{array}{l}\text { Community } \\
\text { and } \\
\text { Hospital }\end{array}$ & $\begin{array}{c}56.7^{\wedge} \\
(38-74)\end{array}$ & 50.0 & - & - & 25.2 & - & - \\
\hline 177 & Gu & $\begin{array}{l}2020-06- \\
18\end{array}$ & USA & 5,698 & $\begin{array}{l}\text { Community } \\
\text { and } \\
\text { Hospital }\end{array}$ & $\begin{array}{c}47^{\wedge} \\
(26-67)\end{array}$ & 62.0 & 7.0 & 24.7 & - & 50.8 & - \\
\hline 178 & Kibler & $\begin{array}{l}2020-06- \\
16\end{array}$ & France & 702 & $\begin{array}{l}\text { Community } \\
\text { and } \\
\text { Hospital }\end{array}$ & $\begin{array}{c}82^{\wedge} \\
(75-88)\end{array}$ & 56.0 & 3.7 & - & - & - & - \\
\hline 179 & Ikitimur & $\begin{array}{l}2020-06- \\
03\end{array}$ & Turkey & 81 & Hospital & $\begin{array}{c}55^{\wedge} \\
(38-72)\end{array}$ & 44.0 & - & - & 28.4 & - & - \\
\hline 180 & Sierpinski & $\begin{array}{l}2020-06- \\
03\end{array}$ & Poland & 1,942 & Community & (NA) $^{50}$ & 60.0 & 6.3 & - & - & - & 49.7 \\
\hline 181 & Zhou, He & $\begin{array}{l}2020-06- \\
10\end{array}$ & China & 238 & Hospital & $\begin{array}{c}55.5 \\
(35-67)\end{array}$ & 57.0 & 2.9 & - & - & - & - \\
\hline 182 & Crovetto & $\begin{array}{l}2020-06- \\
19\end{array}$ & Spain & 874 & $\begin{array}{l}\text { Community } \\
\text { and } \\
\text { Hospital }\end{array}$ & $\begin{array}{r}33.7^{\wedge} \\
(28-38)\end{array}$ & 100.0 & 1.1 & - & - & - & 13.2 \\
\hline 183 & Veras & $\begin{array}{l}2020-06- \\
09\end{array}$ & Brazil & 32 & Hospital & $\begin{array}{c}58.9^{\wedge} \\
(40-77)\end{array}$ & 47.0 & - & - & 25.0 & - & - \\
\hline 184 & Sterlin & $\begin{array}{l}2020-06- \\
11\end{array}$ & France & 135 & Hospital & $\begin{array}{c}61 \\
(50-72)\end{array}$ & 41.0 & 3.7 & 38.5 & - & 57.8 & - \\
\hline 185 & Rossi & $\begin{array}{l}2020-06- \\
09\end{array}$ & France & 246 & Hospital & $\begin{array}{c}68^{\wedge} \\
(53-83)\end{array}$ & 39.0 & - & - & 25.2 & - & - \\
\hline 186 & Duan & $\begin{array}{l}2020-06- \\
22\end{array}$ & China & 616 & Hospital & $\begin{array}{c}64 \\
(53-70)\end{array}$ & 57.5 & 3.7 & - & - & - & - \\
\hline 187 & Martin-Jimenez & $\begin{array}{l}2020-06- \\
09\end{array}$ & Spain & 339 & Hospital & $\begin{array}{c}81.6 \\
(72-87)\end{array}$ & 39.5 & - & - & 30.7 & - & - \\
\hline 188 & Elezkurtaj & $\begin{array}{l}2020-06- \\
17\end{array}$ & Germany & 26 & Hospital & $\begin{array}{l}70 \\
(61.8- \\
78.3)\end{array}$ & 34.6 & - & - & 19.2 & - & - \\
\hline 189 & Lenka & $\begin{array}{l}2020-06- \\
22\end{array}$ & USA & 32 & Hospital & $\begin{array}{c}62.2^{\wedge} \\
(51-73)\end{array}$ & 37.5 & - & - & 50.0 & - & - \\
\hline 190 & Olivares & $\begin{array}{l}2020-06- \\
16\end{array}$ & Chile & 21 & Hospital & $\begin{array}{c}61^{\wedge} \\
(26-85)\end{array}$ & 76.2 & - & - & 9.5 & - & - \\
\hline
\end{tabular}




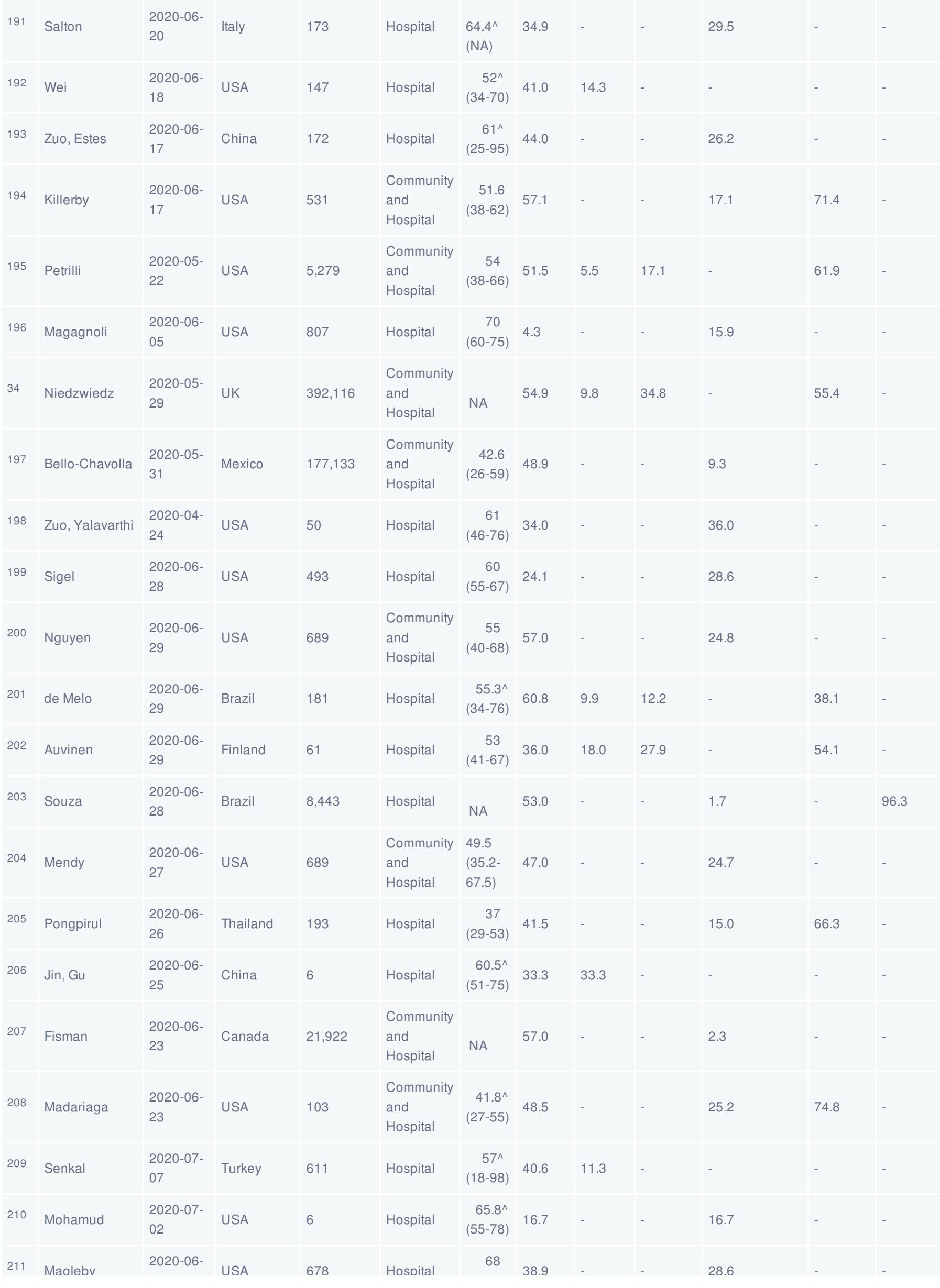




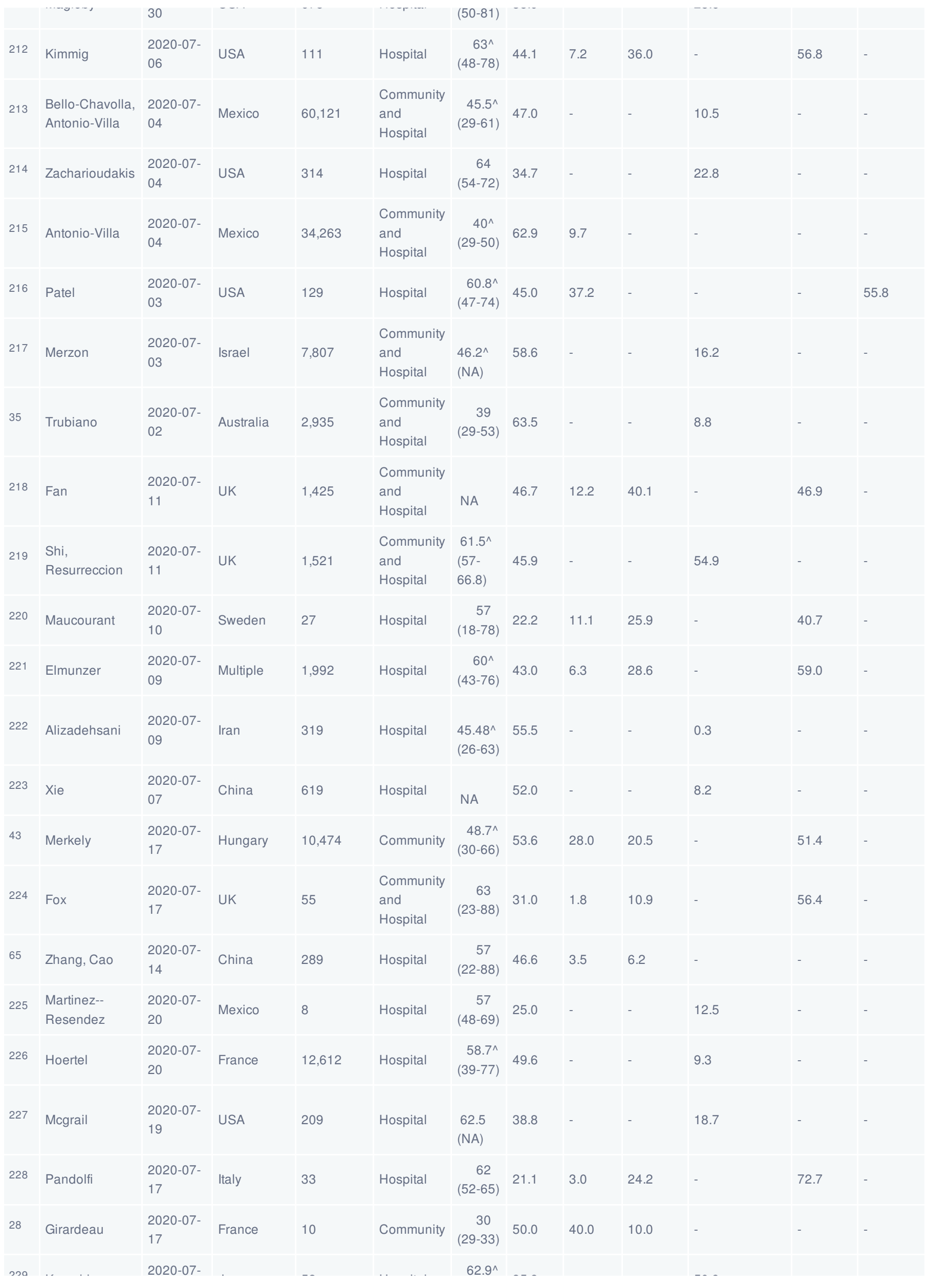




\begin{tabular}{|c|c|c|c|c|c|c|c|c|c|c|c|c|}
\hline$\angle C O$ & Kurashima & 17 & Japan & 53 & Hospital & $(49-76)$ & 35.8 & - & - & 50.9 & - & - \\
\hline 230 & Zhan & $\begin{array}{l}2020-07- \\
16\end{array}$ & China & 75 & Hospital & $\begin{array}{c}57 \\
(25-75)\end{array}$ & 48.0 & - & - & 12.0 & - & - \\
\hline 231 & Omrani & $\begin{array}{l}2020-07- \\
16\end{array}$ & Qatar & 1,409 & $\begin{array}{l}\text { Community } \\
\text { and } \\
\text { Hospital }\end{array}$ & $\begin{array}{c}39 \\
(30-50)\end{array}$ & 17.2 & - & - & 9.2 & - & - \\
\hline 232 & Gupta & $\begin{array}{l}2020-07- \\
16\end{array}$ & USA & 496 & Hospital & $\begin{array}{c}70 \\
(60-78)\end{array}$ & 46.0 & - & - & 7.3 & - & 31.7 \\
\hline 96 & Shi, Zuo & $\begin{array}{l}2020-07- \\
15\end{array}$ & USA & 172 & Hospital & $\begin{array}{l}61.48^{\wedge} \\
(25-96)\end{array}$ & 44.0 & - & - & 26.2 & - & - \\
\hline 233 & Hussein & $\begin{array}{l}2020-07- \\
15\end{array}$ & USA & 502 & Hospital & $\begin{array}{r}60.9^{\wedge} \\
(45-76)\end{array}$ & 52.0 & 9.0 & 22.1 & - & - & 68.9 \\
\hline 234 & Bian & $\begin{array}{l}2020-07- \\
15\end{array}$ & China & 28 & Hospital & $\begin{array}{c}56^{\wedge} \\
(42-67)\end{array}$ & 42.9 & 7.1 & - & - & - & - \\
\hline 235 & Eiros & $\begin{array}{l}2020-07- \\
14\end{array}$ & Spain & 139 & $\begin{array}{l}\text { Community } \\
\text { and } \\
\text { Hospital }\end{array}$ & $\begin{array}{c}52 \\
(41-57)\end{array}$ & 72.0 & 4.3 & 50.4 & - & - & - \\
\hline 236 & Marcos & $\begin{array}{l}2020-07- \\
14\end{array}$ & Spain & 918 & Hospital & $\begin{array}{c}72.8^{\wedge} \\
(58-87)\end{array}$ & 42.2 & 6.1 & - & 15.3 & - & - \\
\hline 237 & $\begin{array}{l}\text { Hoertel, } \\
\text { Sanchez-Rico }\end{array}$ & $\begin{array}{l}2020-07- \\
14\end{array}$ & France & 7,345 & Hospital & NA & 49.3 & 8.5 & - & - & - & - \\
\hline 238 & Soares & $\begin{array}{l}2020-07- \\
16\end{array}$ & Brazil & 10,713 & $\begin{array}{l}\text { Community } \\
\text { and } \\
\text { Hospital }\end{array}$ & NA & 55.0 & 2.0 & - & - & - & 98.0 \\
\hline 239 & Zobairy & $\begin{array}{l}2020-07- \\
28\end{array}$ & Iran & 203 & $\begin{array}{l}\text { Community } \\
\text { and } \\
\text { Hospital }\end{array}$ & $\begin{array}{r}49.2^{\wedge} \\
(32-65)\end{array}$ & 44.8 & 5.9 & - & - & - & 94.1 \\
\hline 240 & Altamimi & $\begin{array}{l}2020-07- \\
27\end{array}$ & Qatar & 68 & Hospital & $\begin{array}{c}49^{\wedge} \\
(40-58)\end{array}$ & 2.0 & 16.4 & - & - & - & 83.6 \\
\hline 241 & Thompson & $\begin{array}{l}2020-07- \\
27\end{array}$ & UK & 470 & Hospital & $\begin{array}{c}71 \\
(57-82)\end{array}$ & 46.0 & 14.0 & 27.2 & - & 58.7 & - \\
\hline 242 & Reiter & $\begin{array}{l}2020-07- \\
26\end{array}$ & Austria & 235 & Community & $\begin{array}{r}44.2^{\wedge} \\
(32-55)\end{array}$ & 70.0 & 22.6 & 22.6 & - & 54.7 & - \\
\hline 243 & Motta & $\begin{array}{l}2020-07- \\
26\end{array}$ & USA & 374 & Hospital & $\begin{array}{r}64.7^{\wedge} \\
(46-82)\end{array}$ & 41.4 & - & - & 33.2 & 66.8 & - \\
\hline 244 & Santos & $\begin{array}{l}2020-07- \\
25\end{array}$ & USA & 43 & $\begin{array}{l}\text { Community } \\
\text { and } \\
\text { Hospital }\end{array}$ & $\begin{array}{c}50 \\
(34-73)\end{array}$ & 63.0 & - & - & 4.7 & - & - \\
\hline 245 & Schneeweiss & $\begin{array}{l}2020-07- \\
22\end{array}$ & USA & 24,313 & $\begin{array}{l}\text { Community } \\
\text { and } \\
\text { Hospital }\end{array}$ & $\begin{array}{c}67^{\wedge} \\
(53-80)\end{array}$ & 53.0 & - & - & 2.9 & - & - \\
\hline 246 & Concha-Mejia & $\begin{array}{l}2020-07- \\
24\end{array}$ & Colombia & 72 & $\begin{array}{l}\text { Community } \\
\text { and } \\
\text { Hospital }\end{array}$ & $\begin{array}{c}46 \\
(28-64)\end{array}$ & 47.0 & 8.3 & 11.1 & - & - & - \\
\hline 247 & Izquierdo & $\begin{array}{l}2020-07- \\
24\end{array}$ & Spain & 71,192 & $\begin{array}{l}\text { Community } \\
\text { and } \\
\text { Hospital }\end{array}$ & $\begin{array}{c}42^{\wedge} \\
(18-66)\end{array}$ & 59.0 & 10.0 & - & - & - & 90.0 \\
\hline 248 & Bernaola & $\begin{array}{l}2020-07- \\
21\end{array}$ & Spain & 1,645 & Hospital & NA & 38.5 & 2.5 & 10.9 & - & 86.6 & - \\
\hline 30 & Islam & $\begin{array}{l}2020-08- \\
18\end{array}$ & Bangladesh & 1,016 & $\begin{array}{l}\text { Community } \\
\text { and } \\
\text { Hospital }\end{array}$ & $\begin{array}{c}37 \\
(28-49)\end{array}$ & 35.9 & 18.2 & - & - & - & - \\
\hline
\end{tabular}




\begin{tabular}{|c|c|c|c|c|c|c|c|c|c|c|c|c|}
\hline 249 & Qi & $\begin{array}{l}2020-03- \\
03\end{array}$ & China & 267 & Hospital & $\begin{array}{c}48 \\
(35-65)\end{array}$ & 45.2 & 19.9 & - & - & - & 80.1 \\
\hline 250 & Peters & $\begin{array}{l}2020-08- \\
15\end{array}$ & Netherlands & 1,893 & Hospital & $\begin{array}{c}66.8^{\wedge} \\
(52-81)\end{array}$ & 39.4 & 4.9 & - & - & - & - \\
\hline 251 & Ouyang & $\begin{array}{l}2020-08- \\
14\end{array}$ & China & 217 & Hospital & $\begin{array}{r}46.5^{\wedge} \\
(30-62)\end{array}$ & 53.5 & 16.6 & - & - & - & - \\
\hline 56 & Ward & $\begin{array}{l}2020-08- \\
21\end{array}$ & UK & 99,908 & Community & NA & 56.1 & 10.6 & - & - & - & 88.4 \\
\hline 252 & Valenzuela & $\begin{array}{l}2020-08- \\
14\end{array}$ & Chile & 29 & Hospital & $\begin{array}{c}56.9^{\wedge} \\
(43-70)\end{array}$ & 6.9 & 17.2 & - & - & - & 82.8 \\
\hline 253 & Monteiro & $\begin{array}{l}2020-08- \\
14\end{array}$ & USA & 112 & Hospital & $\begin{array}{c}61 \\
(45-74)\end{array}$ & 34.0 & 6.2 & 17.9 & - & 68.8 & - \\
\hline 254 & Philipose & $\begin{array}{l}2020-08- \\
14\end{array}$ & UK & 466 & Hospital & $\begin{array}{r}67 \\
(6-97)\end{array}$ & 41.8 & 6.0 & 73.2 & - & 16.5 & - \\
\hline 255 & Weerahandi & $\begin{array}{l}2020-08- \\
14\end{array}$ & USA & 394 & Community & $\begin{array}{c}63 \\
(55-70)\end{array}$ & 37.0 & 5.3 & 25.9 & - & 55.8 & - \\
\hline 29 & Ebinger & $\begin{array}{l}2020-08- \\
04\end{array}$ & USA & 6,062 & Community & $\begin{array}{c}41.5^{\wedge} \\
(29-53)\end{array}$ & 67.8 & 1.7 & - & - & - & - \\
\hline 256 & Altibi & $\begin{array}{l}2020-08- \\
11\end{array}$ & USA & 706 & Hospital & $\begin{array}{c}66.7^{\wedge} \\
(51-81)\end{array}$ & 43.0 & 4.0 & 37.3 & - & 58.8 & - \\
\hline 257 & Izzi-Engbeaya & $\begin{array}{l}2020-08- \\
11\end{array}$ & UK & 889 & Hospital & $\begin{array}{r}65.8^{\wedge} \\
(48-83)\end{array}$ & 40.0 & - & - & 21.3 & 33.2 & - \\
\hline 258 & Rizzo & $\begin{array}{l}2020-08- \\
11\end{array}$ & USA & 76,819 & Hospital & $\begin{array}{c}54 \\
(38-67)\end{array}$ & 55.2 & 6.7 & 20.8 & - & 50.4 & - \\
\hline 259 & Dashti & $\begin{array}{l}2020-08- \\
04\end{array}$ & USA & 4,140 & $\begin{array}{l}\text { Community } \\
\text { and } \\
\text { Hospital }\end{array}$ & $\begin{array}{c}52 \\
(36-65)\end{array}$ & 55.0 & - & - & 28.4 & 51.6 & - \\
\hline 260 & Morshed & $\begin{array}{l}2020-08- \\
02\end{array}$ & Bangladesh & 103 & Community & $\begin{array}{c}37 \\
(31-53)\end{array}$ & 28.2 & 31.1 & - & - & - & 68.9 \\
\hline 261 & Jun & $\begin{array}{l}2020-08- \\
01\end{array}$ & USA & 3,086 & Hospital & $\begin{array}{c}66 \\
(56-77)\end{array}$ & 40.9 & 3.7 & 21.3 & - & 52.8 & - \\
\hline 262 & Higuchi & $\begin{array}{l}2020-07- \\
30\end{array}$ & Japan & 57 & Hospital & $\begin{array}{c}52 \\
(35-70)\end{array}$ & 43.9 & 12.3 & 29.8 & - & 57.9 & - \\
\hline 263 & Zhou, Sun & $\begin{array}{l}2020-07- \\
29\end{array}$ & China & 144 & Hospital & $\begin{array}{c}47 \\
(38-56)\end{array}$ & 46.5 & 9.0 & - & - & - & 91.0 \\
\hline 264 & Salerno & $\begin{array}{l}2020-08- \\
22\end{array}$ & USA & 15,920 & Hospital & $\begin{array}{c}49 \\
(30-65)\end{array}$ & 57.0 & - & - & 36.8 & 55.9 & - \\
\hline 265 & Kumar & $\begin{array}{l}2020-07- \\
29\end{array}$ & India & 91 & Hospital & $\begin{array}{c}47^{\wedge} \\
(41-52)\end{array}$ & 21.0 & 44.0 & - & - & - & - \\
\hline 266 & $\mathrm{HaO}$ & $\begin{array}{l}2020-06- \\
01\end{array}$ & China & 788 & Hospital & $\begin{array}{c}46 \\
(35-56)\end{array}$ & 48.4 & 6.9 & - & - & - & - \\
\hline 267 & Iversen & $\begin{array}{l}2020-08- \\
03\end{array}$ & Denmark & 28,792 & $\begin{array}{l}\text { Community } \\
\text { and } \\
\text { Hospital }\end{array}$ & $\begin{array}{r}44.4^{\wedge} \\
(31-57)\end{array}$ & 78.9 & 16.0 & 6.5 & - & 76.8 & - \\
\hline 268 & Hippisley-Cox & $\begin{array}{l}2020-07- \\
13\end{array}$ & UK & $8,275,949$ & $\begin{array}{l}\text { Community } \\
\text { and } \\
\text { Hospital }\end{array}$ & $\begin{array}{r}48.5^{\wedge} \\
(30-66)\end{array}$ & 50.3 & 17.2 & 21.4 & - & 57.3 & - \\
\hline 269 & Fillmore & $\begin{array}{l}2020-08- \\
24\end{array}$ & USA & 22,914 & $\begin{array}{l}\text { Community } \\
\text { and } \\
\text { Hospital }\end{array}$ & NA & - & 37.5 & 40.7 & - & 15.5 & - \\
\hline 270 & Rashid & 2020-08- & UK & 517 & Hospital & $72.8^{\wedge}$ & 31.9 & 9.9 & 29.0 & - & 29.4 & - \\
\hline
\end{tabular}




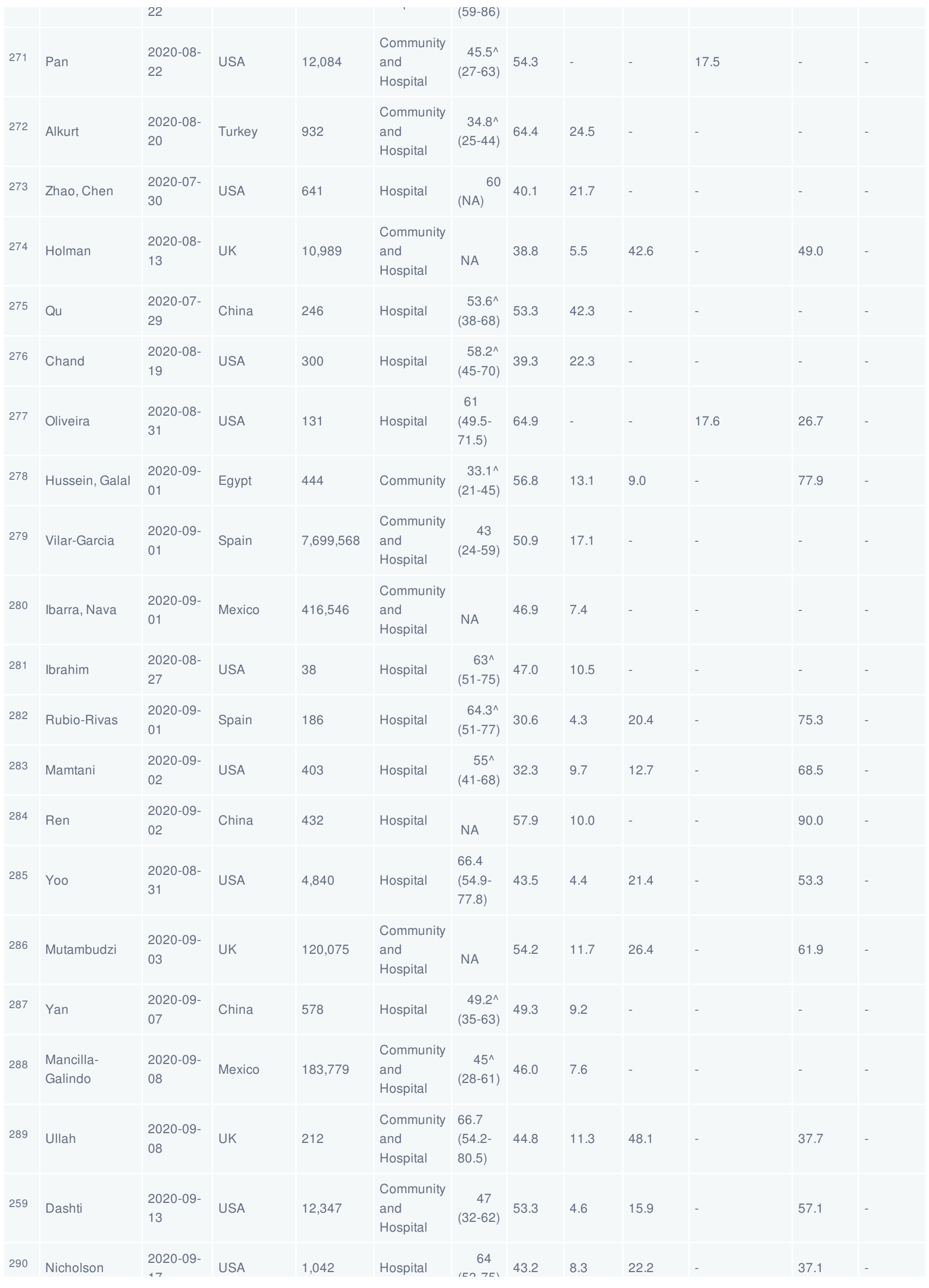




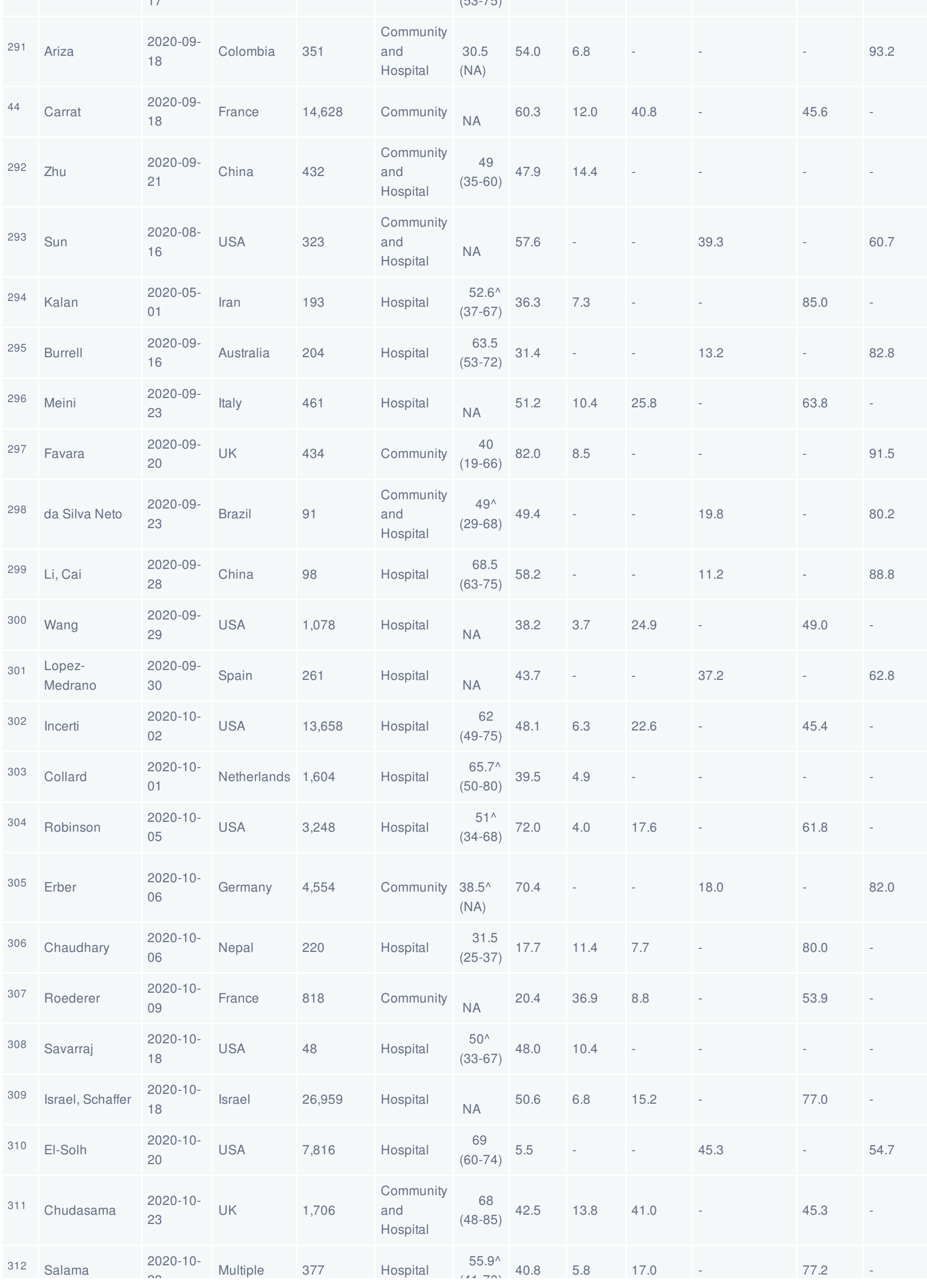




\begin{tabular}{|c|c|c|c|c|c|c|c|c|c|c|c|c|}
\hline & & 23 & & & & $(41-/ U)$ & & & & & & \\
\hline 313 & Makaronidis & $\begin{array}{l}2020-10- \\
01\end{array}$ & UK & 567 & Community & $\begin{array}{r}39.4^{\wedge} \\
(27-51)\end{array}$ & 69.1 & 9.3 & - & - & - & 90.7 \\
\hline 314 & Ramachandran & $\begin{array}{l}2020-10- \\
12\end{array}$ & USA & 188 & Hospital & NA & - & 18.6 & - & - & - & - \\
\hline 315 & Hadi & $\begin{array}{l}2020-08- \\
05\end{array}$ & USA & 370 & $\begin{array}{l}\text { Community } \\
\text { and } \\
\text { Hospital }\end{array}$ & $\begin{array}{r}48.2^{\wedge} \\
(34-62)\end{array}$ & 29.5 & - & - & 15.1 & 84.9 & - \\
\hline 316 & Luo, Rizvi & $\begin{array}{l}2020-10- \\
03\end{array}$ & USA & 102 & Hospital & $\begin{array}{c}68 \\
(61-75)\end{array}$ & 52.0 & - & - & 26.5 & - & 73.5 \\
\hline 317 & loannou & $\begin{array}{l}2020-09- \\
23\end{array}$ & USA & 88,747 & $\begin{array}{l}\text { Community } \\
\text { and } \\
\text { Hospital }\end{array}$ & NA & 9.0 & 20.6 & 37.5 & - & 29.3 & - \\
\hline 318 & ISARIC & $\begin{array}{l}2020-10- \\
04\end{array}$ & Multiple & 88,463 & Hospital & $(\mathrm{NA})^{72}$ & 43.0 & - & - & 5.2 & - & 37.6 \\
\hline 319 & Perico & $\begin{array}{l}2020-10- \\
22\end{array}$ & Italy & 423 & Community & $\begin{array}{r}44.3^{\wedge} \\
(34-54)\end{array}$ & 36.4 & 21.7 & 18.0 & - & 60.3 & - \\
\hline 320 & Lamure & $\begin{array}{l}2020-10- \\
12\end{array}$ & France & 89 & Hospital & $\begin{array}{c}67 \\
(19-92)\end{array}$ & 34.0 & 5.6 & 32.6 & - & 48.3 & - \\
\hline 321 & Yadaw & $\begin{array}{l}2020-10- \\
01\end{array}$ & USA & 5,051 & $\begin{array}{l}\text { Community } \\
\text { and } \\
\text { Hospital }\end{array}$ & NA & - & 3.6 & 15.9 & - & 51.4 & - \\
\hline 322 & Zinellu & $\begin{array}{l}2020-10- \\
11\end{array}$ & Italy & 105 & Hospital & $\begin{array}{l}72 \\
(59.5- \\
80)\end{array}$ & 33.3 & 30.5 & 10.5 & - & 59.0 & - \\
\hline 323 & Ziehr & $\begin{array}{l}2020-04- \\
29\end{array}$ & USA & 66 & Hospital & $\begin{array}{l}58(23- \\
87)\end{array}$ & 35.0 & - & - & 33.3 & 63.6 & - \\
\hline 324 & Zhou, He, Yang & $\begin{array}{l}2020-10- \\
27\end{array}$ & China & 1,087 & Hospital & NA & 51.7 & - & - & 85.0 & 15.0 & - \\
\hline 325 & Zhou, Song & $\begin{array}{l}2020-10- \\
22\end{array}$ & China & 124 & Hospital & $\begin{array}{l}67(30- \\
86)\end{array}$ & 48.0 & 19.1 & - & - & - & - \\
\hline 326 & Zhou, Qin & $\begin{array}{l}2020-08- \\
25\end{array}$ & China & 51 & Hospital & $\begin{array}{l}57.37^{\wedge} \\
(42-72)\end{array}$ & 29.4 & - & - & 78.4 & 21.6 & - \\
\hline 327 & Zhang, Li & $\begin{array}{l}2020-12- \\
06\end{array}$ & UK & 1,746 & $\begin{array}{l}\text { Community } \\
\text { and } \\
\text { Hospital }\end{array}$ & $\begin{array}{l}68.8^{\wedge} \\
(59-78)\end{array}$ & 47.1 & 10.1 & 35.1 & - & 44.2 & - \\
\hline 328 & Zhan, Liu & $\begin{array}{l}2020-08- \\
31\end{array}$ & China & 405 & Hospital & $\begin{array}{l}56^{\wedge} \\
(17-95)\end{array}$ & 54.1 & - & - & 11.4 & 88.6 & - \\
\hline 329 & Wang, Shu & $\begin{array}{l}2020-07- \\
20\end{array}$ & China & 59 & Hospital & $\begin{array}{l}67.4^{\wedge} \\
(56-78)\end{array}$ & 35.6 & - & - & 15.3 & - & - \\
\hline 330 & Wang, Zheutlin & $\begin{array}{l}2020-10- \\
26\end{array}$ & USA & 3,273 & Hospital & $\begin{array}{l}65 \text { (53- } \\
77)\end{array}$ & 42.7 & 3.5 & 20.7 & - & 53.2 & - \\
\hline 331 & Vila-Corcoles & $\begin{array}{l}2020-12- \\
10\end{array}$ & Spain & 79,083 & Community & NA & 52.4 & - & - & 16.1 & - & - \\
\hline 332 & Torres-Macho & $\begin{array}{l}2020-09- \\
23\end{array}$ & Spain & 1,968 & Hospital & NA & 44.0 & - & - & 23.4 & - & - \\
\hline 333 & Tao & $\begin{array}{l}2020-08- \\
06\end{array}$ & China & 70 & Hospital & $\begin{array}{l}33.24^{\wedge} \\
(12-53)\end{array}$ & 48.6 & - & - & 15.7 & - & - \\
\hline 334 & Talavera & $\begin{array}{l}2020-10- \\
01\end{array}$ & Spain & 576 & Hospital & $\begin{array}{l}67.2^{\wedge} \\
(52-81)\end{array}$ & 43.4 & - & - & 20.5 & - & - \\
\hline 335 & Serling-Boyd & $\begin{array}{l}2020-11- \\
30\end{array}$ & USA & 831 & Hospital & NA & 76.0 & 3.0 & 23.2 & - & 50.1 & - \\
\hline
\end{tabular}




\begin{tabular}{|c|c|c|c|c|c|c|c|c|c|c|c|c|}
\hline 336 & Raines & $\begin{array}{l}2020-10- \\
07\end{array}$ & USA & 453 & $\begin{array}{l}\text { Community } \\
\text { and } \\
\text { Hospital }\end{array}$ & $\begin{array}{l}60.8^{\wedge} \\
(46-74)\end{array}$ & 10.7 & - & - & 53.0 & 41.9 & - \\
\hline 337 & $\begin{array}{l}\text { Parra- } \\
\text { Bracamonte }\end{array}$ & $\begin{array}{l}2020-08- \\
14\end{array}$ & Mexico & 331,298 & $\begin{array}{l}\text { Community } \\
\text { and } \\
\text { Hospital }\end{array}$ & $\begin{array}{l}44(33- \\
56)\end{array}$ & 46.2 & - & - & 7.4 & - & - \\
\hline 338 & O'Reilly & $\begin{array}{l}2020-09- \\
21\end{array}$ & Australia & 1,334 & Hospital & NA & - & - & - & 28.5 & - & - \\
\hline 339 & Martini & $\begin{array}{l}2020-12- \\
04\end{array}$ & Italy & 146 & Hospital & NA & 49.0 & - & - & 46.6 & 53.4 & - \\
\hline 340 & Li, Long, Zhang & $\begin{array}{l}2020-12- \\
03\end{array}$ & China & 954 & Hospital & NA & - & - & - & 5.9 & 94.1 & - \\
\hline 341 & Lassale & $\begin{array}{l}2020-06- \\
01\end{array}$ & UK & 900 & Hospital & $\begin{array}{l}57.2^{\wedge} \\
(48-66)\end{array}$ & 44.4 & 11.4 & 41.9 & - & 46.7 & - \\
\hline 342 & Klang, Soffer & $\begin{array}{l}2020-08- \\
09\end{array}$ & USA & 1,320 & Hospital & NA & 41.5 & - & - & 24.7 & - & - \\
\hline 343 & Kim, Han & $\begin{array}{l}2020-11- \\
09\end{array}$ & $\begin{array}{l}\text { South } \\
\text { Korea }\end{array}$ & 4,787 & Hospital & $\begin{array}{l}55(38- \\
68)\end{array}$ & 60.1 & 5.5 & 2.8 & - & 91.7 & - \\
\hline 344 & Jehi & $\begin{array}{l}2020-08- \\
11\end{array}$ & USA & 4,536 & $\begin{array}{l}\text { Community } \\
\text { and } \\
\text { Hospital }\end{array}$ & NA & - & 7.3 & 28.5 & - & 49.9 & - \\
\hline 345 & Jakob & $\begin{array}{l}2020-10- \\
01\end{array}$ & Multiple & 2,155 & $\begin{array}{l}\text { Community } \\
\text { and } \\
\text { Hospital }\end{array}$ & NA & 40.3 & 6.6 & 7.3 & - & 34.3 & - \\
\hline 346 & Invernizzi & $\begin{array}{l}2020-09- \\
20\end{array}$ & Italy & 54 & Hospital & $\begin{array}{l}49.9^{\wedge} \\
(34-65)\end{array}$ & 29.7 & - & - & 24.1 & - & - \\
\hline 347 & Ilic & $\begin{array}{l}2020-11- \\
19\end{array}$ & Serbia & 107 & $\begin{array}{l}\text { Community } \\
\text { and } \\
\text { Hospital }\end{array}$ & $\begin{array}{l}39.1^{\wedge} \\
(27-50)\end{array}$ & - & 29.9 & - & - & - & - \\
\hline 348 & Hamadah & $\begin{array}{l}2020-09- \\
10\end{array}$ & Kuwait & 1,123 & Hospital & $\begin{array}{l}40(1- \\
93)\end{array}$ & 18.7 & 3.9 & - & - & - & 96.1 \\
\hline 349 & $\begin{array}{l}\text { Gianfrancesco, } \\
\text { Leykina }\end{array}$ & $\begin{array}{l}2020-11- \\
03\end{array}$ & USA & 1,324 & $\begin{array}{l}\text { Community } \\
\text { and } \\
\text { Hospital }\end{array}$ & NA & 75.9 & - & - & 26.7 & 68.2 & - \\
\hline 350 & Ghinai & $\begin{array}{l}2020-10- \\
12\end{array}$ & USA & 1,435 & $\begin{array}{l}\text { Homeless } \\
\text { Shelters }\end{array}$ & NA & 27.6 & 36.6 & 17.5 & - & 33.2 & - \\
\hline 351 & Fond & $\begin{array}{l}2020-07- \\
30\end{array}$ & France & 1,092 & Hospital & $\begin{array}{l}62.5 \\
(51-76)\end{array}$ & 45.7 & 11.4 & - & - & - & 88.6 \\
\hline 352 & Best & $\begin{array}{l}2020-10- \\
12\end{array}$ & USA & 3,471 & Hospital & $\begin{array}{l}63.5^{\wedge} \\
(47-79)\end{array}$ & 51.2 & - & - & 28.6 & - & 71.4 \\
\hline 353 & Bellan & $\begin{array}{l}2020-11- \\
26\end{array}$ & Italy & 1,697 & Hospital & $\begin{array}{l}71(58- \\
80)\end{array}$ & 41.0 & 3.2 & - & - & - & 15.2 \\
\hline 354 & Alharthy & $\begin{array}{l}2020-10- \\
03\end{array}$ & $\begin{array}{l}\text { Saudi } \\
\text { Arabia }\end{array}$ & 352 & Hospital & $\begin{array}{l}50.6^{\wedge} \\
(37-63)\end{array}$ & 12.8 & 49.4 & - & - & - & 50.6 \\
\hline 355 & Alguwaihes & $\begin{array}{l}2020-12- \\
05\end{array}$ & $\begin{array}{l}\text { Saudi } \\
\text { Arabia }\end{array}$ & 439 & Hospital & $\begin{array}{l}55(19- \\
101)\end{array}$ & 31.7 & 2.1 & - & - & - & 97.9 \\
\hline 356 & Aksu & $\begin{array}{l}2020-08- \\
19\end{array}$ & Turkey & 123 & $\begin{array}{l}\text { Community } \\
\text { and } \\
\text { Hospital }\end{array}$ & $\begin{array}{l}49.7^{\wedge} \\
(36-63)\end{array}$ & 33.3 & 11.4 & - & - & - & 88.6 \\
\hline 357 & Adrish & $\begin{array}{l}2020-10- \\
05\end{array}$ & USA & 1,173 & Hospital & NA & 38.6 & 14.0 & 14.7 & - & 71.4 & - \\
\hline 258 & Hoertel, & 2020-10- & - & & . & & & $\ldots$ & & & & \\
\hline
\end{tabular}




\begin{tabular}{|c|c|c|c|c|c|c|c|c|c|c|c|c|}
\hline unu & $\begin{array}{l}\text { Sanchez, } \\
\text { Vernet }\end{array}$ & 27 & France & 12,210 & Hospital & NA & 50.1 & 9.0 & - & - & - & - \\
\hline 359 & Arleo & $\begin{array}{l}2020-10- \\
27\end{array}$ & USA & 70 & $\begin{array}{l}\text { Community } \\
\text { and } \\
\text { Hospital }\end{array}$ & $\begin{array}{l}56.6^{\wedge} \\
(48-65)\end{array}$ & 80.0 & 1.4 & 28.6 & - & 70.0 & - \\
\hline 360 & $\begin{array}{l}\text { Bermejo, } \\
\text { Martin }\end{array}$ & $\begin{array}{l}2020-10- \\
27\end{array}$ & Spain & 250 & $\begin{array}{l}\text { Community } \\
\text { and } \\
\text { Hospital }\end{array}$ & NA & 64.0 & 6.0 & - & - & - & - \\
\hline 361 & Joubert & $\begin{array}{l}2020-10- \\
29\end{array}$ & France & 74 & $\begin{array}{l}\text { Community } \\
\text { and } \\
\text { Hospital }\end{array}$ & NA & - & 5.4 & - & - & - & - \\
\hline 362 & Kortela & $\begin{array}{l}2020-11- \\
01\end{array}$ & Finland & 3,008 & $\begin{array}{l}\text { Community } \\
\text { and } \\
\text { Hospital }\end{array}$ & $\begin{array}{l}51(36- \\
69)\end{array}$ & 59.6 & 11.0 & 14.0 & - & 29.2 & - \\
\hline 363 & Sourij & $\begin{array}{l}2020-11- \\
16\end{array}$ & Austria & 238 & Hospital & $\begin{array}{l}71.1^{\wedge} \\
(58-83)\end{array}$ & 36.1 & 1.7 & 16.0 & - & 82.4 & - \\
\hline 31 & Gallichotte & $\begin{array}{l}2020-11- \\
05\end{array}$ & USA & 239 & Community & $\begin{array}{l}41^{\wedge} \\
(16-76)\end{array}$ & - & 20.1 & 16.7 & - & 57.3 & - \\
\hline 364 & Galal & $\begin{array}{l}2020-11- \\
12\end{array}$ & Egypt & 430 & Community & $\begin{array}{l}37.4^{\wedge} \\
(24-50)\end{array}$ & 63.7 & 6.0 & 7.7 & - & 86.3 & - \\
\hline 365 & Clavario & $\begin{array}{l}2020-11- \\
16\end{array}$ & Italy & 110 & Community & $\begin{array}{l}61.7 \\
(53.5- \\
69.2)\end{array}$ & 40.9 & 40.9 & - & - & - & - \\
\hline 366 & Saeed & $\begin{array}{l}2020-11- \\
16\end{array}$ & $\begin{array}{l}\text { United Arab } \\
\text { Emirates }\end{array}$ & 173 & Hospital & NA & 34.1 & 6.4 & - & - & - & - \\
\hline 367 & Cadegiani & $\begin{array}{l}2020-11- \\
18\end{array}$ & Brazil & 130 & Community & $\begin{array}{l}42^{\wedge} \\
(\mathrm{NA})\end{array}$ & 0.0 & 7.7 & - & - & - & - \\
\hline 368 & Benaim & $\begin{array}{l}2020-11- \\
19\end{array}$ & Israel & 693 & Hospital & $\begin{array}{l}59.8 \\
(\mathrm{NA})\end{array}$ & 47.9 & - & - & 5.1 & - & - \\
\hline 369 & Singh & $\begin{array}{l}2020-11- \\
20\end{array}$ & UK & 930 & Hospital & $\begin{array}{l}71.4^{\wedge} \\
(54-87)\end{array}$ & 44.8 & - & - & 19.0 & - & 81.0 \\
\hline 370 & ISARIC & $\begin{array}{l}2020-11- \\
23\end{array}$ & Multiple & 95,966 & Hospital & $\begin{array}{l}72 \\
\text { (NA) }\end{array}$ & 43.0 & 5.2 & - & - & - & 37.5 \\
\hline 371 & $\begin{array}{l}\text { Marquez- } \\
\text { Salinas }\end{array}$ & $\begin{array}{l}2020-11- \\
24\end{array}$ & Mexico & 1,068 & Hospital & $\begin{array}{l}53(44- \\
63)\end{array}$ & 36.8 & - & - & 15.0 & - & - \\
\hline 372 & Diez-Manglano & $\begin{array}{l}2020-11- \\
24\end{array}$ & Spain & 4,393 & Hospital & $\begin{array}{l}53^{\wedge} \\
(\mathrm{NA})\end{array}$ & 40.8 & 6.6 & - & - & - & - \\
\hline 373 & Woolcott & $\begin{array}{l}2020-11- \\
26\end{array}$ & Mexico & $1,636,050$ & $\begin{array}{l}\text { Community } \\
\text { and } \\
\text { Hospital }\end{array}$ & $\begin{array}{l}42(34- \\
54)\end{array}$ & 51.9 & - & - & 8.8 & - & - \\
\hline 374 & Simons & $\begin{array}{l}2020-11- \\
30\end{array}$ & UK & 446 & Hospital & $\begin{array}{l}64.9 \\
(52.4- \\
76.2)\end{array}$ & 35.9 & 9.4 & 38.6 & - & 52.0 & - \\
\hline 375 & Dupraz & $\begin{array}{l}2020-11- \\
30\end{array}$ & Switzerland & 219 & Community & NA & 54.8 & 11.0 & - & - & - & - \\
\hline 376 & $\begin{array}{l}\text { Chen, } \\
\text { Varathraja }\end{array}$ & $\begin{array}{l}2020-11- \\
29\end{array}$ & USA & 10,123 & $\begin{array}{l}\text { Community } \\
\text { and } \\
\text { Hospital }\end{array}$ & $\begin{array}{l}40(28- \\
54)\end{array}$ & 53.6 & 4.4 & 9.7 & - & 45.9 & - \\
\hline 377 & $\begin{array}{l}\text { Martinez, } \\
\text { Lacalzada }\end{array}$ & $\begin{array}{l}2020-12- \\
01\end{array}$ & Spain & 10,433 & Hospital & $\begin{array}{l}65.8^{\wedge} \\
(49-82)\end{array}$ & 42.8 & 5.2 & 23.8 & - & 66.0 & - \\
\hline 378 & Barasa & $\begin{array}{l}2020-11- \\
30\end{array}$ & USA & 394 & Hospital & NA & 47.7 & 14.7 & 36.0 & - & 41.6 & - \\
\hline 79 & & 2020-11- & & & & $n$ & $A$ & $\neg-$ & 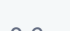 & & & \\
\hline
\end{tabular}




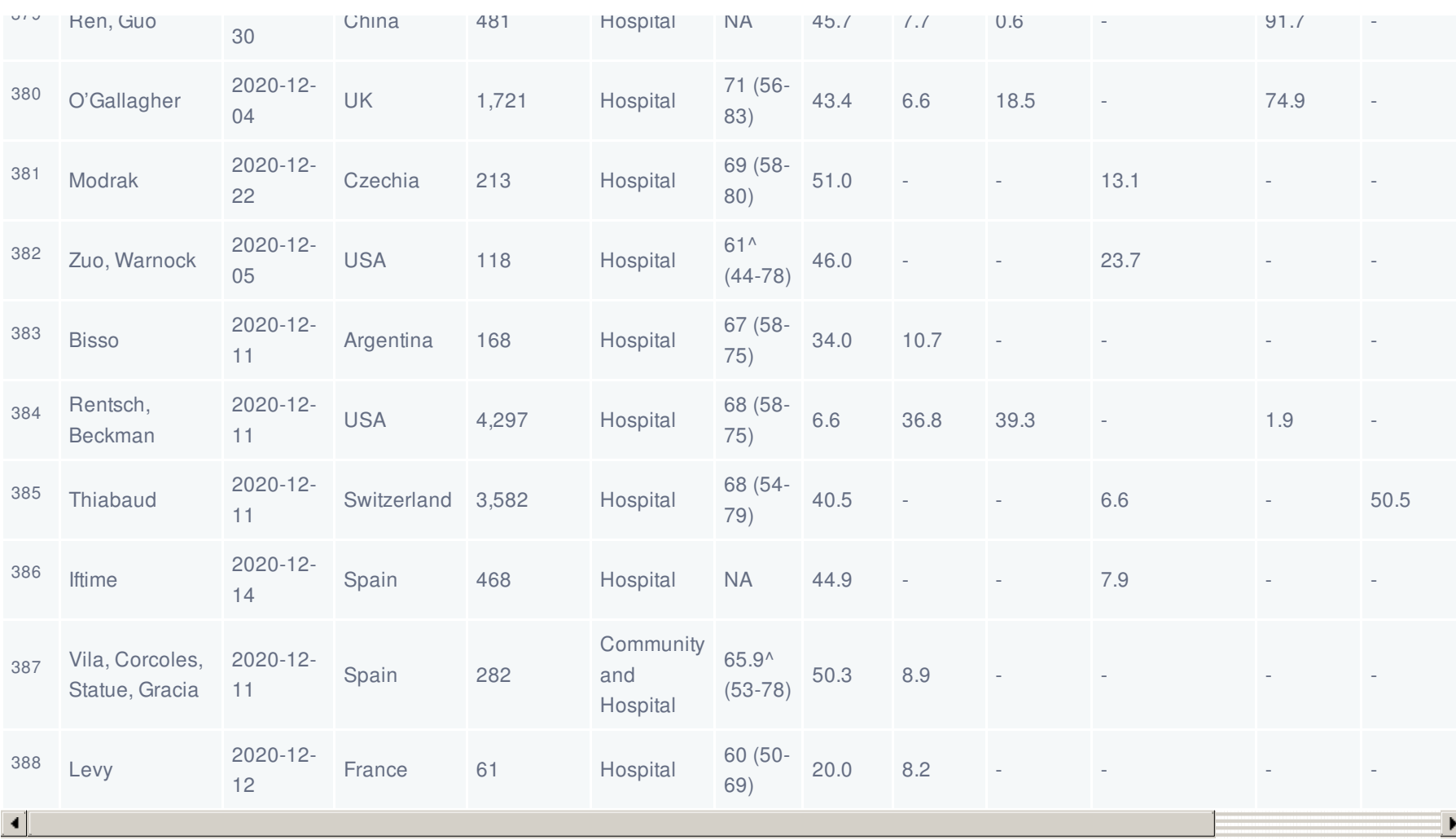

Note. - Age not provided for total sample; ^ Denotes mean (SD). * This study was rated as 'poor' quality as the manuscript only presents data for current (but not former) smokers despite having obtained complete smoking status, thus resulting in $>20 \%$ missing data on smoking status.

\section{Smoking prevalence by country}

Unadjusted smoking prevalence compared with overall estimates for national adult smoking prevalence split by country and study setting is presented in Figure $2 \mathrm{a}$ and $2 \mathrm{~b}$. Lower than expected current smoking prevalence was generally observed, especially in studies with hospitalised samples. Former smoking prevalence was typically higher than expected prevalence when reported. National smoking prevalence estimates used for comparison are presented in Supplementary table 3. 


\section{Prevalence of current smoking in included studies}

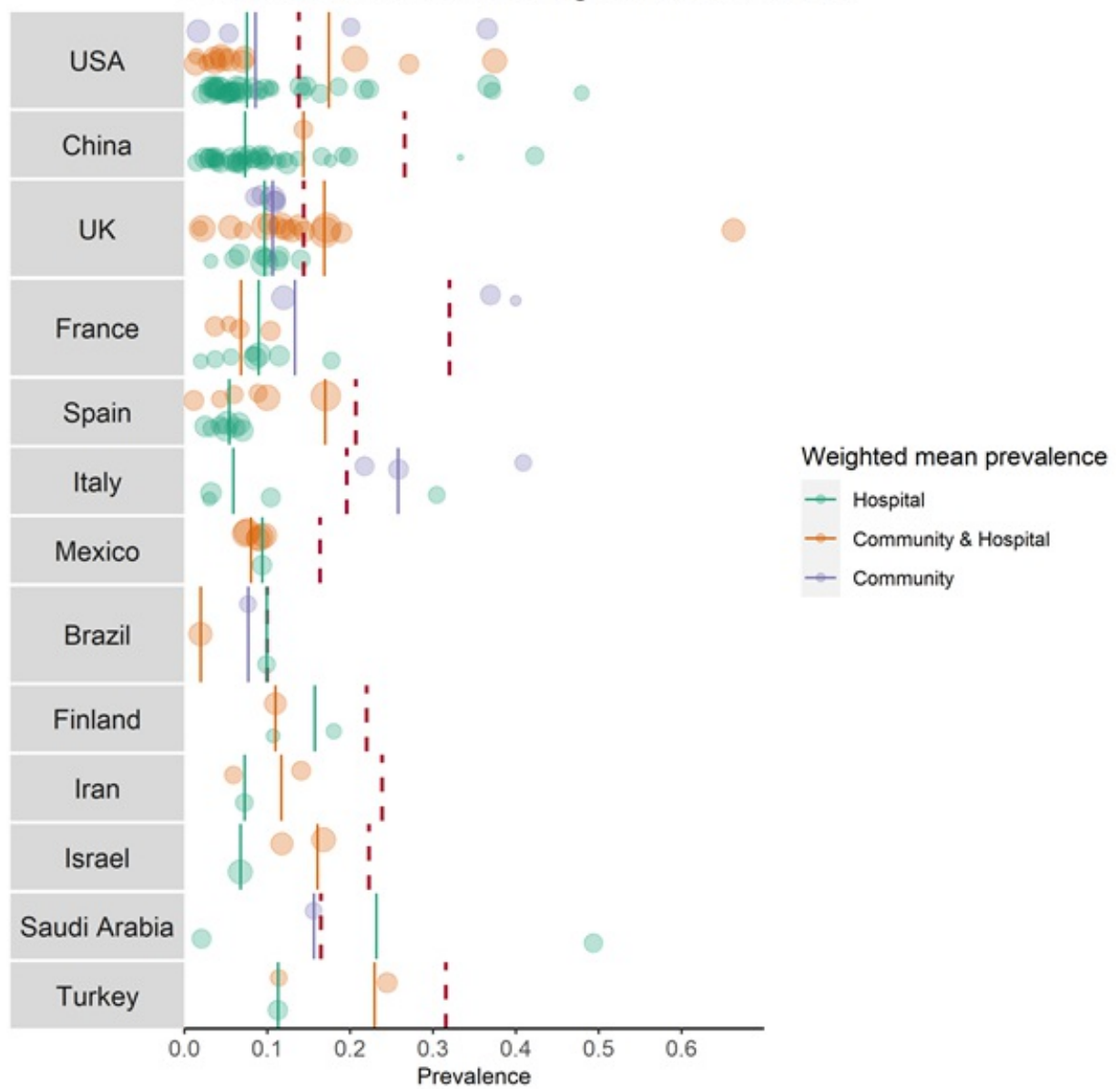

Figure 2a. Weighted mean prevalence of current smoking in included studies, split by country (solid lines). The circles represent individual studies, with colour corresponding to study setting (i.e. community, community and hospital, hospital) and size corresponding to relative study sample size. For comparison, national current smoking prevalence is shown by the dashed red lines. Countries with three or more eligible studies are shown. 


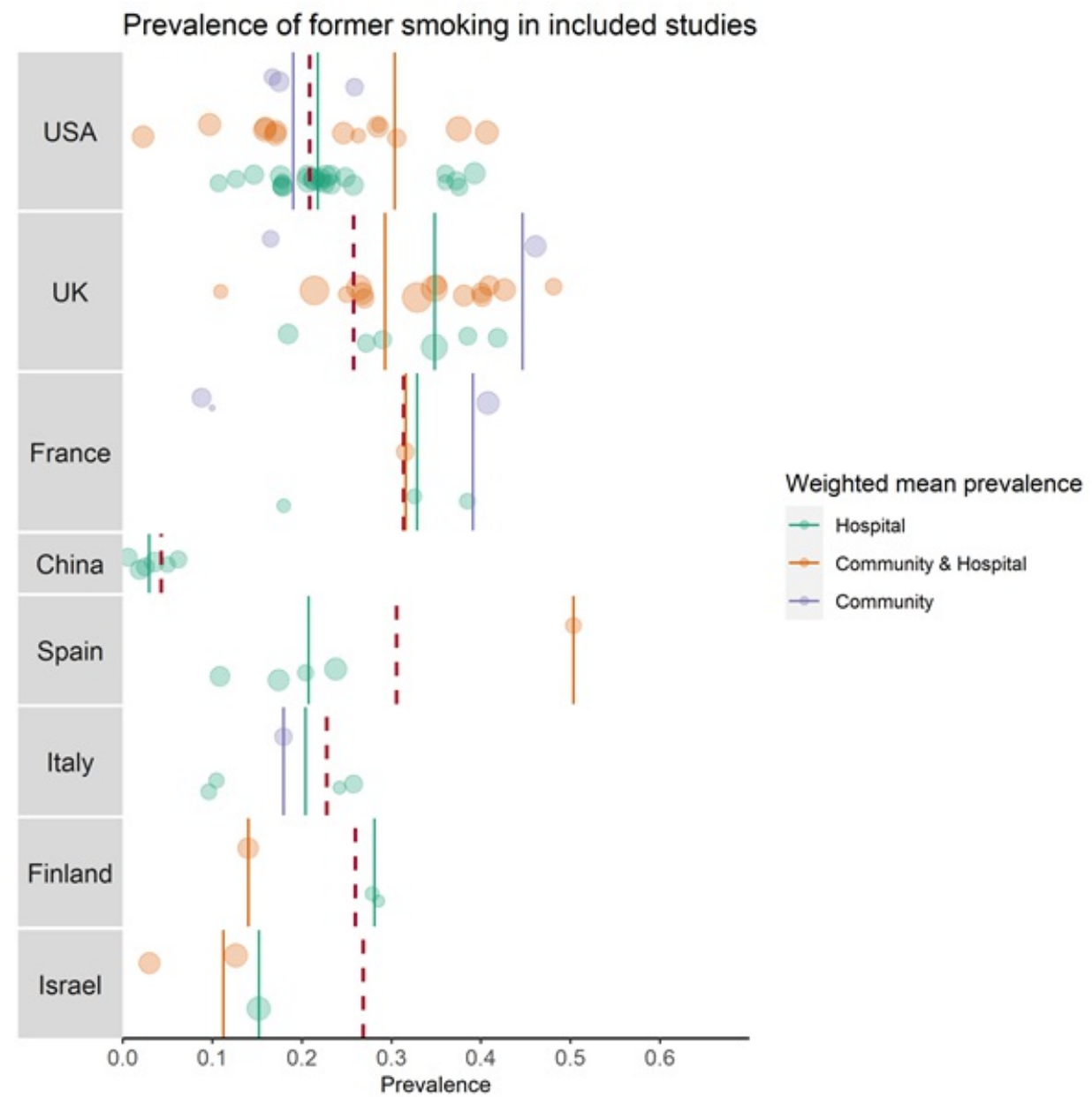

Figure 2b. Prevalence of former smoking in included studies, split by country (solid lines). The circles represent individual studies, with colour corresponding to study setting (i.e. community, community and hospital, hospital) and size corresponding to relative study sample size. For comparison, national former smoking prevalence is shown by dashed red lines. Countries with three or more eligible studies are shown.

\section{SARS-CoV-2 testing by smoking status}

Three studies provided data on access to SARS-CoV-2 diagnostic testing for those meeting local testing criteria by smoking status. In a cohort study of US military veterans aged $54-75^{32}$, current smokers were more likely to receive a test: $42.3 \%(1,603 / 3,789)$ of the sample were current smokers compared with $23.8 \%$ of all veterans aged 50 + years using any tobacco product between 2010-2015 33 . In the UK Biobank cohort ${ }^{34}$, a multivariable analysis showed former $(\mathrm{RR}=1.29$, $95 \% \mathrm{Cl}=1.14-1.45, p<.001)$ and current $(\mathrm{RR}=1.44,95 \% \mathrm{Cl}=1.20-1.71, p<.001)$ compared with never smokers to be more likely to receive a test. In an Australian rapid assessment screening clinic for COVID-19 ${ }^{35}, 9.4 \%(397 / 4,226)$ of the self-referred sample (subsequently assessed by a healthcare professional to decide on testing) were current smokers. Of these self-referrals, healthcare professionals decided that current compared with former or never smokers were less likely to require a test $(\mathrm{RR}=0.93,95 \% \mathrm{Cl}=0.86-1.0, p=0.045)$. 


\section{SARS-CoV-2 infection by smoking status}

Sixty-three studies provided data on SARS-CoV-2 infection for people meeting local testing criteria by smoking status (see Table 2). Meta-analyses were performed for two 'good' and 23 'fair' quality studies (see Figure 3 and 4). Current smokers were at reduced risk of testing positive for SARS-CoV-2 compared with never smokers ( $R R=0.69,95 \%$ Credible Interval $(\mathrm{Crl})=0.58-0.82, \tau=0.36)$. The probability of current smokers being at reduced risk of infection compared with never smokers (RR $\leq 0.9)$ was $99.8 \%$. Former compared with never smokers were at increased risk of testing positive, but data were inconclusive $(\mathrm{RR}=1.03,95 \% \mathrm{Crl}=0.94-1.13, \tau=0.18)$ and favoured there being no important association. The probability of former smokers being at increased risk of infection ( $R R \geq 1.1)$ compared with never smokers was $7.8 \%$. Results were materially unchanged in the two sensitivity analyses.

Table 2. SARS-CoV-2 infection by smoking status.

\begin{tabular}{|c|c|c|c|c|c|c|c|c|c|c|c|}
\hline \multirow[b]{2}{*}{ Author } & \multirow[b]{2}{*}{$\begin{array}{l}\text { Total } \\
\text { population } \\
\text { tested }\end{array}$} & \multicolumn{6}{|c|}{ SARS-CoV-2 negative } & \multicolumn{4}{|c|}{ SARS-CoV-2 positive } \\
\hline & & $\mathrm{N}(\%)$ & $\begin{array}{l}\text { Current } \\
\text { smoker } \\
(\%)\end{array}$ & $\begin{array}{l}\text { Former } \\
\text { smoker } \\
(\%)\end{array}$ & $\begin{array}{l}\text { Current/former } \\
\text { smoker (\%) }\end{array}$ & $\begin{array}{l}\text { Never } \\
\text { smoker } \\
(\%)\end{array}$ & $\begin{array}{l}\text { Not } \\
\text { stated } \\
(\%)\end{array}$ & $\mathrm{N}(\%)$ & $\begin{array}{l}\text { Current } \\
\text { smoker } \\
(\%)\end{array}$ & $\begin{array}{l}\text { Former } \\
\text { smoker } \\
(\%)\end{array}$ & $\begin{array}{l}\text { Current/former } \\
\text { smoker (\%) }\end{array}$ \\
\hline Rentsch & 3528 & $\begin{array}{l}2974 \\
(84.30 \%)\end{array}$ & $\begin{array}{l}1444 \\
(48.55 \%)\end{array}$ & $\begin{array}{l}704 \\
(23.67 \%)\end{array}$ & - & $\begin{array}{l}826 \\
(27.77 \%)\end{array}$ & - & $\begin{array}{l}554 \\
(15.70 \%)\end{array}$ & $\begin{array}{l}159 \\
(28.70 \%)\end{array}$ & $\begin{array}{l}179 \\
(32.31 \%)\end{array}$ & - \\
\hline Fontanet & 661 & $\begin{array}{l}490 \\
(74.13 \%)\end{array}$ & $\begin{array}{l}64 \\
(13.06 \%)\end{array}$ & - & - & $\begin{array}{l}426 \\
(86.94 \%)\end{array}$ & - & $\begin{array}{l}171 \\
(25.87 \%)\end{array}$ & $\begin{array}{l}5 \\
(2.92 \%)\end{array}$ & - & - \\
\hline Cho & 1331 & $\begin{array}{l}793 \\
(59.58 \%)\end{array}$ & $\begin{array}{l}142 \\
(17.91 \%)\end{array}$ & $\begin{array}{l}214 \\
(26.99 \%)\end{array}$ & - & $\begin{array}{l}437 \\
(55.11 \%)\end{array}$ & - & $\begin{array}{l}538 \\
(40.42 \%)\end{array}$ & $\begin{array}{l}111 \\
(20.63 \%)\end{array}$ & $\begin{array}{l}145 \\
(26.95 \%)\end{array}$ & - \\
\hline Shah & 243 & $\begin{array}{l}212 \\
(87.24 \%)\end{array}$ & $\begin{array}{l}52 \\
(24.53 \%)\end{array}$ & $\begin{array}{l}47 \\
(22.17 \%)\end{array}$ & - & $\begin{array}{l}113 \\
(53.30 \%)\end{array}$ & - & $\begin{array}{l}29 \\
(11.93 \%)\end{array}$ & $\begin{array}{l}0 \\
(0.00 \%)\end{array}$ & $\begin{array}{l}9 \\
(31.03 \%)\end{array}$ & - \\
\hline Kolin & 1474 & $\begin{array}{l}805 \\
(54.61 \%)\end{array}$ & $\begin{array}{l}141 \\
(17.52 \%)\end{array}$ & $\begin{array}{l}307 \\
(38.14 \%)\end{array}$ & - & $\begin{array}{l}354 \\
(43.98 \%)\end{array}$ & $3(0.37 \%)$ & $\begin{array}{l}669 \\
(45.39 \%)\end{array}$ & $\begin{array}{l}72 \\
(10.76 \%)\end{array}$ & $\begin{array}{l}285 \\
(42.60 \%)\end{array}$ & - \\
\hline de Lusignan & 3291 & $\begin{array}{l}2740 \\
(83.26 \%)\end{array}$ & $\begin{array}{l}366 \\
(13.36 \%)\end{array}$ & $\begin{array}{l}1450 \\
(52.92 \%)\end{array}$ & - & $\begin{array}{l}924 \\
(33.72 \%)\end{array}$ & - & $\begin{array}{l}551 \\
(16.74 \%)\end{array}$ & $\begin{array}{l}47 \\
(8.53 \%)\end{array}$ & $\begin{array}{l}303 \\
(54.99 \%)\end{array}$ & - \\
\hline Valenti & 789 & $\begin{array}{l}689 \\
(87.33 \%)\end{array}$ & $\begin{array}{l}197 \\
(28.59 \%)\end{array}$ & - & - & - & $\begin{array}{l}492 \\
(71.41 \%)\end{array}$ & $\begin{array}{l}40 \\
(5.07 \%)\end{array}$ & $\begin{array}{l}7 \\
(17.50 \%)\end{array}$ & - & - \\
\hline Parrotta & 76 & $\begin{array}{l}39 \\
(51.32 \%)\end{array}$ & $\begin{array}{l}1 \\
(2.56 \%)\end{array}$ & $\begin{array}{l}10 \\
(25.64 \%)\end{array}$ & - & $\begin{array}{l}27 \\
(69.23 \%)\end{array}$ & $1(2.56 \%)$ & $\begin{array}{l}37 \\
(48.68 \%)\end{array}$ & $\begin{array}{l}1 \\
(2.70 \%)\end{array}$ & $\begin{array}{l}10 \\
(27.03 \%)\end{array}$ & - \\
\hline Berumen & 102875 & $\begin{array}{l}71353 \\
(69.36 \%)\end{array}$ & - & - & $7173(10.05 \%)$ & $\begin{array}{l}64180 \\
(89.95 \%)\end{array}$ & - & $\begin{array}{l}31522 \\
(30.64 \%)\end{array}$ & - & - & $2748(8.72 \%)$ \\
\hline Israel & 24906 & $\begin{array}{l}20755 \\
(83.33 \%)\end{array}$ & $\begin{array}{l}3783 \\
(18.23 \%)\end{array}$ & $\begin{array}{l}2671 \\
(12.87 \%)\end{array}$ & - & $\begin{array}{l}14301 \\
(68.90 \%)\end{array}$ & - & $\begin{array}{l}41151 \\
(165.23 \%)\end{array}$ & $\begin{array}{l}406 \\
(0.99 \%)\end{array}$ & $\begin{array}{l}483 \\
(1.17 \%)\end{array}$ & - \\
\hline del Valle & 1108 & $\begin{array}{l}143 \\
(12.91 \%)\end{array}$ & $\begin{array}{l}27 \\
(18.88 \%)\end{array}$ & $\begin{array}{l}53 \\
(37.06 \%)\end{array}$ & - & - & $\begin{array}{l}63 \\
(44.06 \%)\end{array}$ & $\begin{array}{l}965 \\
(87.09 \%)\end{array}$ & $\begin{array}{l}55 \\
(5.70 \%)\end{array}$ & $\begin{array}{l}293 \\
(30.36 \%)\end{array}$ & - \\
\hline Romao & 34 & $\begin{array}{l}20 \\
(58.82 \%)\end{array}$ & - & - & $5(25.00 \%)$ & - & $\begin{array}{l}15 \\
(75.00 \%)\end{array}$ & $\begin{array}{l}14 \\
(41.18 \%)\end{array}$ & - & - & $4(28.57 \%)$ \\
\hline Ramlall & 11116 & $\begin{array}{l}4723 \\
(42.49 \%)\end{array}$ & - & - & - & - & - & $\begin{array}{l}6393 \\
(57.51 \%)\end{array}$ & - & - & $\begin{array}{l}1643.001 \\
(25.70 \%)\end{array}$ \\
\hline Sharma & 501 & $\begin{array}{l}267 \\
(53.29 \%)\end{array}$ & - & - & $1(0.37 \%)$ & - & $\begin{array}{l}266 \\
(99.63 \%)\end{array}$ & $\begin{array}{l}234 \\
(46.71 \%)\end{array}$ & - & - & $20(8.55 \%)$ \\
\hline Eugen-Olsen & 407 & $\begin{array}{l}290 \\
(71.25 \%)\end{array}$ & $\begin{array}{l}76 \\
(26.21 \%)\end{array}$ & $\begin{array}{l}104 \\
(35.86 \%)\end{array}$ & - & $\begin{array}{l}102 \\
(35.17 \%)\end{array}$ & - & $\begin{array}{l}117 \\
(28.75 \%)\end{array}$ & $\begin{array}{l}8 \\
(6.84 \%)\end{array}$ & $\begin{array}{l}46 \\
(39.32 \%)\end{array}$ & - \\
\hline
\end{tabular}




\begin{tabular}{|c|c|c|c|c|c|c|c|c|c|c|c|}
\hline & & & & & & & & & & & \\
\hline $\begin{array}{l}\text { Raisi- } \\
\text { Estabragh }\end{array}$ & 4510 & $\begin{array}{l}3184 \\
(70.60 \%)\end{array}$ & - & - & 1653 (51.92\%) & - & $\begin{array}{l}1531 \\
(48.08 \%)\end{array}$ & $\begin{array}{l}1326 \\
(29.40 \%)\end{array}$ & - & - & $683(51.51 \%)$ \\
\hline Houlihan & 177 & $\begin{array}{l}97 \\
(54.80 \%)\end{array}$ & $\begin{array}{l}14 \\
(14.43 \%)\end{array}$ & $\begin{array}{l}14 \\
(14.43 \%)\end{array}$ & - & $\begin{array}{l}69 \\
(71.13 \%)\end{array}$ & - & $\begin{array}{l}80 \\
(45.20 \%)\end{array}$ & $\begin{array}{l}7 \\
(8.75 \%)\end{array}$ & $\begin{array}{l}19 \\
(23.75 \%)\end{array}$ & - \\
\hline McQueenie & 428199 & $\begin{array}{l}424355 \\
(99.10 \%)\end{array}$ & - & - & $\begin{array}{l}189299 \\
(44.61 \%)\end{array}$ & $\begin{array}{l}235056 \\
(55.39 \%)\end{array}$ & - & $\begin{array}{l}1311 \\
(0.31 \%)\end{array}$ & - & - & 669 (51.03\%) \\
\hline Woolford & 4474 & $\begin{array}{l}3161 \\
(70.65 \%)\end{array}$ & $\begin{array}{l}441 \\
(13.95 \%)\end{array}$ & $\begin{array}{l}1194 \\
(37.77 \%)\end{array}$ & - & $\begin{array}{l}1526 \\
(48.28 \%)\end{array}$ & - & $\begin{array}{l}1313 \\
(29.35 \%)\end{array}$ & $\begin{array}{l}145 \\
(11.04 \%)\end{array}$ & $\begin{array}{l}525 \\
(39.98 \%)\end{array}$ & - \\
\hline Lan & 104 & $\begin{array}{l}83 \\
(79.81 \%)\end{array}$ & - & - & $24(28.92 \%)$ & - & $\begin{array}{l}59 \\
(71.08 \%)\end{array}$ & $\begin{array}{l}21 \\
(20.19 \%)\end{array}$ & - & - & $1(4.76 \%)$ \\
\hline $\begin{array}{l}\text { Hernandez- } \\
\text { Garduno }\end{array}$ & 32583 & $\begin{array}{l}20279 \\
(62.24 \%)\end{array}$ & - & - & 2399 (11.83\%) & $\begin{array}{l}17861 \\
(88.08 \%)\end{array}$ & - & $\begin{array}{l}12304 \\
(37.76 \%)\end{array}$ & - & - & 1191 (9.68\%) \\
\hline Govind & 6215 & $\begin{array}{l}6207 \\
(99.87 \%)\end{array}$ & $\begin{array}{l}4104 \\
(66.12 \%)\end{array}$ & $\begin{array}{l}1669 \\
(26.89 \%)\end{array}$ & - & $\begin{array}{l}342 \\
(5.51 \%)\end{array}$ & - & $\begin{array}{l}102 \\
(1.64 \%)\end{array}$ & $\begin{array}{l}78 \\
(76.47 \%)\end{array}$ & $\begin{array}{l}20 \\
(19.61 \%)\end{array}$ & - \\
\hline $\mathrm{Gu}$ & 4699 & $\begin{array}{l}3815 \\
(81.19 \%)\end{array}$ & $\begin{array}{l}360 \\
(9.44 \%)\end{array}$ & $\begin{array}{l}1142 \\
(29.93 \%)\end{array}$ & - & $\begin{array}{l}2313 \\
(60.63 \%)\end{array}$ & - & $\begin{array}{l}884 \\
(18.81 \%)\end{array}$ & $\begin{array}{l}40 \\
(4.52 \%)\end{array}$ & $\begin{array}{l}264 \\
(29.86 \%)\end{array}$ & - \\
\hline Kibler & 702 & $\begin{array}{l}680 \\
(96.87 \%)\end{array}$ & $\begin{array}{l}25 \\
(3.68 \%)\end{array}$ & - & - & - & $\begin{array}{l}655 \\
(96.32 \%)\end{array}$ & $\begin{array}{l}22 \\
(3.13 \%)\end{array}$ & $\begin{array}{l}1 \\
(4.55 \%)\end{array}$ & - & - \\
\hline Petrilli & 10620 & $\begin{array}{l}5341 \\
(50.29 \%)\end{array}$ & $\begin{array}{l}3454 \\
(64.67 \%)\end{array}$ & $\begin{array}{l}816 \\
(15.28 \%)\end{array}$ & - & $\begin{array}{l}541 \\
(10.13 \%)\end{array}$ & $\begin{array}{l}530 \\
(9.92 \%)\end{array}$ & $\begin{array}{l}5279 \\
(49.71 \%)\end{array}$ & $\begin{array}{l}3268 \\
(61.91 \%)\end{array}$ & $\begin{array}{l}902 \\
(17.09 \%)\end{array}$ & - \\
\hline $\begin{array}{l}\text { Bello- } \\
\text { Chavolla }\end{array}$ & 150200 & $\begin{array}{l}98567 \\
(65.62 \%)\end{array}$ & - & - & 9624 (9.76\%) & - & $\begin{array}{l}88943 \\
(90.24 \%)\end{array}$ & $\begin{array}{l}51633 \\
(34.38 \%)\end{array}$ & - & - & $4366(8.46 \%)$ \\
\hline Auvinen & 61 & $\begin{array}{l}33 \\
(54.10 \%)\end{array}$ & $\begin{array}{l}10 \\
(30.30 \%)\end{array}$ & $\begin{array}{l}8 \\
(24.24 \%)\end{array}$ & - & $\begin{array}{l}15 \\
(45.45 \%)\end{array}$ & - & $\begin{array}{l}28 \\
(45.90 \%)\end{array}$ & $\begin{array}{l}1 \\
(3.57 \%)\end{array}$ & $\begin{array}{l}9 \\
(32.14 \%)\end{array}$ & - \\
\hline Favara & 70 & $\begin{array}{l}55 \\
(78.57 \%)\end{array}$ & $\begin{array}{l}5 \\
(9.09 \%)\end{array}$ & - & - & - & $\begin{array}{l}50 \\
(90.91 \%)\end{array}$ & $\begin{array}{l}15 \\
(21.43 \%)\end{array}$ & $\begin{array}{l}2 \\
(13.33 \%)\end{array}$ & - & - \\
\hline Antonio-Villa & 34263 & $\begin{array}{l}23338 \\
(68.11 \%)\end{array}$ & $\begin{array}{l}2293 \\
(9.83 \%)\end{array}$ & - & - & - & $\begin{array}{l}21045 \\
(90.17 \%)\end{array}$ & $\begin{array}{l}10925 \\
(31.89 \%)\end{array}$ & $\begin{array}{l}1023 \\
(9.36 \%)\end{array}$ & - & - \\
\hline Merzon & 7807 & $\begin{array}{l}7025 \\
(89.98 \%)\end{array}$ & - & - & $1136(16.17 \%)$ & - & $\begin{array}{l}5889 \\
(83.83 \%)\end{array}$ & $\begin{array}{l}782 \\
(10.02 \%)\end{array}$ & - & - & 127 (16.24\%) \\
\hline Trubiano & 2676 & $\begin{array}{l}2827 \\
(105.64 \%)\end{array}$ & - & - & 256 (9.06\%) & - & $\begin{array}{l}2586 \\
(91.48 \%)\end{array}$ & $\begin{array}{l}108 \\
(4.04 \%)\end{array}$ & - & - & 3 (2.78\%) \\
\hline $\begin{array}{l}\text { Shi, } \\
\text { Resurreccion }\end{array}$ & 1521 & $\begin{array}{l}1265 \\
(83.17 \%)\end{array}$ & - & - & $681(53.83 \%)$ & - & $\begin{array}{l}584 \\
(46.17 \%)\end{array}$ & $\begin{array}{l}256 \\
(16.83 \%)\end{array}$ & - & - & 154 (60.16\%) \\
\hline Riley & 120620 & $\begin{array}{l}120461 \\
(99.87 \%)\end{array}$ & $\begin{array}{l}2594 \\
(2.15 \%)\end{array}$ & - & - & $\begin{array}{l}19914 \\
(16.53 \%)\end{array}$ & $\begin{array}{l}97953 \\
(81.32 \%)\end{array}$ & $\begin{array}{l}159 \\
(0.13 \%)\end{array}$ & $\begin{array}{l}3 \\
(1.89 \%)\end{array}$ & - & - \\
\hline Alizadehsani & 319 & $\begin{array}{l}196 \\
(61.44 \%)\end{array}$ & - & - & - & - & $\begin{array}{l}196 \\
(100.00 \%)\end{array}$ & $\begin{array}{l}123 \\
(38.56 \%)\end{array}$ & - & - & $1(0.81 \%)$ \\
\hline Merkely & 10474 & $\begin{array}{l}10336 \\
(98.68 \%)\end{array}$ & $\begin{array}{l}2904 \\
(28.10 \%)\end{array}$ & $\begin{array}{l}2107 \\
(20.39 \%)\end{array}$ & - & $\begin{array}{l}5310 \\
(51.37 \%)\end{array}$ & $\begin{array}{l}15 \\
(0.15 \%)\end{array}$ & $\begin{array}{l}70 \\
(0.67 \%)\end{array}$ & $\begin{array}{l}16 \\
(22.86 \%)\end{array}$ & $\begin{array}{l}15 \\
(21.43 \%)\end{array}$ & - \\
\hline Mcgrail & 209 & $\begin{array}{l}118 \\
(56.46 \%)\end{array}$ & - & - & 31 (26.27\%) & - & $\begin{array}{l}87 \\
(73.73 \%)\end{array}$ & $\begin{array}{l}91 \\
(43.54 \%)\end{array}$ & - & - & $8(8.79 \%)$ \\
\hline Izquierdo & 71192 & $\begin{array}{l}\text { NA ( } \\
\text { NA\%) }\end{array}$ & - & - & - & - & - & $\begin{array}{l}1006 \\
(1.41 \%)\end{array}$ & $\begin{array}{l}111 \\
(11.03 \%)\end{array}$ & - & - \\
\hline Ward & 99908 & $\begin{array}{l}94416 \\
(94.50 \%)\end{array}$ & $\begin{array}{l}10202 \\
(10.81 \%)\end{array}$ & - & - & - & $\begin{array}{l}84214 \\
(89.19 \%)\end{array}$ & $\begin{array}{l}5492 \\
(5.50 \%)\end{array}$ & $\begin{array}{l}433 \\
(7.88 \%)\end{array}$ & - & - \\
\hline Ebinger & 6062 & $\begin{array}{l}5850 \\
(96.50 \%)\end{array}$ & $\begin{array}{l}99 \\
(1.69 \%)\end{array}$ & - & - & - & $\begin{array}{l}5668 \\
(96.89 \%)\end{array}$ & $\begin{array}{l}212 \\
(3.50 \%)\end{array}$ & $\begin{array}{l}3 \\
(1.42 \%)\end{array}$ & - & - \\
\hline Salerno & 15920 & $\begin{array}{l}14753 \\
(92.67 \%)\end{array}$ & - & - & 5517 (37.40\%) & $\begin{array}{l}8278 \\
(56.11 \%)\end{array}$ & $\begin{array}{l}958 \\
(6.49 \%)\end{array}$ & $\begin{array}{l}1167 \\
(7.33 \%)\end{array}$ & - & - & 339 \\
\hline
\end{tabular}




\begin{tabular}{|c|c|c|c|c|c|c|c|c|c|c|c|}
\hline Iversen & 28792 & $\begin{array}{l}27629 \\
(95.96 \%)\end{array}$ & $\begin{array}{l}4430 \\
(16.03 \%)\end{array}$ & $\begin{array}{l}1799 \\
(6.51 \%)\end{array}$ & - & $\begin{array}{l}21217 \\
(76.79 \%)\end{array}$ & $\begin{array}{l}246 \\
(0.89 \%)\end{array}$ & $\begin{array}{l}1163 \\
(4.04 \%)\end{array}$ & $\begin{array}{l}177 \\
(15.22 \%)\end{array}$ & $\begin{array}{l}78 \\
(6.71 \%)\end{array}$ & - \\
\hline $\begin{array}{l}\text { Hippisley- } \\
\text { Cox }\end{array}$ & 8275949 & $\begin{array}{l}\text { NA( } \\
\text { NA\%) }\end{array}$ & - & - & - & - & - & $\begin{array}{l}19486 \\
(0.24 \%)\end{array}$ & $\begin{array}{l}1354 \\
(6.95 \%)\end{array}$ & $\begin{array}{l}5715 \\
(29.33 \%)\end{array}$ & - \\
\hline Fillmore & 22914 & $\begin{array}{l}21120 \\
(92.17 \%)\end{array}$ & $\begin{array}{l}8137 \\
(38.53 \%)\end{array}$ & $\begin{array}{l}8416 \\
(39.85 \%)\end{array}$ & - & $\begin{array}{l}3227 \\
(15.28 \%)\end{array}$ & $\begin{array}{l}1340 \\
(6.34 \%)\end{array}$ & $\begin{array}{l}1794 \\
(7.83 \%)\end{array}$ & $\begin{array}{l}452 \\
(25.20 \%)\end{array}$ & $\begin{array}{l}899 \\
(50.11 \%)\end{array}$ & - \\
\hline Alkurt & 119 & $\begin{array}{l}\text { NA( } \\
\text { NA\%) }\end{array}$ & - & - & - & - & - & $\begin{array}{l}119 \\
(100.00 \%)\end{array}$ & $\begin{array}{l}14 \\
(11.76 \%)\end{array}$ & - & - \\
\hline Ariza & 351 & $\begin{array}{l}322 \\
(91.74 \%)\end{array}$ & $\begin{array}{l}21 \\
(6.52 \%)\end{array}$ & - & - & - & $\begin{array}{l}301 \\
(93.48 \%)\end{array}$ & $\begin{array}{l}29 \\
(8.26 \%)\end{array}$ & $\begin{array}{l}3 \\
(10.34 \%)\end{array}$ & - & - \\
\hline Carrat & 14393 & $\begin{array}{l}13426 \\
(93.28 \%)\end{array}$ & $\begin{array}{l}1652 \\
(12.30 \%)\end{array}$ & $\begin{array}{l}5620 \\
(41.86 \%)\end{array}$ & - & $\begin{array}{l}6154 \\
(45.84 \%)\end{array}$ & - & $\begin{array}{l}967 \\
(6.72 \%)\end{array}$ & $\begin{array}{l}98 \\
(10.13 \%)\end{array}$ & $\begin{array}{l}353 \\
(36.50 \%)\end{array}$ & - \\
\hline Meini & 461 & $\begin{array}{l}243 \\
(52.71 \%)\end{array}$ & $\begin{array}{l}39 \\
(16.05 \%)\end{array}$ & $\begin{array}{l}66 \\
(27.16 \%)\end{array}$ & - & $\begin{array}{l}138 \\
(56.79 \%)\end{array}$ & - & $\begin{array}{l}218 \\
(47.29 \%)\end{array}$ & $\begin{array}{l}9 \\
(4.13 \%)\end{array}$ & $\begin{array}{l}53 \\
(24.31 \%)\end{array}$ & - \\
\hline Favara & 434 & $\begin{array}{l}354 \\
(81.57 \%)\end{array}$ & $\begin{array}{l}28 \\
(7.91 \%)\end{array}$ & - & - & - & $\begin{array}{l}326 \\
(92.09 \%)\end{array}$ & $\begin{array}{l}80 \\
(18.43 \%)\end{array}$ & $\begin{array}{l}9 \\
(11.25 \%)\end{array}$ & - & - \\
\hline Erber & 4554 & $\begin{array}{l}4446 \\
(97.63 \%)\end{array}$ & - & - & $806(18.13 \%)$ & - & $\begin{array}{l}3640 \\
(81.87 \%)\end{array}$ & $\begin{array}{l}108 \\
(2.37 \%)\end{array}$ & - & - & $11(10.19 \%)$ \\
\hline Roederer & 815 & $\begin{array}{l}390 \\
(47.85 \%)\end{array}$ & $\begin{array}{l}175 \\
(44.87 \%)\end{array}$ & $\begin{array}{l}32 \\
(8.21 \%)\end{array}$ & - & $\begin{array}{l}183 \\
(46.92 \%)\end{array}$ & - & $\begin{array}{l}425 \\
(52.15 \%)\end{array}$ & $\begin{array}{l}127 \\
(29.88 \%)\end{array}$ & $\begin{array}{l}40 \\
(9.41 \%)\end{array}$ & - \\
\hline Makaronidis & 567 & $\begin{array}{l}127 \\
(22.40 \%)\end{array}$ & $\begin{array}{l}16 \\
(12.60 \%)\end{array}$ & - & - & - & $\begin{array}{l}111 \\
(87.40 \%)\end{array}$ & $\begin{array}{l}440 \\
(77.60 \%)\end{array}$ & $\begin{array}{l}37 \\
(8.41 \%)\end{array}$ & - & - \\
\hline loannou & 88747 & $\begin{array}{l}78616 \\
(88.58 \%)\end{array}$ & $\begin{array}{l}17138 \\
(21.80 \%)\end{array}$ & $\begin{array}{l}29245 \\
(37.20 \%)\end{array}$ & - & $\begin{array}{l}22327 \\
(28.40 \%)\end{array}$ & $\begin{array}{l}9906 \\
(12.60 \%)\end{array}$ & $\begin{array}{l}10131 \\
(11.42 \%)\end{array}$ & $\begin{array}{l}1135 \\
(11.20 \%)\end{array}$ & $\begin{array}{l}4073 \\
(40.20 \%)\end{array}$ & - \\
\hline Perico & 423 & $\begin{array}{l}260 \\
(61.47 \%)\end{array}$ & $\begin{array}{l}69 \\
(26.54 \%)\end{array}$ & $\begin{array}{l}35 \\
(13.46 \%)\end{array}$ & - & $\begin{array}{l}156 \\
(60.00 \%)\end{array}$ & - & $\begin{array}{l}163 \\
(38.53 \%)\end{array}$ & $\begin{array}{l}23 \\
(14.11 \%)\end{array}$ & $\begin{array}{l}41 \\
(25.15 \%)\end{array}$ & - \\
\hline Vila-Corcoles & 2,324 & $\begin{array}{l}1944 \\
(83.65 \%)\end{array}$ & - & - & - & - & - & $\begin{array}{l}380 \\
(16.35 \%)\end{array}$ & - & - & $27(7.11 \%)$ \\
\hline O'Reilly & 1,334 & $\begin{array}{l}1284 \\
(96.25 \%)\end{array}$ & - & - & 376 (29.28\%) & - & $\begin{array}{l}908 \\
(70.72 \%)\end{array}$ & $\begin{array}{l}50 \\
(3.75 \%)\end{array}$ & - & - & $4(8.00 \%)$ \\
\hline Martini & 146 & $\begin{array}{l}108 \\
(73.97 \%)\end{array}$ & - & - & 56 (51.85\%) & $\begin{array}{l}52 \\
(48.15 \%)\end{array}$ & - & $\begin{array}{l}38 \\
(26.03 \%)\end{array}$ & - & - & 12 (31.58\%) \\
\hline Ghinai & 1,435 & $\begin{array}{l}1004 \\
(69.97 \%)\end{array}$ & $\begin{array}{l}412 \\
(41.04 \%)\end{array}$ & $\begin{array}{l}155 \\
(15.44 \%)\end{array}$ & - & $\begin{array}{l}341 \\
(33.96 \%)\end{array}$ & $\begin{array}{l}96 \\
(9.56 \%)\end{array}$ & $\begin{array}{l}431 \\
(30.03 \%)\end{array}$ & $\begin{array}{l}113 \\
(26.22 \%)\end{array}$ & $\begin{array}{l}96 \\
(22.27 \%)\end{array}$ & - \\
\hline Kortela & 2,993 & $\begin{array}{l}2419 \\
(80.82 \%)\end{array}$ & $\begin{array}{l}300 \\
(12.40 \%)\end{array}$ & $\begin{array}{l}340 \\
(14.06 \%)\end{array}$ & - & $\begin{array}{l}636 \\
(26.29 \%)\end{array}$ & $\begin{array}{l}1143 \\
(47.25 \%)\end{array}$ & $\begin{array}{l}574 \\
(19.18 \%)\end{array}$ & $\begin{array}{l}26 \\
(4.53 \%)\end{array}$ & $\begin{array}{l}80 \\
(13.94 \%)\end{array}$ & - \\
\hline Gallichotte & 239 & $\begin{array}{l}190 \\
(79.50 \%)\end{array}$ & $\begin{array}{l}40 \\
(21.05 \%)\end{array}$ & $\begin{array}{l}28 \\
(14.74 \%)\end{array}$ & - & $\begin{array}{l}110 \\
(57.89 \%)\end{array}$ & - & $\begin{array}{l}49 \\
(20.50 \%)\end{array}$ & $\begin{array}{l}8 \\
(16.33 \%)\end{array}$ & $\begin{array}{l}12 \\
(24.49 \%)\end{array}$ & - \\
\hline Saeed & 173 & $\begin{array}{l}69 \\
(39.88 \%)\end{array}$ & $\begin{array}{l}2 \\
(2.90 \%)\end{array}$ & - & - & - & $\begin{array}{l}67 \\
(97.10 \%)\end{array}$ & $\begin{array}{l}104 \\
(60.12 \%)\end{array}$ & $\begin{array}{l}9 \\
(8.65 \%)\end{array}$ & - & - \\
\hline Woolcott & $1,636,050$ & $\begin{array}{l}878840 \\
(53.72 \%)\end{array}$ & - & - & 85816 (9.76\%) & - & $\begin{array}{l}793024 \\
(90.24 \%)\end{array}$ & $\begin{array}{l}757210 \\
(46.28 \%)\end{array}$ & - & - & 57451 (7.59\%) \\
\hline Barasa & 394 & $\begin{array}{l}277 \\
(70.30 \%)\end{array}$ & $\begin{array}{l}49 \\
(17.69 \%)\end{array}$ & $\begin{array}{l}105 \\
(37.91 \%)\end{array}$ & - & $\begin{array}{l}110 \\
(39.71 \%)\end{array}$ & $\begin{array}{l}13 \\
(4.69 \%)\end{array}$ & $\begin{array}{l}117 \\
(29.70 \%)\end{array}$ & $\begin{array}{l}9 \\
(7.69 \%)\end{array}$ & $\begin{array}{l}37 \\
(31.62 \%)\end{array}$ & - \\
\hline
\end{tabular}

Note. Niedzwiedz et al. reported on SARS-CoV-2 infection by smoking status in multivariable analyses but did not present raw data. 


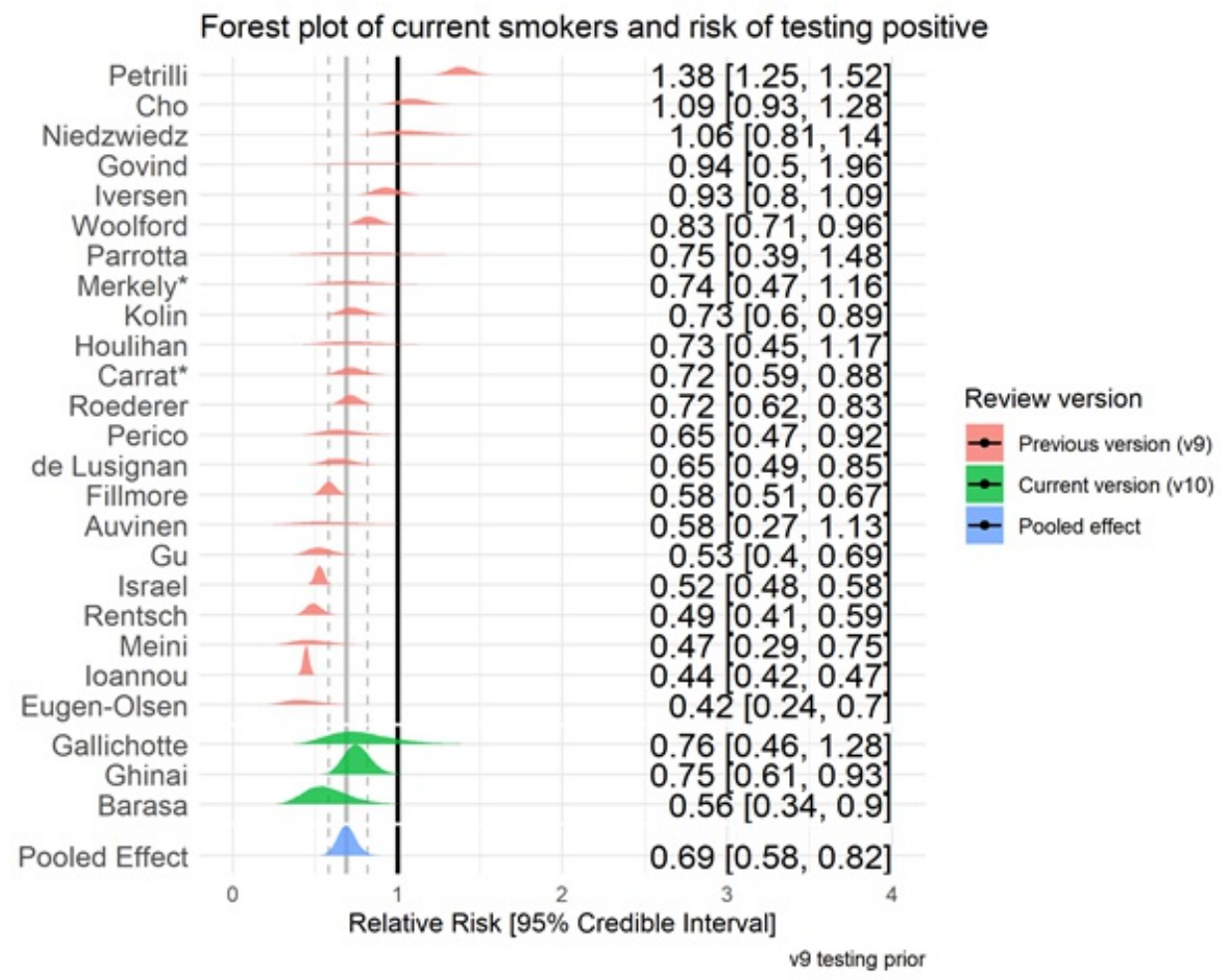

Figure 3. Forest plot for risk of testing positive for SARS-CoV-2 in current vs. never smokers. * Indicates 'good' quality studies. The prior from the previous review version (v9) was $R R=0.69$.

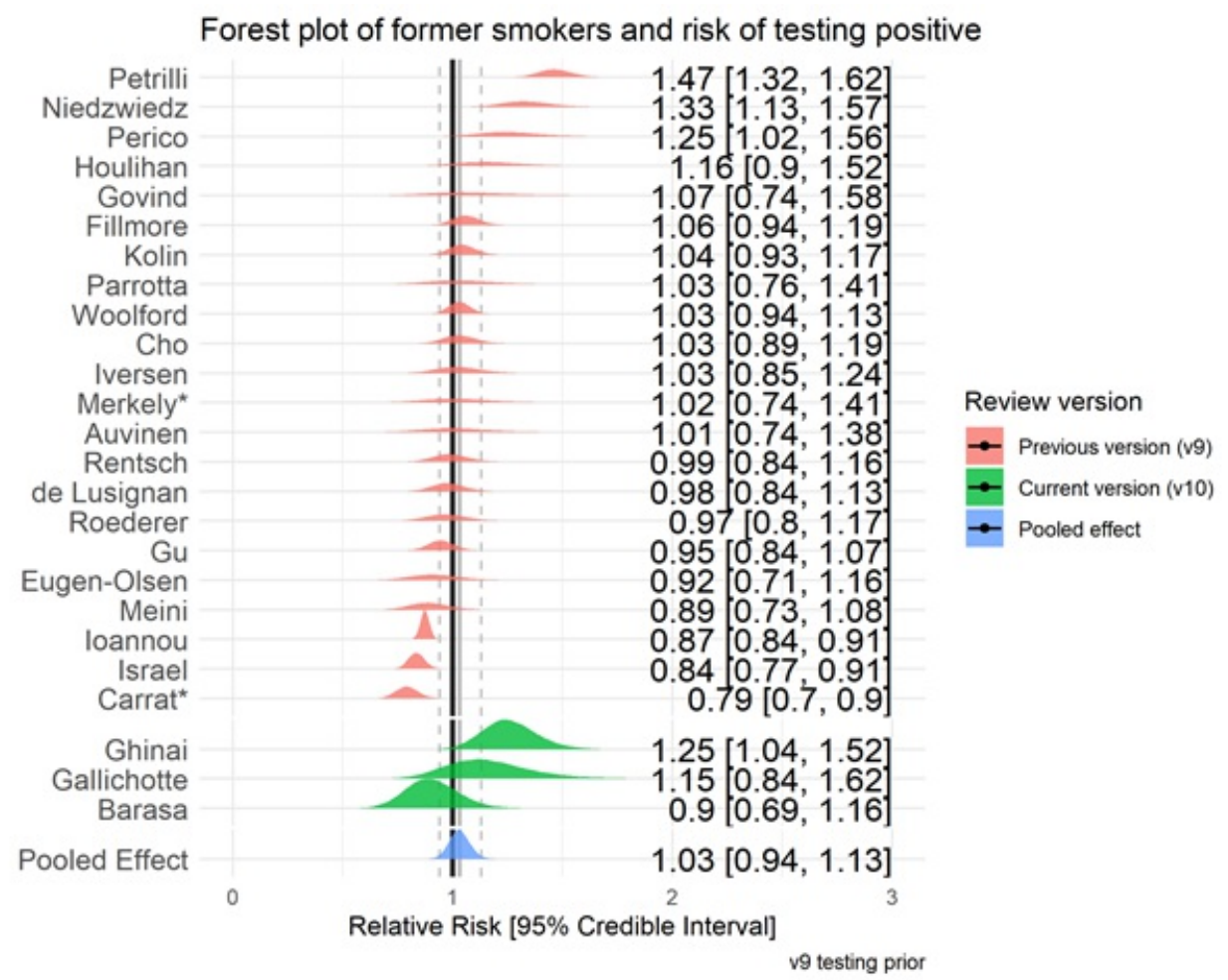

Figure 4. Forest plot for risk of testing positive for SARS-CoV-2 in former vs. never smokers. * Indicates 'good' quality studies. The prior from the previous review version (v9) was $R R=1.02$. 
Forty-one studies examined hospitalisation for COVID-19 disease, stratified by smoking status (see Table 3). Metaanalyses were performed for 13 'fair' quality studies (see Figure 5 and 6$)$. Current $(R R=1.08, C r l=0.95-1.23, \tau=0.18$ ) and former $(\mathrm{RR}=1.18, \mathrm{Crl}=1.07-1.31, \tau=0.14)$ compared with never smokers were at increased risk of hospitalisation with COVID-19. However, data for current smokers were inconclusive and favoured there being no important association. The probability of current and former smokers being at increased risk of hospitalisation ( $R R \geq 1.1)$ compared with never smokers was $31 \%$ and $89 \%$, respectively. Results were materially unchanged in two sensitivity analyses.

Table 3. Hospitalisation with COVID-19 by smoking status.

\begin{tabular}{|c|c|c|c|c|c|c|c|c|c|c|c|c|}
\hline \multirow[b]{2}{*}{ Author } & \multirow[b]{2}{*}{$\begin{array}{l}\text { Population } \\
\text { with } \\
\text { outcome }\end{array}$} & \multicolumn{7}{|c|}{ Community } & \multicolumn{4}{|c|}{ Hospitalised } \\
\hline & & N (\%) & $\begin{array}{l}\text { Current } \\
\text { smoker } \\
(\%)\end{array}$ & $\begin{array}{l}\text { Former } \\
\text { smoker } \\
(\%)\end{array}$ & $\begin{array}{l}\text { Current/former } \\
\text { smoker (\%) }\end{array}$ & $\begin{array}{l}\text { Never } \\
\text { smoker } \\
(\%)\end{array}$ & $\begin{array}{l}\text { Never/unknown } \\
\text { smoker (\%) }\end{array}$ & $\begin{array}{l}\text { Not } \\
\text { stated } \\
(\%)\end{array}$ & N (\%) & $\begin{array}{l}\text { Current } \\
\text { smoker } \\
(\%)\end{array}$ & $\begin{array}{l}\text { Former } \\
\text { smoker } \\
(\%)\end{array}$ & $\begin{array}{l}\text { Cur } \\
\text { sme }\end{array}$ \\
\hline Rentsch & 554 & $\begin{array}{l}269 \\
(48 \%)\end{array}$ & $\begin{array}{l}69 \\
(25.65 \%)\end{array}$ & $\begin{array}{l}90 \\
(33.46 \%)\end{array}$ & - & $\begin{array}{l}110 \\
(40.89 \%)\end{array}$ & - & - & $\begin{array}{l}285 \\
(51 \%)\end{array}$ & $\begin{array}{l}90 \\
(31.58 \%)\end{array}$ & $\begin{array}{l}89 \\
(31.23 \%)\end{array}$ & - \\
\hline $\begin{array}{l}\text { Chow (US } \\
\text { CDC) }\end{array}$ & 6637 & $\begin{array}{l}5143 \\
(77 \%)\end{array}$ & $\begin{array}{l}61 \\
(1.19 \%)\end{array}$ & $\begin{array}{l}80 \\
(1.56 \%)\end{array}$ & - & - & - & $\begin{array}{l}5002 \\
(97.26 \%)\end{array}$ & $\begin{array}{l}1494 \\
(22 \%)\end{array}$ & $\begin{array}{l}27 \\
(1.81 \%)\end{array}$ & $\begin{array}{l}78 \\
(5.22 \%)\end{array}$ & - \\
\hline Argenziano & 1000 & $\begin{array}{l}151 \\
(15 \%)\end{array}$ & $\begin{array}{l}14 \\
(9.27 \%)\end{array}$ & $\begin{array}{l}18 \\
(11.92 \%)\end{array}$ & - & $\begin{array}{l}119 \\
(78.81 \%)\end{array}$ & - & - & $\begin{array}{l}849 \\
(84 \%)\end{array}$ & $\begin{array}{l}35 \\
(4.12 \%)\end{array}$ & $\begin{array}{l}161 \\
(18.96 \%)\end{array}$ & - \\
\hline Lubetzky & 54 & $\begin{array}{l}15 \\
(27 \%)\end{array}$ & - & - & $4(26.67 \%)$ & - & - & $\begin{array}{l}11 \\
(73.33 \%)\end{array}$ & $\begin{array}{l}39 \\
(72 \%)\end{array}$ & - & - & $8(2$ \\
\hline Carillo-Vega & 9946 & $\begin{array}{l}3922 \\
(39 \%)\end{array}$ & $\begin{array}{l}408 \\
(10.40 \%)\end{array}$ & - & - & - & - & $\begin{array}{l}3514 \\
(89.60 \%)\end{array}$ & $\begin{array}{l}6024 \\
(60 \%)\end{array}$ & $\begin{array}{l}486 \\
(8.07 \%)\end{array}$ & - & - \\
\hline Yanover & 4353 & $\begin{array}{l}4180 \\
(96 \%)\end{array}$ & $\begin{array}{l}484 \\
(11.58 \%)\end{array}$ & $\begin{array}{l}118 \\
(2.82 \%)\end{array}$ & - & $\begin{array}{l}3578 \\
(85.60 \%)\end{array}$ & - & - & $\begin{array}{l}173 \\
(3 \%)\end{array}$ & $\begin{array}{l}30 \\
(17.34 \%)\end{array}$ & $\begin{array}{l}11 \\
(6.36 \%)\end{array}$ & - \\
\hline Hamer & 387109 & $\begin{array}{l}386349 \\
(99 \%)\end{array}$ & $\begin{array}{l}37333 \\
(9.66 \%)\end{array}$ & $\begin{array}{l}134542 \\
(34.82 \%)\end{array}$ & - & $\begin{array}{l}214474 \\
(55.51 \%)\end{array}$ & - & - & $\begin{array}{l}760 \\
(0 \%)\end{array}$ & $\begin{array}{l}93 \\
(12.24 \%)\end{array}$ & $\begin{array}{l}313 \\
(41.18 \%)\end{array}$ & - \\
\hline Heili-Frades & 4712 & $\begin{array}{l}1973 \\
(41 \%)\end{array}$ & $\begin{array}{l}121 \\
(6.13 \%)\end{array}$ & $\begin{array}{l}222 \\
(11.25 \%)\end{array}$ & - & - & $1630(82.62 \%)$ & $\begin{array}{l}1630 \\
(82.62 \%)\end{array}$ & $\begin{array}{l}2739 \\
(58 \%)\end{array}$ & $\begin{array}{l}112 \\
(4.09 \%)\end{array}$ & $\begin{array}{l}598 \\
(21.83 \%)\end{array}$ & - \\
\hline Freites & 123 & $\begin{array}{l}69 \\
(56 \%)\end{array}$ & $\begin{array}{l}1 \\
(1.45 \%)\end{array}$ & - & - & - & - & $\begin{array}{l}68 \\
(98.55 \%)\end{array}$ & $\begin{array}{l}54 \\
(43 \%)\end{array}$ & $\begin{array}{l}3 \\
(5.56 \%)\end{array}$ & - & - \\
\hline Berumen & 102875 & $\begin{array}{c}18832 \\
(18 \%)\end{array}$ & - & - & $1546(8.21 \%)$ & - & $17286(91.79 \%)$ & - & $\begin{array}{l}12690 \\
(12 \%)\end{array}$ & - & - & 120 \\
\hline Gianfrancesco & 600 & $\begin{array}{l}323 \\
(53 \%)\end{array}$ & - & - & $61(18.89 \%)$ & - & - & $\begin{array}{l}262 \\
(81.11 \%)\end{array}$ & $\begin{array}{l}277 \\
(46 \%)\end{array}$ & - & - & 68( \\
\hline Chaudhry & 40 & $\begin{array}{l}19 \\
(47 \%)\end{array}$ & - & - & $0(0.00 \%)$ & - & - & $\begin{array}{l}19 \\
(100.00 \%)\end{array}$ & $\begin{array}{l}21 \\
(52 \%)\end{array}$ & - & - & $6(2$ \\
\hline Giannouchos & 89756 & $\begin{array}{l}58485 \\
(65 \%)\end{array}$ & $\begin{array}{l}4679 \\
(8.00 \%)\end{array}$ & - & - & - & $53806(92.00 \%)$ & - & $\begin{array}{l}31271 \\
(34 \%)\end{array}$ & $\begin{array}{l}2721 \\
(8.70 \%)\end{array}$ & - & - \\
\hline $\begin{array}{l}\text { Wang, } \\
\text { Oekelen }\end{array}$ & 57 & $\begin{array}{l}22 \\
(38 \%)\end{array}$ & - & - & $6(27.27 \%)$ & - & - & $\begin{array}{l}16 \\
(72.73 \%)\end{array}$ & $\begin{array}{l}36 \\
(63 \%)\end{array}$ & - & - & 15( \\
\hline Miyara & 470 & $\begin{array}{l}132 \\
(28 \%)\end{array}$ & $\begin{array}{l}14 \\
(10.61 \%)\end{array}$ & $\begin{array}{l}41 \\
(31.06 \%)\end{array}$ & - & $\begin{array}{l}77 \\
(58.33 \%)\end{array}$ & - & - & $\begin{array}{l}338 \\
(71 \%)\end{array}$ & $\begin{array}{l}18 \\
(5.33 \%)\end{array}$ & $\begin{array}{l}111 \\
(32.84 \%)\end{array}$ & - \\
\hline Suleyman & 463 & $\begin{array}{l}108 \\
(23 \%)\end{array}$ & - & - & $23(21.30 \%)$ & - & - & $\begin{array}{l}85 \\
(78.70 \%)\end{array}$ & $\begin{array}{l}355 \\
(76 \%)\end{array}$ & - & - & 137 \\
\hline Garassino & 196 & $\begin{array}{l}48 \\
(24 \%)\end{array}$ & $\begin{array}{l}10 \\
(20.83 \%)\end{array}$ & $\begin{array}{l}27 \\
(56.25 \%)\end{array}$ & - & $\begin{array}{l}11 \\
(22.92 \%)\end{array}$ & - & - & $\begin{array}{l}152 \\
(77 \%)\end{array}$ & $\begin{array}{l}38 \\
(25.00 \%)\end{array}$ & $\begin{array}{l}84 \\
(55.26 \%)\end{array}$ & - \\
\hline
\end{tabular}




\begin{tabular}{|c|c|c|c|c|c|c|c|c|c|c|c|c|}
\hline Siso-Almirall & 260 & $\begin{array}{l}119 \\
(45 \%)\end{array}$ & - & - & $31(26.05 \%)$ & - & - & $\begin{array}{l}88 \\
(73.95 \%)\end{array}$ & $\begin{array}{l}141 \\
(54 \%)\end{array}$ & - & - & 50( \\
\hline $\mathrm{Gu}$ & 884 & $\begin{array}{l}511 \\
(57 \%)\end{array}$ & $\begin{array}{l}30 \\
(5.87 \%)\end{array}$ & $\begin{array}{l}126 \\
(24.66 \%)\end{array}$ & - & $\begin{array}{l}355 \\
(69.47 \%)\end{array}$ & - & - & $\begin{array}{l}373 \\
(42 \%)\end{array}$ & $\begin{array}{l}10 \\
(2.68 \%)\end{array}$ & $\begin{array}{l}138 \\
(37.00 \%)\end{array}$ & - \\
\hline Killerby & 531 & $\begin{array}{l}311 \\
(58 \%)\end{array}$ & - & - & 37 (11.90\%) & $\begin{array}{l}222 \\
(71.38 \%)\end{array}$ & - & $\begin{array}{l}52 \\
(16.72 \%)\end{array}$ & $\begin{array}{l}220 \\
(41 \%)\end{array}$ & - & - & 54( \\
\hline Petrilli & 5279 & $\begin{array}{l}2538 \\
(48 \%)\end{array}$ & $\begin{array}{l}147 \\
(5.79 \%)\end{array}$ & $\begin{array}{l}337 \\
(13.28 \%)\end{array}$ & - & $\begin{array}{l}1678 \\
(66.12 \%)\end{array}$ & - & $\begin{array}{l}376 \\
(14.81 \%)\end{array}$ & $\begin{array}{l}2741 \\
(51 \%)\end{array}$ & $\begin{array}{l}141 \\
(5.14 \%)\end{array}$ & $\begin{array}{l}565 \\
(20.61 \%)\end{array}$ & - \\
\hline Nguyen & 689 & $\begin{array}{l}333 \\
(48 \%)\end{array}$ & - & - & $57(17.12 \%)$ & - & - & $\begin{array}{l}276 \\
(82.88 \%)\end{array}$ & $\begin{array}{l}356 \\
(51 \%)\end{array}$ & - & - & 114 \\
\hline Mendy & 689 & $\begin{array}{l}473 \\
(68 \%)\end{array}$ & - & - & $84(17.76 \%)$ & - & - & $\begin{array}{l}389 \\
(82.24 \%)\end{array}$ & $\begin{array}{l}216 \\
(31 \%)\end{array}$ & - & - & 86( \\
\hline Soares & 10713 & $\begin{array}{l}9561 \\
(89 \%)\end{array}$ & $\begin{array}{l}132 \\
(1.38 \%)\end{array}$ & - & - & - & $9429(98.62 \%)$ & - & $\begin{array}{l}1152 \\
(10 \%)\end{array}$ & $\begin{array}{l}77 \\
(6.68 \%)\end{array}$ & - & - \\
\hline Zobairy & 203 & $\begin{array}{l}65 \\
(32 \%)\end{array}$ & $\begin{array}{l}1 \\
(1.54 \%)\end{array}$ & - & - & - & 64 (98.46\%) & - & $\begin{array}{l}138 \\
(67 \%)\end{array}$ & $\begin{array}{l}11 \\
(7.97 \%)\end{array}$ & - & - \\
\hline Izquierdo & 1006 & $\begin{array}{l}743 \\
(73 \%)\end{array}$ & $\begin{array}{l}52 \\
(7.00 \%)\end{array}$ & - & - & - & 691 (93.00\%) & - & $\begin{array}{l}263 \\
(26 \%)\end{array}$ & $\begin{array}{l}16 \\
(6.08 \%)\end{array}$ & - & - \\
\hline Rizzo & 76819 & $\begin{array}{l}60039 \\
(78 \%)\end{array}$ & $\begin{array}{l}3931 \\
(6.55 \%)\end{array}$ & $\begin{array}{l}11379 \\
(18.95 \%)\end{array}$ & - & $\begin{array}{l}30042 \\
(50.04 \%)\end{array}$ & - & $\begin{array}{l}14687 \\
(24.46 \%)\end{array}$ & $\begin{array}{l}16780 \\
(21 \%)\end{array}$ & $\begin{array}{l}1254 \\
(7.47 \%)\end{array}$ & $\begin{array}{l}4585 \\
(27.32 \%)\end{array}$ & - \\
\hline Dashti & 4140 & $\begin{array}{l}2759 \\
(66 \%)\end{array}$ & - & - & $600(21.75 \%)$ & $\begin{array}{l}1541 \\
(55.85 \%)\end{array}$ & - & $\begin{array}{l}618 \\
(22.40 \%)\end{array}$ & $\begin{array}{l}1381 \\
(33 \%)\end{array}$ & - & - & 577 \\
\hline Pan & 12084 & $\begin{array}{l}8548 \\
(70 \%)\end{array}$ & - & - & $1263(14.78 \%)$ & - & - & $\begin{array}{l}7285 \\
(85.22 \%)\end{array}$ & $\begin{array}{l}3536 \\
(29 \%)\end{array}$ & - & - & 874 \\
\hline Vilar-Garcia & 328892 & $\begin{array}{l}291254 \\
(88 \%)\end{array}$ & $\begin{array}{l}64792 \\
(22.25 \%)\end{array}$ & - & - & - & - & $\begin{array}{l}226462 \\
(77.75 \%)\end{array}$ & $\begin{array}{l}37638 \\
(11 \%)\end{array}$ & $\begin{array}{l}9526 \\
(25.31 \%)\end{array}$ & - & - \\
\hline lbarra-Nava & 416546 & $\begin{array}{l}302693 \\
(72 \%)\end{array}$ & $\begin{array}{l}26773 \\
(8.84 \%)\end{array}$ & - & - & - & - & $\begin{array}{l}275920 \\
(91.16 \%)\end{array}$ & $\begin{array}{l}113853 \\
(27 \%)\end{array}$ & $\begin{array}{l}8875 \\
(7.80 \%)\end{array}$ & - & - \\
\hline Dashti & 12347 & $\begin{array}{l}8946 \\
(72 \%)\end{array}$ & $\begin{array}{l}353 \\
(3.95 \%)\end{array}$ & $\begin{array}{l}1099 \\
(12.28 \%)\end{array}$ & - & $\begin{array}{l}5133 \\
(57.38 \%)\end{array}$ & - & $\begin{array}{l}2361 \\
(26.39 \%)\end{array}$ & $\begin{array}{l}3401 \\
(27 \%)\end{array}$ & $\begin{array}{l}210 \\
(6.17 \%)\end{array}$ & $\begin{array}{l}860 \\
(25.29 \%)\end{array}$ & - \\
\hline Da Silva Neto & 91 & $\begin{array}{l}44 \\
(48 \%)\end{array}$ & - & - & $4(9.09 \%)$ & - & $40(90.91 \%)$ & - & $\begin{array}{l}47 \\
(51 \%)\end{array}$ & - & - & 14( \\
\hline $\begin{array}{l}\text { Israel, } \\
\text { Schaffer }\end{array}$ & 26676 & $\begin{array}{l}13706 \\
(51 \%)\end{array}$ & $\begin{array}{l}944 \\
(6.89 \%)\end{array}$ & $\begin{array}{l}2166 \\
(15.80 \%)\end{array}$ & - & $\begin{array}{l}10596 \\
(77.31 \%)\end{array}$ & - & - & $\begin{array}{l}12970 \\
(48 \%)\end{array}$ & $\begin{array}{l}880 \\
(6.78 \%)\end{array}$ & $\begin{array}{l}1936 \\
(14.93 \%)\end{array}$ & - \\
\hline Ioannou & 10131 & $\begin{array}{l}6624 \\
(65 \%)\end{array}$ & $\begin{array}{l}716 \\
(10.81 \%)\end{array}$ & $\begin{array}{l}2484 \\
(37.50 \%)\end{array}$ & - & $\begin{array}{l}2542 \\
(38.38 \%)\end{array}$ & - & $\begin{array}{l}882 \\
(13.32 \%)\end{array}$ & $\begin{array}{l}3507 \\
(34 \%)\end{array}$ & $\begin{array}{l}419 \\
(11.95 \%)\end{array}$ & $\begin{array}{l}1593 \\
(45.42 \%)\end{array}$ & - \\
\hline Zhang, Li & 1,596 & $\begin{array}{l}576 \\
(36 \%)\end{array}$ & $\begin{array}{l}63 \\
(10.94 \%)\end{array}$ & $\begin{array}{l}190 \\
(32.99 \%)\end{array}$ & - & $\begin{array}{l}318 \\
(55.21 \%)\end{array}$ & - & $5(0.87 \%)$ & $\begin{array}{l}1020 \\
(63 \%)\end{array}$ & $\begin{array}{l}116 \\
(11.37 \%)\end{array}$ & $\begin{array}{l}429 \\
(42.06 \%)\end{array}$ & - \\
\hline $\begin{array}{l}\text { Parra- } \\
\text { Bracamonte }\end{array}$ & 331,298 & $\begin{array}{l}235840 \\
(71 \%)\end{array}$ & - & - & 16676 (7.07\%) & - & - & $\begin{array}{l}219164 \\
(92.93 \%)\end{array}$ & $\begin{array}{l}95458 \\
(28 \%)\end{array}$ & - & - & 751 \\
\hline Jehi & 4,536 & $\begin{array}{l}3578 \\
(78 \%)\end{array}$ & $\begin{array}{l}247 \\
(6.90 \%)\end{array}$ & $\begin{array}{l}943 \\
(26.36 \%)\end{array}$ & - & $\begin{array}{l}1795 \\
(50.17 \%)\end{array}$ & - & $\begin{array}{l}593 \\
(16.57 \%)\end{array}$ & $\begin{array}{l}958 \\
(21 \%)\end{array}$ & $\begin{array}{l}82 \\
(8.56 \%)\end{array}$ & $\begin{array}{l}349 \\
(36.43 \%)\end{array}$ & - \\
\hline Ilic & 107 & $\begin{array}{l}69 \\
(64 \%)\end{array}$ & $\begin{array}{l}21 \\
(30.43 \%)\end{array}$ & - & - & - & - & $\begin{array}{l}48 \\
(69.57 \%)\end{array}$ & $\begin{array}{l}38 \\
(35 \%)\end{array}$ & $\begin{array}{l}11 \\
(28.95 \%)\end{array}$ & - & - \\
\hline Arleo & 70 & $\begin{array}{l}36 \\
(51 \%)\end{array}$ & $\begin{array}{l}1 \\
(2.78 \%)\end{array}$ & $\begin{array}{l}10 \\
(27.78 \%)\end{array}$ & - & $\begin{array}{l}25 \\
(69.44 \%)\end{array}$ & - & - & $\begin{array}{l}34 \\
(48 \%)\end{array}$ & $\begin{array}{l}0 \\
(0.00 \%)\end{array}$ & $\begin{array}{l}10 \\
(29.41 \%)\end{array}$ & - \\
\hline Kortela & 604 & $\begin{array}{l}246 \\
(40 \%)\end{array}$ & $\begin{array}{l}12 \\
(4.88 \%)\end{array}$ & $\begin{array}{l}14 \\
(5.69 \%)\end{array}$ & - & $\begin{array}{l}55 \\
(22.36 \%)\end{array}$ & - & $\begin{array}{l}165 \\
(67.07 \%)\end{array}$ & $\begin{array}{l}328 \\
(54 \%)\end{array}$ & $\begin{array}{l}14 \\
(4.27 \%)\end{array}$ & $\begin{array}{l}66 \\
(20.12 \%)\end{array}$ & - \\
\hline
\end{tabular}




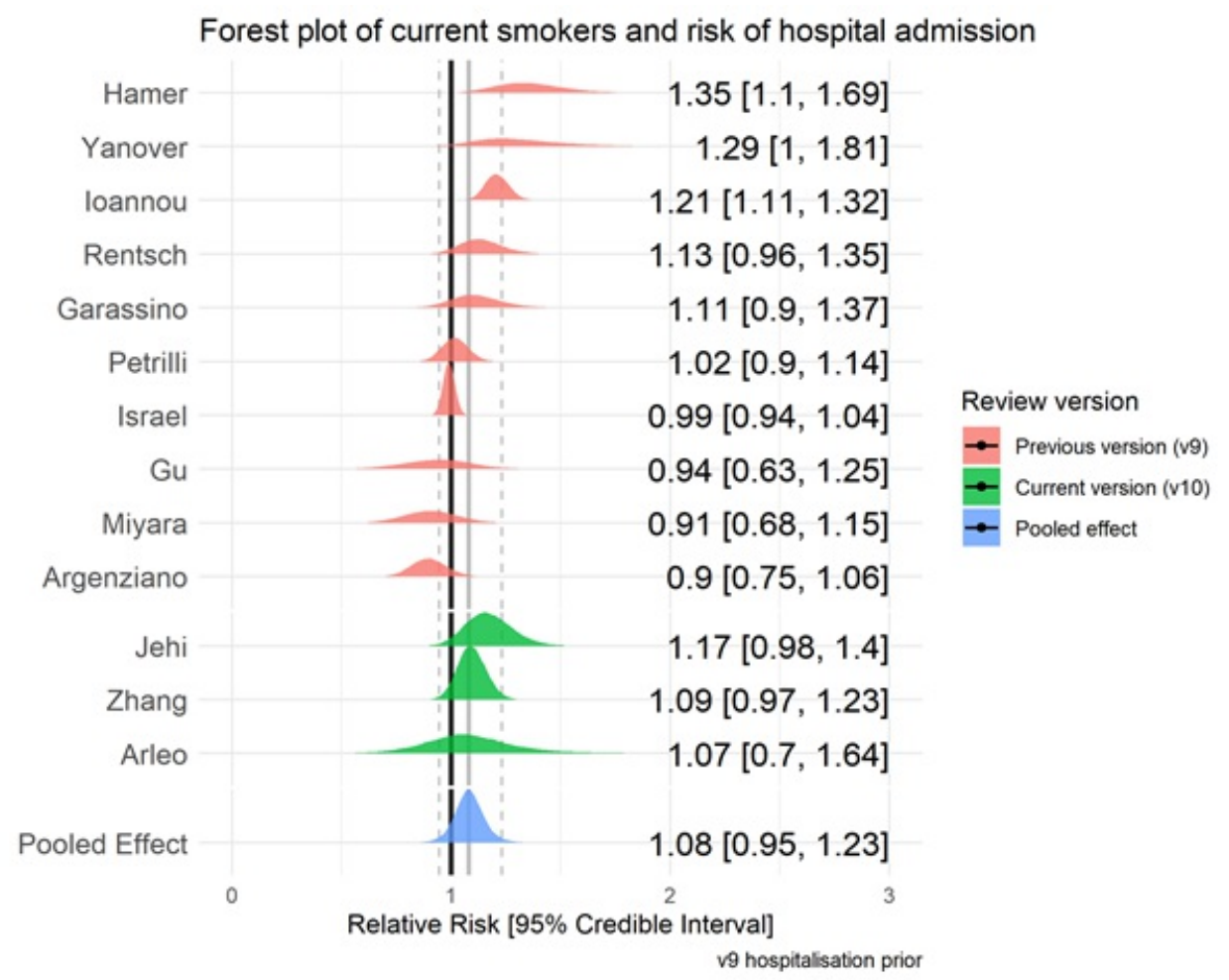

Figure 5. Forest plot for risk of hospitalisation in current vs. never smokers. The prior from the previous review version (v9) was $\mathrm{RR}=1.06$.

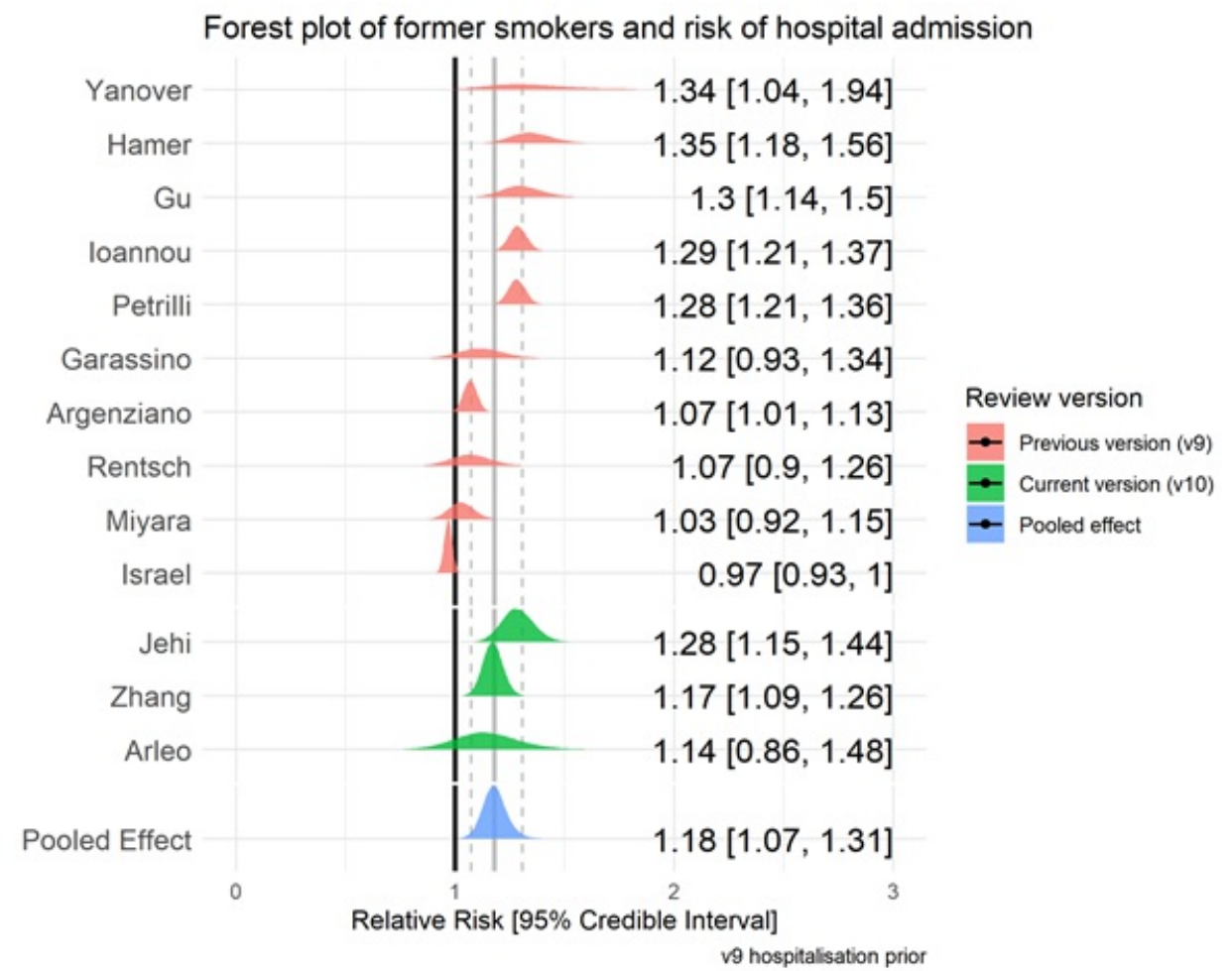

Figure 6. Forest plot for risk of hospitalisation in former vs. never smokers. The prior from the previous version ( $\mathrm{v} 9$ ) was $\mathrm{RR}=1.17$. 


\section{Disease severity by smoking status}

Sixty-five studies reported disease severity in hospitalised patients stratified by smoking status (see Table 4). Severe (as opposed to non-severe) disease was broadly defined as requiring intensive treatment unit (ITU) admission, requiring oxygen as a hospital inpatient or in-hospital death. Meta-analyses were performed for eight 'fair' quality studies (see Figure 7 and 8). Current $(R R=1.26, \mathrm{Crl}=0.86-1.94, \tau=0.34,95 \% \mathrm{Cl}=0.01-0.86)$ and former $(\mathrm{RR}=1.52, \mathrm{Crl}=1.12-2.06, \tau=$ $0.29,95 \% \mathrm{Cl}=0.05-0.65)$ compared with never smokers were at increased risk of greater disease severity; data for current smokers were inconclusive but favoured there being a small but important association. The probability of current and former smokers having increased risk of greater disease severity $(R R \geq 1.1)$ compared with never smokers was $80 \%$ and $98 \%$, respectively. Results were materially unchanged in two sensitivity analyses.

Table 4. Disease severity by smoking status.

\begin{tabular}{|c|c|c|c|c|c|c|c|c|c|c|c|c|}
\hline \multirow[b]{2}{*}{ Author } & \multirow[b]{2}{*}{$\begin{array}{l}\text { Population } \\
\text { with } \\
\text { severity }\end{array}$} & \multicolumn{7}{|c|}{ Non severe disease } & \multicolumn{4}{|c|}{ Severe disease } \\
\hline & & N (\%) & $\begin{array}{l}\text { Current } \\
\text { smoker } \\
(\%)\end{array}$ & $\begin{array}{l}\text { Former } \\
\text { smoker } \\
(\%)\end{array}$ & $\begin{array}{l}\text { Current/former } \\
\text { smoker (\%) }\end{array}$ & $\begin{array}{l}\text { Never } \\
\text { smoker } \\
(\%)\end{array}$ & $\begin{array}{l}\text { Never/unknown } \\
\text { smoker (\%) }\end{array}$ & $\begin{array}{l}\text { Not } \\
\text { stated } \\
(\%)\end{array}$ & $\mathrm{N}(\%)$ & $\begin{array}{l}\text { Current } \\
\text { smoker } \\
(\%)\end{array}$ & $\begin{array}{l}\text { Former } \\
\text { smoker } \\
(\%)\end{array}$ & $\begin{array}{l}\text { C } \\
\text { si }\end{array}$ \\
\hline Guan, $\mathrm{Ni}$ & 1085 & $\begin{array}{l}913 \\
(84 \%)\end{array}$ & $\begin{array}{l}108 \\
(11.83 \%)\end{array}$ & $\begin{array}{l}12 \\
(1.31 \%)\end{array}$ & - & $\begin{array}{l}793 \\
(86.86 \%)\end{array}$ & - & - & $\begin{array}{l}172 \\
(15 \%)\end{array}$ & $\begin{array}{l}29 \\
(16.86 \%)\end{array}$ & $\begin{array}{l}9 \\
(5.23 \%)\end{array}$ & - \\
\hline Zhang, Dong & 9 & $\begin{array}{l}3 \\
(33 \%)\end{array}$ & $0(0.00 \%)$ & $\begin{array}{l}3 \\
(100.00 \%)\end{array}$ & - & $\begin{array}{l}0 \\
(0.00 \%)\end{array}$ & - & - & $\begin{array}{l}6 \\
(66 \%)\end{array}$ & $\begin{array}{l}2 \\
(33.33 \%)\end{array}$ & $\begin{array}{l}4 \\
(66.67 \%)\end{array}$ & - \\
\hline Wan & 9 & $\begin{array}{l}8 \\
(88 \%)\end{array}$ & $\begin{array}{l}8 \\
(100.00 \%)\end{array}$ & $0(0.00 \%)$ & - & $\begin{array}{l}0 \\
(0.00 \%)\end{array}$ & - & - & $\begin{array}{l}1 \\
(11 \%)\end{array}$ & $\begin{array}{l}1 \\
(100.00 \%)\end{array}$ & $\begin{array}{l}0 \\
(0.00 \%)\end{array}$ & - \\
\hline $\begin{array}{l}\text { Huang, } \\
\text { Wang }\end{array}$ & 3 & $\begin{array}{l}3 \\
(100 \%)\end{array}$ & $\begin{array}{l}3 \\
(100.00 \%)\end{array}$ & $0(0.00 \%)$ & - & $\begin{array}{l}0 \\
(0.00 \%)\end{array}$ & - & - & $0(0 \%)$ & $0(-\%)$ & $0(-\%)$ & - \\
\hline Rentsch & 285 & $\begin{array}{l}168 \\
(58 \%)\end{array}$ & $\begin{array}{l}47 \\
(27.98 \%)\end{array}$ & $\begin{array}{l}53 \\
(31.55 \%)\end{array}$ & - & $\begin{array}{l}68 \\
(40.48 \%)\end{array}$ & - & - & $\begin{array}{l}117 \\
(41 \%)\end{array}$ & $\begin{array}{l}43 \\
(36.75 \%)\end{array}$ & $\begin{array}{l}36 \\
(30.77 \%)\end{array}$ & \\
\hline $\mathrm{Hu}$ & 323 & $\begin{array}{l}151 \\
(46 \%)\end{array}$ & - & - & 12 (7.95\%) & - & 139 (92.05\%) & - & $\begin{array}{l}172 \\
(53 \%)\end{array}$ & - & - & $2 t$ \\
\hline Wang, Pan & 125 & $\begin{array}{l}100 \\
(80 \%)\end{array}$ & - & - & $9(9.00 \%)$ & - & $91(91.00 \%)$ & - & $\begin{array}{l}25 \\
(20 \%)\end{array}$ & - & - & 7 \\
\hline Kim & 27 & $\begin{array}{l}21 \\
(77 \%)\end{array}$ & $\begin{array}{l}3 \\
(14.29 \%)\end{array}$ & - & - & - & $18(85.71 \%)$ & - & $\begin{array}{l}6 \\
(22 \%)\end{array}$ & $\begin{array}{l}2 \\
(33.33 \%)\end{array}$ & $\begin{array}{l}0 \\
(0.00 \%)\end{array}$ & - \\
\hline Shi, Yu & 474 & $\begin{array}{l}425 \\
(89 \%)\end{array}$ & - & - & $34(8.00 \%)$ & - & 391 (92.00\%) & - & $\begin{array}{l}49 \\
(10 \%)\end{array}$ & - & - & 6 \\
\hline Liao, Feng & 148 & $\begin{array}{l}92 \\
(62 \%)\end{array}$ & - & - & $5(5.43 \%)$ & - & - & $\begin{array}{l}87 \\
(94.57 \%)\end{array}$ & $\begin{array}{l}56 \\
(37 \%)\end{array}$ & $3(5.36 \%)$ & - & - \\
\hline Shi, Ren & 134 & $\begin{array}{l}88 \\
(65 \%)\end{array}$ & - & - & $8(9.09 \%)$ & - & - & $\begin{array}{l}80 \\
(90.91 \%)\end{array}$ & $\begin{array}{l}46 \\
(34 \%)\end{array}$ & - & - & 6 \\
\hline Hadjadj & 50 & $\begin{array}{l}15 \\
(30 \%)\end{array}$ & $1(6.67 \%)$ & $\begin{array}{l}2 \\
(13.33 \%)\end{array}$ & - & $\begin{array}{l}12 \\
(80.00 \%)\end{array}$ & - & - & $\begin{array}{l}35 \\
(70 \%)\end{array}$ & $0(0.00 \%)$ & $\begin{array}{l}7 \\
(20.00 \%)\end{array}$ & - \\
\hline Zheng, Xiong & 73 & $\begin{array}{l}43 \\
(58 \%)\end{array}$ & - & - & $6(13.95 \%)$ & $\begin{array}{l}37 \\
(86.05 \%)\end{array}$ & - & - & $\begin{array}{l}30 \\
(41 \%)\end{array}$ & - & - & 2 \\
\hline de la Rica & 48 & $\begin{array}{l}26 \\
(54 \%)\end{array}$ & - & - & $6(23.08 \%)$ & - & - & $\begin{array}{l}20 \\
(76.92 \%)\end{array}$ & $\begin{array}{l}20 \\
(41 \%)\end{array}$ & - & - & 4 \\
\hline
\end{tabular}




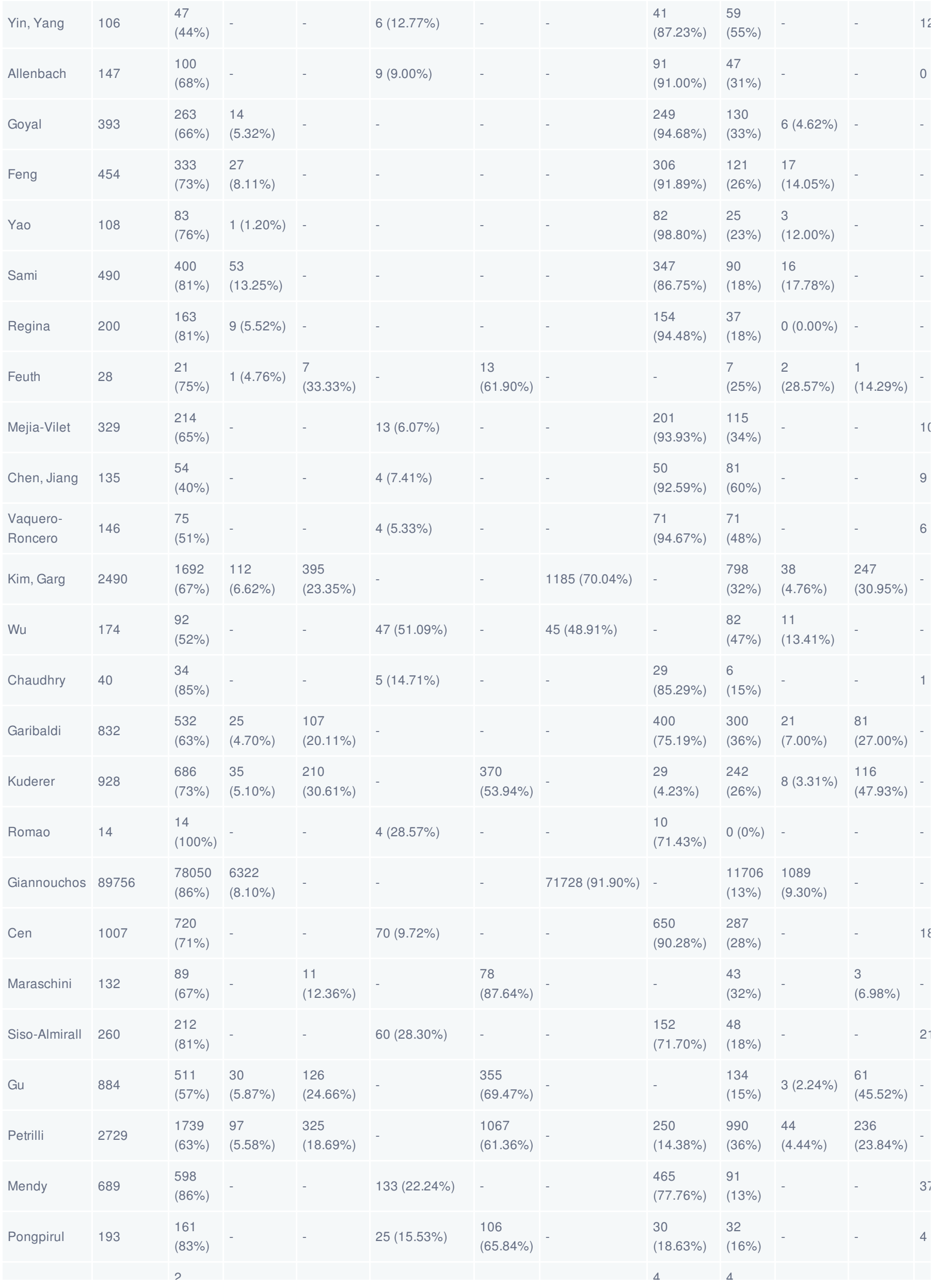




\begin{tabular}{|c|c|c|c|c|c|c|c|c|c|c|c|c|}
\hline Jin, Gu & 6 & (33\%) & - & - & $0(0.00 \%)$ & - & - & (200.00\%) & $(66 \%)$ & - & - & 2 \\
\hline Senkal & 611 & $\begin{array}{l}446 \\
(73 \%)\end{array}$ & $\begin{array}{l}48 \\
(10.76 \%)\end{array}$ & - & - & - & - & $\begin{array}{l}398 \\
(89.24 \%)\end{array}$ & $\begin{array}{l}165 \\
(27 \%)\end{array}$ & $\begin{array}{l}21 \\
(12.73 \%)\end{array}$ & - & - \\
\hline Patel & 129 & $\begin{array}{l}89 \\
(68 \%)\end{array}$ & $\begin{array}{l}26 \\
(29.21 \%)\end{array}$ & - & - & - & $58(65.17 \%)$ & $5(5.62 \%)$ & $\begin{array}{l}40 \\
(31 \%)\end{array}$ & $\begin{array}{l}22 \\
(55.00 \%)\end{array}$ & - & - \\
\hline Maucourant & 27 & $\begin{array}{l}10 \\
(37 \%)\end{array}$ & $\begin{array}{l}1 \\
(10.00 \%)\end{array}$ & $\begin{array}{l}2 \\
(20.00 \%)\end{array}$ & - & $\begin{array}{l}2 \\
(20.00 \%)\end{array}$ & - & $\begin{array}{l}5 \\
(50.00 \%)\end{array}$ & $\begin{array}{l}17 \\
(62 \%)\end{array}$ & $\begin{array}{l}2 \\
(11.76 \%)\end{array}$ & $\begin{array}{l}5 \\
(29.41 \%)\end{array}$ & - \\
\hline Xie & 619 & $\begin{array}{l}469 \\
(75 \%)\end{array}$ & - & - & 32 (6.82\%) & - & - & $\begin{array}{l}437 \\
(93.18 \%)\end{array}$ & $\begin{array}{l}150 \\
(24 \%)\end{array}$ & - & - & 1 s \\
\hline Fox & 55 & $\begin{array}{l}30 \\
(54 \%)\end{array}$ & $1(3.33 \%)$ & $\begin{array}{l}4 \\
(13.33 \%)\end{array}$ & - & $\begin{array}{l}17 \\
(56.67 \%)\end{array}$ & - & $\begin{array}{l}8 \\
(26.67 \%)\end{array}$ & $\begin{array}{l}25 \\
(45 \%)\end{array}$ & $0(0.00 \%)$ & $\begin{array}{l}2 \\
(8.00 \%)\end{array}$ & - \\
\hline Zhang, Cao & 240 & $\begin{array}{l}162 \\
(67 \%)\end{array}$ & $2(1.23 \%)$ & $6(3.70 \%)$ & - & - & - & $\begin{array}{l}154 \\
(95.06 \%)\end{array}$ & $\begin{array}{l}78 \\
(32 \%)\end{array}$ & $4(5.13 \%)$ & $\begin{array}{l}4 \\
(5.13 \%)\end{array}$ & - \\
\hline Kurashima & 53 & $\begin{array}{l}10 \\
(18 \%)\end{array}$ & - & - & $3(30.00 \%)$ & - & - & $\begin{array}{l}7 \\
(70.00 \%)\end{array}$ & $\begin{array}{l}43 \\
(81 \%)\end{array}$ & - & - & 24 \\
\hline Zhan & 75 & $\begin{array}{l}\text { NA } \\
\text { (NA\%) }\end{array}$ & - & - & - & - & - & - & $\begin{array}{l}75 \\
(100 \%)\end{array}$ & - & - & 9 \\
\hline Omrani & 858 & $\begin{array}{l}806 \\
(93 \%)\end{array}$ & - & - & $121(15.01 \%)$ & - & - & $\begin{array}{l}685 \\
(84.99 \%)\end{array}$ & $\begin{array}{l}52 \\
(6 \%)\end{array}$ & - & - & 9 \\
\hline Marcos & 918 & $\begin{array}{l}555 \\
(60 \%)\end{array}$ & $\begin{array}{l}38 \\
(6.85 \%)\end{array}$ & - & $69(12.43 \%)$ & - & - & $\begin{array}{l}448 \\
(80.72 \%)\end{array}$ & $\begin{array}{l}363 \\
(39 \%)\end{array}$ & $\begin{array}{l}18 \\
(4.96 \%)\end{array}$ & - & $7^{\circ}$ \\
\hline $\begin{array}{l}\text { Hoertel, } \\
\text { Sanchez- } \\
\text { Rico }\end{array}$ & 7345 & $\begin{array}{l}6014 \\
(81 \%)\end{array}$ & $\begin{array}{l}433 \\
(7.20 \%)\end{array}$ & - & - & - & - & $\begin{array}{l}5581 \\
(92.80 \%)\end{array}$ & $\begin{array}{l}1331 \\
(18 \%)\end{array}$ & $\begin{array}{l}190 \\
(14.27 \%)\end{array}$ & - & - \\
\hline Qi & 267 & $\begin{array}{l}217 \\
(81 \%)\end{array}$ & $\begin{array}{l}22 \\
(10.14 \%)\end{array}$ & - & - & - & 195 (89.86\%) & - & $\begin{array}{l}50 \\
(18 \%)\end{array}$ & $\begin{array}{l}31 \\
(62.00 \%)\end{array}$ & - & - \\
\hline Monteiro & 112 & $\begin{array}{l}84 \\
(75 \%)\end{array}$ & $3(3.57 \%)$ & $\begin{array}{l}14 \\
(16.67 \%)\end{array}$ & - & $\begin{array}{l}63 \\
(75.00 \%)\end{array}$ & - & $4(4.76 \%)$ & $\begin{array}{l}28 \\
(25 \%)\end{array}$ & $\begin{array}{l}4 \\
(14.29 \%)\end{array}$ & $\begin{array}{l}6 \\
(21.43 \%)\end{array}$ & - \\
\hline Dashti & 1381 & $\begin{array}{l}619 \\
(44 \%)\end{array}$ & - & - & 239 (38.61\%) & $\begin{array}{l}292 \\
(47.17 \%)\end{array}$ & - & $\begin{array}{l}88 \\
(14.22 \%)\end{array}$ & $\begin{array}{l}762 \\
(55 \%)\end{array}$ & - & - & $3 i$ \\
\hline Morshed & 103 & $\begin{array}{l}87 \\
(84 \%)\end{array}$ & $\begin{array}{l}28 \\
(32.18 \%)\end{array}$ & - & - & - & $59(67.82 \%)$ & - & $\begin{array}{l}16 \\
(15 \%)\end{array}$ & $\begin{array}{l}4 \\
(25.00 \%)\end{array}$ & - & - \\
\hline Zhou, Sun & 144 & $\begin{array}{l}108 \\
(75 \%)\end{array}$ & $\begin{array}{l}11 \\
(10.19 \%)\end{array}$ & - & - & - & - & $\begin{array}{l}97 \\
(89.81 \%)\end{array}$ & $\begin{array}{l}36 \\
(25 \%)\end{array}$ & $2(5.56 \%)$ & - & - \\
\hline $\begin{array}{l}\text { Hippisley- } \\
\text { Cox }\end{array}$ & - & NA & - & - & - & - & - & - & 1286 & $\begin{array}{l}56 \\
(4.35 \%)\end{array}$ & $\begin{array}{l}427 \\
(33.20 \%)\end{array}$ & - \\
\hline Zhao, Chen & 641 & $\begin{array}{l}398 \\
(62 \%)\end{array}$ & $\begin{array}{l}87 \\
(21.86 \%)\end{array}$ & - & - & - & - & $\begin{array}{l}311 \\
(78.14 \%)\end{array}$ & $\begin{array}{l}195 \\
(30 \%)\end{array}$ & $\begin{array}{l}52 \\
(26.67 \%)\end{array}$ & - & - \\
\hline Qu & 246 & $\begin{array}{l}226 \\
(91 \%)\end{array}$ & $\begin{array}{l}90 \\
(39.82 \%)\end{array}$ & - & - & - & - & $\begin{array}{l}136 \\
(60.18 \%)\end{array}$ & $\begin{array}{l}20 \\
(8 \%)\end{array}$ & $\begin{array}{l}14 \\
(70.00 \%)\end{array}$ & - & - \\
\hline Ren & 432 & $\begin{array}{l}314 \\
(72 \%)\end{array}$ & $\begin{array}{l}26 \\
(8.28 \%)\end{array}$ & - & - & $\begin{array}{l}288 \\
(91.72 \%)\end{array}$ & - & - & $\begin{array}{l}118 \\
(27 \%)\end{array}$ & $\begin{array}{l}17 \\
(14.41 \%)\end{array}$ & - & - \\
\hline Yan & 578 & $\begin{array}{l}450 \\
(77 \%)\end{array}$ & $\begin{array}{l}31 \\
(6.89 \%)\end{array}$ & - & - & - & - & $\begin{array}{l}419 \\
(93.11 \%)\end{array}$ & $\begin{array}{l}128 \\
(22 \%)\end{array}$ & $\begin{array}{l}20 \\
(15.62 \%)\end{array}$ & - & - \\
\hline Nicholson & 1042 & $\begin{array}{l}550 \\
(52 \%)\end{array}$ & $\begin{array}{l}37 \\
(6.73 \%)\end{array}$ & $\begin{array}{l}106 \\
(19.27 \%)\end{array}$ & - & $\begin{array}{l}211 \\
(38.36 \%)\end{array}$ & - & $\begin{array}{l}196 \\
(35.64 \%)\end{array}$ & $\begin{array}{l}401 \\
(38 \%)\end{array}$ & $\begin{array}{l}41 \\
(10.22 \%)\end{array}$ & $\begin{array}{l}92 \\
(22.94 \%)\end{array}$ & - \\
\hline Zhu & 432 & $\begin{array}{l}285 \\
(65 \%)\end{array}$ & $\begin{array}{l}46 \\
(16.14 \%)\end{array}$ & - & - & - & - & $\begin{array}{l}239 \\
(83.86 \%)\end{array}$ & $\begin{array}{l}147 \\
(34 \%)\end{array}$ & $\begin{array}{l}16 \\
(10.88 \%)\end{array}$ & - & - \\
\hline Kalan & 193 & $\begin{array}{l}122 \\
(63 \%)\end{array}$ & $9(7.38 \%)$ & - & - & $\begin{array}{l}102 \\
(83.61 \%)\end{array}$ & - & $\begin{array}{l}11 \\
(9.02 \%)\end{array}$ & $\begin{array}{l}71 \\
(36 \%)\end{array}$ & $5(7.04 \%)$ & - & - \\
\hline
\end{tabular}




\begin{tabular}{|c|c|c|c|c|c|c|c|c|c|c|c|c|}
\hline Burrell & 204 & $\begin{array}{l}85 \\
(41 \%)\end{array}$ & - & - & 7 (8.24\%) & - & 75 (88.24\%) & 3 (3.53\%) & $\begin{array}{l}11 y \\
(58 \%)\end{array}$ & - & - & 2( \\
\hline Chudasama & 1706 & $\begin{array}{l}\text { NA } \\
\text { (NA\%) }\end{array}$ & - & - & - & - & - & - & $\begin{array}{l}1706 \\
(100 \%)\end{array}$ & $\begin{array}{l}235 \\
(13.77 \%)\end{array}$ & $\begin{array}{l}699 \\
(40.97 \%)\end{array}$ & 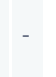 \\
\hline Lamure & 89 & $\begin{array}{l}\text { NA } \\
\text { (NA\%) }\end{array}$ & - & - & - & - & - & - & $\begin{array}{l}25 \\
(28 \%)\end{array}$ & $1(4.00 \%)$ & $\begin{array}{l}5 \\
(20.00 \%)\end{array}$ & 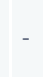 \\
\hline $\begin{array}{l}\text { Zhou, He, } \\
\text { Yang }\end{array}$ & 1,087 & $\begin{array}{l}990 \\
(91 \%)\end{array}$ & - & - & 849 (85.76\%) & $\begin{array}{l}141 \\
(14.24 \%)\end{array}$ & - & - & $\begin{array}{l}97 \\
(8 \%)\end{array}$ & - & - & $7 !$ \\
\hline Zhou, Qin & 51 & $\begin{array}{l}\text { NA } \\
\text { (NA\%) }\end{array}$ & - & - & - & - & - & - & $\begin{array}{l}51 \\
(100 \%)\end{array}$ & - & - & 4( \\
\hline Zhan, Liu & 405 & $\begin{array}{l}257 \\
(63 \%)\end{array}$ & - & - & 21 (8.17\%) & $\begin{array}{l}236 \\
(91.83 \%)\end{array}$ & - & - & $\begin{array}{l}148 \\
(36 \%)\end{array}$ & - & - & $2 !$ \\
\hline $\begin{array}{l}\text { Li, Long, } \\
\text { Zhang }\end{array}$ & 954 & $\begin{array}{l}838 \\
(87 \%)\end{array}$ & - & - & 34 (4.06\%) & $\begin{array}{l}804 \\
(95.94 \%)\end{array}$ & - & - & $\begin{array}{l}116 \\
(12 \%)\end{array}$ & - & - & $2 !$ \\
\hline Jakob & 2,155 & $\begin{array}{l}1400 \\
(64 \%)\end{array}$ & - & - & 92 (6.57\%) & $\begin{array}{l}99 \\
(7.07 \%)\end{array}$ & - & $\begin{array}{l}669 \\
(47.79 \%)\end{array}$ & $\begin{array}{l}755 \\
(35 \%)\end{array}$ & $\begin{array}{l}51 \\
(6.75 \%)\end{array}$ & $\begin{array}{l}58 \\
(7.68 \%)\end{array}$ & - \\
\hline Aksu & 123 & $\begin{array}{l}34 \\
(27 \%)\end{array}$ & 3 (8.82\%) & - & - & - & 31 (91.18\%) & - & $\begin{array}{l}89 \\
(72 \%)\end{array}$ & $\begin{array}{l}11 \\
(12.36 \%)\end{array}$ & - & - \\
\hline Adrish & 1,173 & $\begin{array}{l}162 \\
(13 \%)\end{array}$ & - & - & $36(22.22 \%)$ & - & 126 (77.78\%) & - & $\begin{array}{l}1011 \\
(86 \%)\end{array}$ & - & - & 36 \\
\hline $\begin{array}{l}\text { Hoertel, } \\
\text { Sanchez, } \\
\text { Vernet }\end{array}$ & 12,210 & $\begin{array}{l}11018 \\
(90 \%)\end{array}$ & $\begin{array}{l}921 \\
(8.36 \%)\end{array}$ & - & - & - & - & $\begin{array}{l}10097 \\
(91.64 \%)\end{array}$ & $\begin{array}{l}1192 \\
(9 \%)\end{array}$ & $\begin{array}{l}181 \\
(15.18 \%)\end{array}$ & - & - \\
\hline $\begin{array}{l}\text { Vila- } \\
\text { Corcoles, } \\
\text { Statue- } \\
\text { Gracia }\end{array}$ & 282 & $\begin{array}{l}218 \\
(77 \%)\end{array}$ & - & - & 21 (9.63\%) & - & - & $\begin{array}{l}197 \\
(90.37 \%)\end{array}$ & $\begin{array}{l}64 \\
(22 \%)\end{array}$ & - & - & 4 \\
\hline 4. & & & & & & & & & & & & - \\
\hline
\end{tabular}

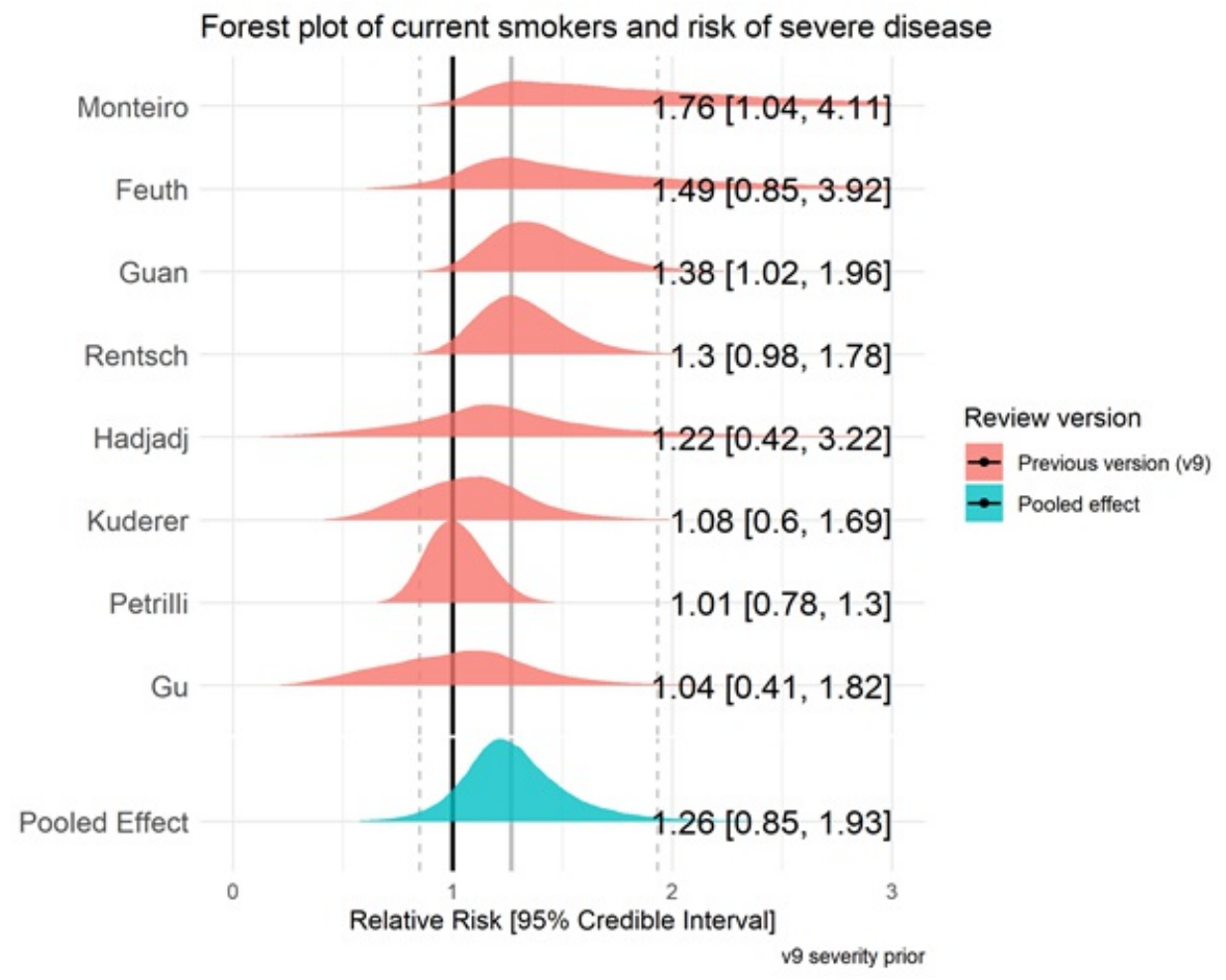

Figure 7. Forest plot for the risk of severe disease in current vs. never smokers. The prior from the 


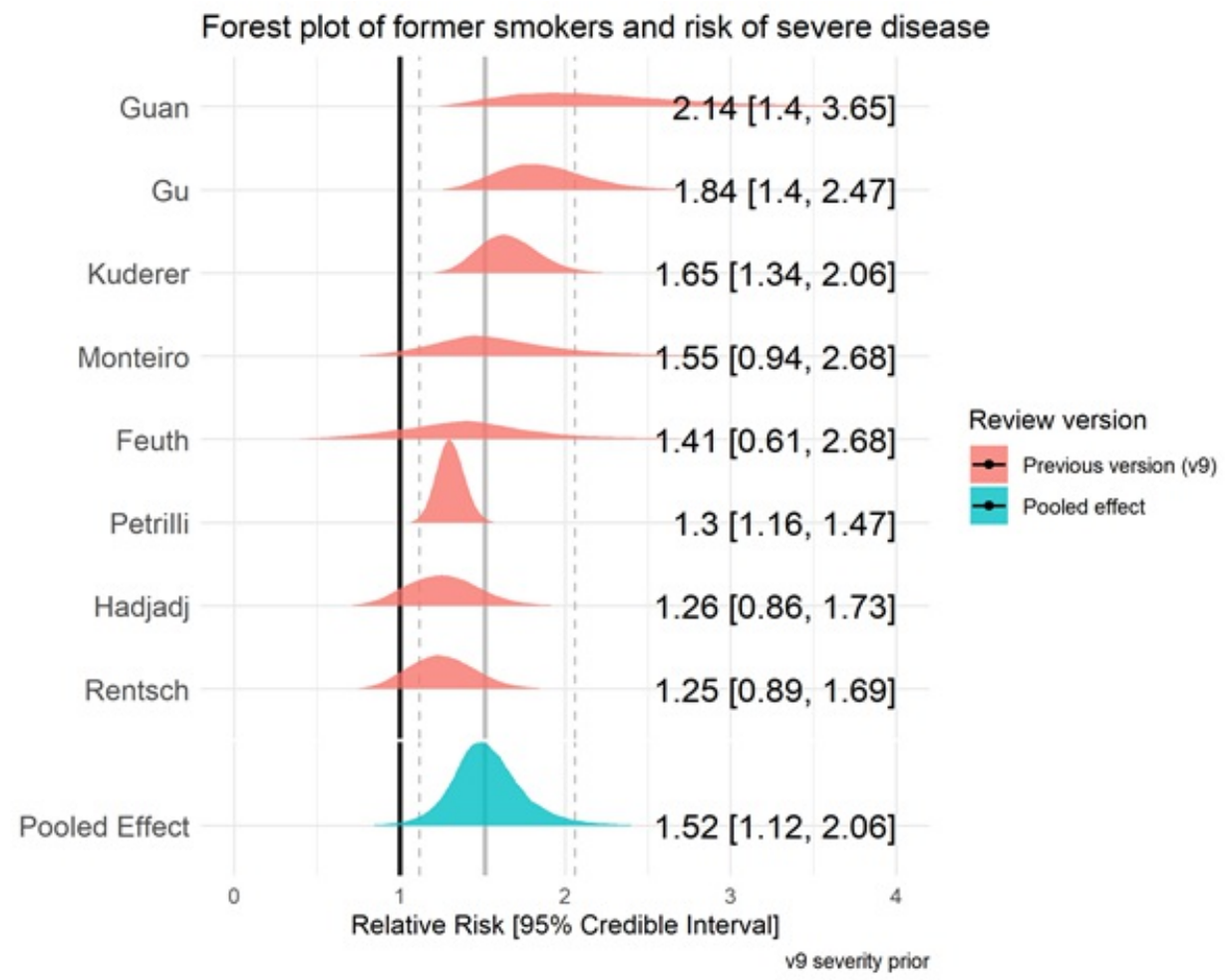

Figure 8. Forest plot for the risk of severe disease in former vs. never smokers. The prior from the previous review version (v9) was $\mathrm{RR}=1.52$.

\section{Mortality by smoking status}

Seventy-seven studies reported mortality from COVID-19 by smoking status (see Table 5), with 16 'fair' quality studies included in meta-analyses (see Figure 9 and 10). Current $(R R=1.05,95 \% \mathrm{Crl}=0.77-1.41, \tau=0.39)$ and former $(R R=$ $1.40,95 \% \mathrm{Crl}=1.2-1.64, \tau=0.19)$ compared with never smokers were at increased risk of in-hospital mortality from COVID-19. However, data for current smokers were inconclusive and favoured there being no important association. The probability of current and former smokers being at greater risk of in-hospital mortality $(R R \geq 1.1)$ compared with never smokers was $38 \%$ and $99.8 \%$, respectively. Results were materially unchanged in two sensitivity analyses.

Table 5. Mortality by smoking status.

\begin{tabular}{|c|c|c|c|c|c|c|c|c|c|c|c|c|}
\hline \multirow[b]{2}{*}{ Author } & \multirow[b]{2}{*}{$\begin{array}{l}\text { Population } \\
\text { with } \\
\text { mortality }\end{array}$} & \multicolumn{7}{|c|}{ Recovered } & \multicolumn{4}{|l|}{ Died } \\
\hline & & N (\%) & $\begin{array}{l}\text { Current } \\
\text { smoker } \\
(\%)\end{array}$ & $\begin{array}{l}\text { Former } \\
\text { smoker } \\
(\%)\end{array}$ & $\begin{array}{l}\text { Current/former } \\
\text { smoker (\%) }\end{array}$ & $\begin{array}{l}\text { Never } \\
\text { smoker } \\
(\%)\end{array}$ & $\begin{array}{l}\text { Never/unknown } \\
\text { smoker (\%) }\end{array}$ & $\begin{array}{l}\text { Not } \\
\text { stated } \\
(\%)\end{array}$ & N (\%) & $\begin{array}{l}\text { Current } \\
\text { smoker } \\
(\%)\end{array}$ & $\begin{array}{l}\text { Former } \\
\text { smoker } \\
(\%)\end{array}$ & $\begin{array}{l}\text { Curr } \\
\text { smol }\end{array}$ \\
\hline Chen & 274 & $\begin{array}{l}161 \\
(58 \%)\end{array}$ & $\begin{array}{l}5 \\
(3.11 \%)\end{array}$ & $\begin{array}{l}5 \\
(3.11 \%)\end{array}$ & - & - & - & $\begin{array}{l}151 \\
(93.79 \%)\end{array}$ & $\begin{array}{l}113 \\
(41 \%)\end{array}$ & $\begin{array}{l}7 \\
(6.19 \%)\end{array}$ & $\begin{array}{l}2 \\
(1.77 \%)\end{array}$ & - \\
\hline
\end{tabular}




\begin{tabular}{|c|c|c|c|c|c|c|c|c|c|c|c|c|}
\hline Zhou, Yu & 191 & $\begin{array}{l}137 \\
(71 \%)\end{array}$ & $\begin{array}{l}6 \\
(4.38 \%)\end{array}$ & - & - & - & - & $\begin{array}{l}131 \\
(95.62 \%)\end{array}$ & $\begin{array}{l}54 \\
(28 \%)\end{array}$ & $\begin{array}{l}5 \\
(9.26 \%)\end{array}$ & - & - \\
\hline Yang, Yu & 52 & $\begin{array}{l}20 \\
(38 \%)\end{array}$ & $\begin{array}{l}2 \\
(10.00 \%)\end{array}$ & - & - & - & $18(90.00 \%)$ & - & $\begin{array}{l}32 \\
(61 \%)\end{array}$ & - & - & - \\
\hline Borobia & 2226 & $\begin{array}{l}1766 \\
(79 \%)\end{array}$ & $\begin{array}{l}113 \\
(6.40 \%)\end{array}$ & - & - & - & - & $\begin{array}{l}1653 \\
(93.60 \%)\end{array}$ & $\begin{array}{l}460 \\
(20 \%)\end{array}$ & $\begin{array}{l}44 \\
(9.57 \%)\end{array}$ & - & - \\
\hline Giacomelli & 233 & $\begin{array}{l}185 \\
(79 \%)\end{array}$ & - & - & $53(28.65 \%)$ & $\begin{array}{l}132 \\
(71.35 \%)\end{array}$ & - & - & $\begin{array}{l}48 \\
(20 \%)\end{array}$ & - & - & $17(3$ \\
\hline Yao & 108 & $\begin{array}{l}96 \\
(88 \%)\end{array}$ & $\begin{array}{l}1 \\
(1.04 \%)\end{array}$ & - & - & - & - & $\begin{array}{l}95 \\
(98.96 \%)\end{array}$ & $\begin{array}{l}12 \\
(11 \%)\end{array}$ & $\begin{array}{l}3 \\
(25.00 \%)\end{array}$ & - & - \\
\hline Carillo-Vega & 9946 & $\begin{array}{l}8983 \\
(90 \%)\end{array}$ & $\begin{array}{l}795 \\
(8.85 \%)\end{array}$ & - & - & - & - & $\begin{array}{l}8188 \\
(91.15 \%)\end{array}$ & $\begin{array}{l}963 \\
(9 \%)\end{array}$ & $\begin{array}{l}99 \\
(10.28 \%)\end{array}$ & - & - \\
\hline Heng & 51 & $\begin{array}{l}39 \\
(76 \%)\end{array}$ & $\begin{array}{l}6 \\
(15.38 \%)\end{array}$ & - & - & - & - & $\begin{array}{l}33 \\
(84.62 \%)\end{array}$ & $\begin{array}{l}12 \\
(23 \%)\end{array}$ & $\begin{array}{l}1 \\
(8.33 \%)\end{array}$ & - & - \\
\hline Chen, Jiang & 135 & $\begin{array}{l}\text { NA } \\
\text { (NA\%) }\end{array}$ & - & - & - & - & - & - & $\begin{array}{l}31 \\
(22 \%)\end{array}$ & - & - & $4(12$ \\
\hline Heili-Frades & 4712 & $\begin{array}{l}4086 \\
(86 \%)\end{array}$ & $\begin{array}{l}210 \\
(5.14 \%)\end{array}$ & $\begin{array}{l}659 \\
(16.13 \%)\end{array}$ & - & - & 3217 (78.73\%) & - & $\begin{array}{l}626 \\
(13 \%)\end{array}$ & $\begin{array}{l}23 \\
(3.67 \%)\end{array}$ & $\begin{array}{l}161 \\
(25.72 \%)\end{array}$ & - \\
\hline Kim, Garg & 2490 & $\begin{array}{l}2070 \\
(83 \%)\end{array}$ & $\begin{array}{l}128 \\
(6.18 \%)\end{array}$ & $\begin{array}{l}481 \\
(23.24 \%)\end{array}$ & - & - & $1461(70.58 \%)$ & - & $\begin{array}{l}420 \\
(16 \%)\end{array}$ & $\begin{array}{l}22 \\
(5.24 \%)\end{array}$ & $\begin{array}{l}161 \\
(38.33 \%)\end{array}$ & - \\
\hline Al-Hindawi & 31 & $\begin{array}{l}15 \\
(48 \%)\end{array}$ & $\begin{array}{l}0 \\
(0.00 \%)\end{array}$ & $\begin{array}{l}10 \\
(66.67 \%)\end{array}$ & - & $\begin{array}{l}5 \\
(33.33 \%)\end{array}$ & - & - & $\begin{array}{l}16 \\
(51 \%)\end{array}$ & $\begin{array}{l}1 \\
(6.25 \%)\end{array}$ & $\begin{array}{l}12 \\
(75.00 \%)\end{array}$ & - \\
\hline Louis & 22 & $\begin{array}{l}16 \\
(72 \%)\end{array}$ & - & - & 7 (43.75\%) & - & - & $\begin{array}{l}9 \\
(56.25 \%)\end{array}$ & $\begin{array}{l}6 \\
(27 \%)\end{array}$ & - & - & $3(50$ \\
\hline Soto-Mota & 400 & $\begin{array}{l}200 \\
(50 \%)\end{array}$ & - & - & $23(11.50 \%)$ & - & - & $\begin{array}{l}177 \\
(88.50 \%)\end{array}$ & $\begin{array}{l}200 \\
(50 \%)\end{array}$ & - & - & $25(1$ \\
\hline Garibaldi & 747 & $\begin{array}{l}634 \\
(84 \%)\end{array}$ & $\begin{array}{l}36 \\
(5.68 \%)\end{array}$ & $\begin{array}{l}129 \\
(20.35 \%)\end{array}$ & - & - & - & $\begin{array}{l}469 \\
(73.97 \%)\end{array}$ & $\begin{array}{l}113 \\
(15 \%)\end{array}$ & $\begin{array}{l}6 \\
(5.31 \%)\end{array}$ & $\begin{array}{l}36 \\
(31.86 \%)\end{array}$ & - \\
\hline Docherty & 13364 & $\begin{array}{l}8199 \\
(61 \%)\end{array}$ & $\begin{array}{l}370 \\
(4.51 \%)\end{array}$ & $\begin{array}{l}1832 \\
(22.34 \%)\end{array}$ & - & $\begin{array}{l}4179 \\
(50.97 \%)\end{array}$ & - & $\begin{array}{l}1818 \\
(22.17 \%)\end{array}$ & $\begin{array}{l}5165 \\
(38 \%)\end{array}$ & $\begin{array}{l}214 \\
(4.14 \%)\end{array}$ & $\begin{array}{l}1350 \\
(26.14 \%)\end{array}$ & - \\
\hline Kuderer & 928 & $\begin{array}{l}807 \\
(86 \%)\end{array}$ & $\begin{array}{l}38 \\
(4.71 \%)\end{array}$ & $\begin{array}{l}262 \\
(32.47 \%)\end{array}$ & - & $\begin{array}{l}425 \\
(52.66 \%)\end{array}$ & - & $\begin{array}{l}31 \\
(3.84 \%)\end{array}$ & $\begin{array}{l}121 \\
(13 \%)\end{array}$ & $\begin{array}{l}5 \\
(4.13 \%)\end{array}$ & $\begin{array}{l}64 \\
(52.89 \%)\end{array}$ & - \\
\hline Ramlall & 11116 & $\begin{array}{l}10498 \\
(94 \%)\end{array}$ & - & - & $2771(26.40 \%)$ & $\begin{array}{l}7727 \\
(73.60 \%)\end{array}$ & - & - & $\begin{array}{l}618 \\
(5 \%)\end{array}$ & - & - & 2081 \\
\hline $\begin{array}{l}\text { Wang, } \\
\text { Oekelen }\end{array}$ & 57 & $\begin{array}{l}43 \\
(75 \%)\end{array}$ & - & - & $14(32.56 \%)$ & - & - & $\begin{array}{l}29 \\
(67.44 \%)\end{array}$ & $\begin{array}{l}14 \\
(24 \%)\end{array}$ & - & - & $7(50$ \\
\hline $\begin{array}{l}\text { Martinez- } \\
\text { Portilla }\end{array}$ & 224 & $\begin{array}{l}217 \\
(96 \%)\end{array}$ & - & - & 7 (3.23\%) & - & - & $\begin{array}{l}210 \\
(96.77 \%)\end{array}$ & 7 (3\%) & - & - & $0(0.1$ \\
\hline Cen & 1007 & $\begin{array}{l}964 \\
(95 \%)\end{array}$ & - & - & 87 (9.02\%) & - & - & $\begin{array}{l}877 \\
(90.98 \%)\end{array}$ & $\begin{array}{l}43 \\
(4 \%)\end{array}$ & - & - & $1(2 .:$ \\
\hline Klang & 3406 & $\begin{array}{l}2270 \\
(66 \%)\end{array}$ & - & - & 492 (21.67\%) & - & - & $\begin{array}{l}1778 \\
(78.33 \%)\end{array}$ & $\begin{array}{l}1136 \\
(33 \%)\end{array}$ & - & - & 3011 \\
\hline Wang, Zhong & 5510 & $\begin{array}{l}4874 \\
(88 \%)\end{array}$ & $\begin{array}{l}247 \\
(5.07 \%)\end{array}$ & $\begin{array}{l}1083 \\
(22.22 \%)\end{array}$ & - & $\begin{array}{l}3544 \\
(72.71 \%)\end{array}$ & - & - & $\begin{array}{l}636 \\
(11 \%)\end{array}$ & $\begin{array}{l}28 \\
(4.40 \%)\end{array}$ & $\begin{array}{l}214 \\
(33.65 \%)\end{array}$ & - \\
\hline Miyara & 338 & $\begin{array}{l}211 \\
(62 \%)\end{array}$ & $\begin{array}{l}13 \\
(6.16 \%)\end{array}$ & $\begin{array}{l}58 \\
(27.49 \%)\end{array}$ & - & $\begin{array}{l}141 \\
(66.82 \%)\end{array}$ & - & - & $\begin{array}{l}46 \\
(13 \%)\end{array}$ & $\begin{array}{l}1 \\
(2.17 \%)\end{array}$ & $\begin{array}{l}23 \\
(50.00 \%)\end{array}$ & - \\
\hline Rajter & 255 & $\begin{array}{l}209 \\
(81 \%)\end{array}$ & - & - & $28(13.40 \%)$ & $\begin{array}{l}181 \\
(86.60 \%)\end{array}$ & - & - & $\begin{array}{l}53 \\
(20 \%)\end{array}$ & - & - & $18(3$ \\
\hline Zeng & 1031 & $\begin{array}{l}866 \\
(84 \%)\end{array}$ & - & - & $69(7.97 \%)$ & - & - & $\begin{array}{l}797 \\
(92.03 \%)\end{array}$ & $\begin{array}{l}165 \\
(16 \%)\end{array}$ & - & - & $36(2$ \\
\hline
\end{tabular}




\begin{tabular}{|c|c|c|c|c|c|c|c|c|c|c|c|c|}
\hline Chen, Yu & 1859 & $\begin{array}{l}1651 \\
(88 \%)\end{array}$ & $\begin{array}{l}32 \\
(1.94 \%)\end{array}$ & $\begin{array}{l}54 \\
(3.27 \%)\end{array}$ & - & $\begin{array}{l}1565 \\
(94.79 \%)\end{array}$ & - & - & $\begin{array}{l}208 \\
(11 \%)\end{array}$ & $\begin{array}{l}13 \\
(6.25 \%)\end{array}$ & $\begin{array}{l}12 \\
(5.77 \%)\end{array}$ & - \\
\hline Garassino & 190 & $\begin{array}{l}124 \\
(65 \%)\end{array}$ & - & - & $92(74.19 \%)$ & $\begin{array}{l}32 \\
(25.81 \%)\end{array}$ & - & - & $\begin{array}{l}66 \\
(34 \%)\end{array}$ & - & $\begin{array}{l}61 \\
(92.42 \%)\end{array}$ & - \\
\hline $\mathrm{Gu}$ & 884 & $\begin{array}{l}864 \\
(97 \%)\end{array}$ & $\begin{array}{l}40 \\
(4.63 \%)\end{array}$ & $\begin{array}{l}250 \\
(28.94 \%)\end{array}$ & - & $\begin{array}{l}219 \\
(25.35 \%)\end{array}$ & - & - & $\begin{array}{l}20 \\
(2 \%)\end{array}$ & $\begin{array}{l}0 \\
(0.00 \%)\end{array}$ & $\begin{array}{l}14 \\
(70.00 \%)\end{array}$ & - \\
\hline Nguyen & 356 & $\begin{array}{l}308 \\
(86 \%)\end{array}$ & - & - & $91(29.55 \%)$ & - & - & $\begin{array}{l}217 \\
(70.45 \%)\end{array}$ & $\begin{array}{l}45 \\
(12 \%)\end{array}$ & - & - & $23(5$ \\
\hline de Souza & 8443 & $\begin{array}{l}7826 \\
(92 \%)\end{array}$ & - & - & $95(1.21 \%)$ & - & $7571(96.74 \%)$ & $\begin{array}{l}160 \\
(2.04 \%)\end{array}$ & $\begin{array}{l}617 \\
(7 \%)\end{array}$ & - & - & $47(7$ \\
\hline $\begin{array}{l}\text { Shi, } \\
\text { Resurreccion }\end{array}$ & 256 & $\begin{array}{l}210 \\
(82 \%)\end{array}$ & - & - & $128(60.95 \%)$ & - & - & $\begin{array}{l}82 \\
(39.05 \%)\end{array}$ & $\begin{array}{l}46 \\
(17 \%)\end{array}$ & - & - & $26(5$ \\
\hline Xie & 619 & $\begin{array}{l}591 \\
(95 \%)\end{array}$ & - & - & $43(7.28 \%)$ & - & - & $\begin{array}{l}548 \\
(92.72 \%)\end{array}$ & $\begin{array}{l}28 \\
(4 \%)\end{array}$ & - & - & $8(28$ \\
\hline Fox & 54 & $\begin{array}{l}35 \\
(64 \%)\end{array}$ & $\begin{array}{l}1 \\
(2.86 \%)\end{array}$ & $\begin{array}{l}4 \\
(11.43 \%)\end{array}$ & - & $\begin{array}{l}18 \\
(51.43 \%)\end{array}$ & - & $\begin{array}{l}12 \\
(34.29 \%)\end{array}$ & $\begin{array}{l}19 \\
(35 \%)\end{array}$ & $\begin{array}{l}0 \\
(0.00 \%)\end{array}$ & $\begin{array}{l}2 \\
(10.53 \%)\end{array}$ & - \\
\hline Zhang, Cao & 289 & $\begin{array}{l}240 \\
(83 \%)\end{array}$ & $\begin{array}{l}10 \\
(4.17 \%)\end{array}$ & $\begin{array}{l}6 \\
(2.50 \%)\end{array}$ & - & - & - & $\begin{array}{l}224 \\
(93.33 \%)\end{array}$ & $\begin{array}{l}49 \\
(16 \%)\end{array}$ & $\begin{array}{l}4 \\
(8.16 \%)\end{array}$ & $\begin{array}{l}8 \\
(16.33 \%)\end{array}$ & - \\
\hline Gupta & 496 & $\begin{array}{l}255 \\
(51 \%)\end{array}$ & - & - & $15(5.88 \%)$ & - & 80 (31.37\%) & $\begin{array}{l}160 \\
(62.75 \%)\end{array}$ & $\begin{array}{l}241 \\
(48 \%)\end{array}$ & - & - & $21(8$ \\
\hline Thompson & 470 & $\begin{array}{l}301 \\
(64 \%)\end{array}$ & $\begin{array}{l}39 \\
(12.96 \%)\end{array}$ & $\begin{array}{l}79 \\
(26.25 \%)\end{array}$ & - & $\begin{array}{l}183 \\
(60.80 \%)\end{array}$ & - & - & $\begin{array}{l}169 \\
(35 \%)\end{array}$ & $\begin{array}{l}27 \\
(15.98 \%)\end{array}$ & $\begin{array}{l}49 \\
(28.99 \%)\end{array}$ & - \\
\hline Bernaola & 1645 & $\begin{array}{l}1382 \\
(84 \%)\end{array}$ & $\begin{array}{l}35 \\
(2.53 \%)\end{array}$ & $\begin{array}{l}146 \\
(10.56 \%)\end{array}$ & - & $\begin{array}{l}1201 \\
(86.90 \%)\end{array}$ & - & - & $\begin{array}{l}263 \\
(15 \%)\end{array}$ & $\begin{array}{l}6 \\
(2.28 \%)\end{array}$ & $\begin{array}{l}33 \\
(12.55 \%)\end{array}$ & - \\
\hline Islam & 654 & $\begin{array}{l}631 \\
(96 \%)\end{array}$ & $\begin{array}{l}103 \\
(16.32 \%)\end{array}$ & - & - & - & - & $\begin{array}{l}507 \\
(80.35 \%)\end{array}$ & $\begin{array}{l}23 \\
(3 \%)\end{array}$ & $\begin{array}{l}3 \\
(13.04 \%)\end{array}$ & - & - \\
\hline Philipose & 466 & $\begin{array}{l}267 \\
(57 \%)\end{array}$ & $\begin{array}{l}19 \\
(7.12 \%)\end{array}$ & $\begin{array}{l}204 \\
(76.40 \%)\end{array}$ & - & $\begin{array}{l}44 \\
(16.48 \%)\end{array}$ & - & - & $\begin{array}{l}199 \\
(42 \%)\end{array}$ & $\begin{array}{l}9 \\
(4.52 \%)\end{array}$ & $\begin{array}{l}137 \\
(68.84 \%)\end{array}$ & - \\
\hline Dashti & 4140 & $\begin{array}{l}3953 \\
(95 \%)\end{array}$ & - & - & $1068(27.02 \%)$ & $\begin{array}{l}2078 \\
(52.57 \%)\end{array}$ & - & $\begin{array}{l}804 \\
(20.34 \%)\end{array}$ & $\begin{array}{l}187 \\
(4 \%)\end{array}$ & - & - & 109 \\
\hline Fillmore & 1794 & $\begin{array}{l}1566 \\
(87 \%)\end{array}$ & $\begin{array}{l}408 \\
(26.05 \%)\end{array}$ & $\begin{array}{l}758 \\
(48.40 \%)\end{array}$ & - & $\begin{array}{l}279 \\
(17.82 \%)\end{array}$ & - & $\begin{array}{l}98 \\
(6.26 \%)\end{array}$ & $\begin{array}{l}228 \\
(12 \%)\end{array}$ & $\begin{array}{l}44 \\
(19.30 \%)\end{array}$ & $\begin{array}{l}141 \\
(61.84 \%)\end{array}$ & - \\
\hline Pan & 3536 & $\begin{array}{l}3302 \\
(93 \%)\end{array}$ & - & - & $862(26.11 \%)$ & - & - & $\begin{array}{l}2440 \\
(73.89 \%)\end{array}$ & $\begin{array}{l}234 \\
(6 \%)\end{array}$ & - & - & $82(3$ \\
\hline Zhao, Chen & 474 & $\begin{array}{l}398 \\
(83 \%)\end{array}$ & $\begin{array}{l}87 \\
(21.86 \%)\end{array}$ & - & - & - & - & $\begin{array}{l}311 \\
(78.14 \%)\end{array}$ & $\begin{array}{l}82 \\
(17 \%)\end{array}$ & $\begin{array}{l}36 \\
(43.90 \%)\end{array}$ & - & - \\
\hline Holman & 10989 & $\begin{array}{l}\text { NA } \\
\text { (NA\%) }\end{array}$ & - & - & - & - & - & - & $\begin{array}{l}10989 \\
(100 \%)\end{array}$ & $\begin{array}{l}609 \\
(5.54 \%)\end{array}$ & $\begin{array}{l}4684 \\
(42.62 \%)\end{array}$ & - \\
\hline Chand & 300 & $\begin{array}{l}143 \\
(47 \%)\end{array}$ & $\begin{array}{l}23 \\
(16.08 \%)\end{array}$ & - & - & - & - & $\begin{array}{l}120 \\
(83.92 \%)\end{array}$ & $\begin{array}{l}157 \\
(52 \%)\end{array}$ & $\begin{array}{l}44 \\
(28.03 \%)\end{array}$ & - & - \\
\hline Oliveira & 131 & $\begin{array}{l}105 \\
(80 \%)\end{array}$ & - & - & $16(15.24 \%)$ & - & $83(79.05 \%)$ & $6(5.71 \%)$ & $\begin{array}{l}26 \\
(19 \%)\end{array}$ & - & - & $7(26$ \\
\hline
\end{tabular}




\begin{tabular}{|c|c|c|c|c|c|c|c|c|c|c|c|c|}
\hline Inaila-Ivava & +1 & $(88 \%)$ & $(7.30 \%)$ & - & - & - & - & $(92.70 \%)$ & $(11 \%)$ & (8.21\%) & - & \\
\hline Rubio-Rivas & 186 & $\begin{array}{l}147 \\
(79 \%)\end{array}$ & $\begin{array}{l}7 \\
(4.76 \%)\end{array}$ & $\begin{array}{l}32 \\
(21.77 \%)\end{array}$ & - & $\begin{array}{l}108 \\
(73.47 \%)\end{array}$ & - & - & $\begin{array}{l}39 \\
(20 \%)\end{array}$ & $\begin{array}{l}1 \\
(2.56 \%)\end{array}$ & $\begin{array}{l}6 \\
(15.38 \%)\end{array}$ & - \\
\hline Ren & 432 & $\begin{array}{l}289 \\
(66 \%)\end{array}$ & $\begin{array}{l}25 \\
(8.65 \%)\end{array}$ & - & - & $\begin{array}{l}264 \\
(91.35 \%)\end{array}$ & - & - & $\begin{array}{l}143 \\
(33 \%)\end{array}$ & $\begin{array}{l}18 \\
(12.59 \%)\end{array}$ & - & 125 \\
\hline Ullah & 212 & $\begin{array}{l}158 \\
(74 \%)\end{array}$ & $\begin{array}{l}22 \\
(13.92 \%)\end{array}$ & $\begin{array}{l}67 \\
(42.41 \%)\end{array}$ & - & $\begin{array}{l}63 \\
(39.87 \%)\end{array}$ & - & $6(3.80 \%)$ & $\begin{array}{l}54 \\
(25 \%)\end{array}$ & $\begin{array}{l}2 \\
(3.70 \%)\end{array}$ & $\begin{array}{l}35 \\
(64.81 \%)\end{array}$ & - \\
\hline Dashti & 3401 & $\begin{array}{l}2892 \\
(85 \%)\end{array}$ & $\begin{array}{l}190 \\
(6.57 \%)\end{array}$ & $\begin{array}{l}689 \\
(23.82 \%)\end{array}$ & - & $\begin{array}{l}1756 \\
(60.72 \%)\end{array}$ & - & $\begin{array}{l}257 \\
(8.89 \%)\end{array}$ & $\begin{array}{l}509 \\
(14 \%)\end{array}$ & $\begin{array}{l}20 \\
(3.93 \%)\end{array}$ & $\begin{array}{l}171 \\
(33.60 \%)\end{array}$ & - \\
\hline Nicholson & 1040 & $\begin{array}{l}829 \\
(79 \%)\end{array}$ & $\begin{array}{l}70 \\
(8.44 \%)\end{array}$ & $\begin{array}{l}163 \\
(19.66 \%)\end{array}$ & - & $\begin{array}{l}320 \\
(38.60 \%)\end{array}$ & - & $\begin{array}{l}276 \\
(33.29 \%)\end{array}$ & $\begin{array}{l}211 \\
(20 \%)\end{array}$ & $\begin{array}{l}16 \\
(7.58 \%)\end{array}$ & $\begin{array}{l}68 \\
(32.23 \%)\end{array}$ & - \\
\hline Kalan & 193 & $\begin{array}{l}188 \\
(97 \%)\end{array}$ & $\begin{array}{l}14 \\
(7.45 \%)\end{array}$ & - & - & $\begin{array}{l}162 \\
(86.17 \%)\end{array}$ & - & $\begin{array}{l}12 \\
(6.38 \%)\end{array}$ & $5(2 \%)$ & $\begin{array}{l}0 \\
(0.00 \%)\end{array}$ & - & - \\
\hline Incerti & 13658 & $\begin{array}{l}11495 \\
(84 \%)\end{array}$ & $\begin{array}{l}785 \\
(6.83 \%)\end{array}$ & $\begin{array}{l}2450 \\
(21.31 \%)\end{array}$ & - & $\begin{array}{l}5450 \\
(47.41 \%)\end{array}$ & $2810(24.45 \%)$ & - & $\begin{array}{l}2163 \\
(15 \%)\end{array}$ & $\begin{array}{l}81 \\
(3.74 \%)\end{array}$ & $\begin{array}{l}642 \\
(29.68 \%)\end{array}$ & - \\
\hline loannou & 10131 & $\begin{array}{l}9033 \\
(89 \%)\end{array}$ & $\begin{array}{l}1054 \\
(11.67 \%)\end{array}$ & $\begin{array}{l}3549 \\
(39.29 \%)\end{array}$ & - & $\begin{array}{l}3339 \\
(36.96 \%)\end{array}$ & - & $\begin{array}{l}1091 \\
(12.08 \%)\end{array}$ & $\begin{array}{l}1098 \\
(10 \%)\end{array}$ & $\begin{array}{l}81 \\
(7.38 \%)\end{array}$ & $\begin{array}{l}528 \\
(48.09 \%)\end{array}$ & - \\
\hline Lamure & 89 & $\begin{array}{l}59 \\
(66 \%)\end{array}$ & $\begin{array}{l}4 \\
(6.78 \%)\end{array}$ & $\begin{array}{l}16 \\
(27.12 \%)\end{array}$ & - & $\begin{array}{l}31 \\
(52.54 \%)\end{array}$ & - & $\begin{array}{l}8 \\
(13.56 \%)\end{array}$ & $\begin{array}{l}30 \\
(33 \%)\end{array}$ & $\begin{array}{l}1 \\
(3.33 \%)\end{array}$ & $\begin{array}{l}13 \\
(43.33 \%)\end{array}$ & - \\
\hline Yadaw & 5051 & $\begin{array}{l}4635 \\
(91 \%)\end{array}$ & $\begin{array}{l}162 \\
(3.50 \%)\end{array}$ & $\begin{array}{l}709 \\
(15.30 \%)\end{array}$ & - & $\begin{array}{l}2394 \\
(51.65 \%)\end{array}$ & - & $\begin{array}{l}1370 \\
(29.56 \%)\end{array}$ & $\begin{array}{l}416 \\
(8 \%)\end{array}$ & $\begin{array}{l}17 \\
(4.09 \%)\end{array}$ & $\begin{array}{l}105 \\
(25.24 \%)\end{array}$ & - \\
\hline Zinellu & 105 & $\begin{array}{l}77 \\
(73 \%)\end{array}$ & $\begin{array}{l}24 \\
(31.17 \%)\end{array}$ & $\begin{array}{l}8 \\
(10.39 \%)\end{array}$ & - & $\begin{array}{l}45 \\
(58.44 \%)\end{array}$ & - & - & $\begin{array}{l}28 \\
(26 \%)\end{array}$ & $\begin{array}{l}8 \\
(28.57 \%)\end{array}$ & $\begin{array}{l}3 \\
(10.71 \%)\end{array}$ & - \\
\hline Zhang, Li & 399 & $\begin{array}{l}\text { NA } \\
\text { (NA\%) }\end{array}$ & - & - & - & - & - & - & $\begin{array}{l}399 \\
(100 \%)\end{array}$ & $\begin{array}{l}60 \\
(15.04 \%)\end{array}$ & $\begin{array}{l}186 \\
(46.62 \%)\end{array}$ & - \\
\hline $\begin{array}{l}\text { Wang, } \\
\text { Zheutlin }\end{array}$ & 2,448 & $\begin{array}{l}1706 \\
(69 \%)\end{array}$ & $\begin{array}{l}57 \\
(3.34 \%)\end{array}$ & $\begin{array}{l}315 \\
(18.46 \%)\end{array}$ & - & $\begin{array}{l}954 \\
(55.92 \%)\end{array}$ & - & $\begin{array}{l}380 \\
(22.27 \%)\end{array}$ & $\begin{array}{l}742 \\
(30 \%)\end{array}$ & $\begin{array}{l}25 \\
(3.37 \%)\end{array}$ & $\begin{array}{l}197 \\
(26.55 \%)\end{array}$ & - \\
\hline Torres-Macho & 1,968 & $\begin{array}{l}1643 \\
(83 \%)\end{array}$ & - & - & 335 (20.39\%) & - & - & $\begin{array}{l}1308 \\
(79.61 \%)\end{array}$ & $\begin{array}{l}325 \\
(16 \%)\end{array}$ & - & - & 125 \\
\hline Raines & 440 & $\begin{array}{l}408 \\
(92 \%)\end{array}$ & - & - & $222(54.41 \%)$ & $\begin{array}{l}186 \\
(45.59 \%)\end{array}$ & - & - & $\begin{array}{l}32 \\
(7 \%)\end{array}$ & - & - & $28(8$ \\
\hline $\begin{array}{l}\text { Parra- } \\
\text { Bracamonte }\end{array}$ & 331,298 & $\begin{array}{l}292988 \\
(88 \%)\end{array}$ & - & - & 21269 (7.26\%) & - & - & $\begin{array}{l}271719 \\
(92.74 \%)\end{array}$ & $\begin{array}{l}38310 \\
(11 \%)\end{array}$ & - & - & 3215 \\
\hline $\begin{array}{l}\text { Li, Long, } \\
\text { Zhang }\end{array}$ & 954 & $\begin{array}{l}876 \\
(91 \%)\end{array}$ & - & - & $48(5.48 \%)$ & $\begin{array}{l}828 \\
(94.52 \%)\end{array}$ & - & - & $\begin{array}{l}78 \\
(8 \%)\end{array}$ & - & - & $8(10$ \\
\hline Bellan & 407 & $\begin{array}{l}285 \\
(70 \%)\end{array}$ & $\begin{array}{l}30 \\
(10.53 \%)\end{array}$ & - & - & - & $191(67.02 \%)$ & $\begin{array}{l}64 \\
(22.46 \%)\end{array}$ & $\begin{array}{l}122 \\
(29 \%)\end{array}$ & $\begin{array}{l}24 \\
(19.67 \%)\end{array}$ & - & - \\
\hline Alharthy & 352 & $\begin{array}{l}239 \\
(67 \%)\end{array}$ & $\begin{array}{l}109 \\
(45.61 \%)\end{array}$ & - & - & - & $130(54.39 \%)$ & - & $\begin{array}{l}113 \\
(32 \%)\end{array}$ & $\begin{array}{l}65 \\
(57.52 \%)\end{array}$ & - & - \\
\hline Adrish & 1,173 & $\begin{array}{l}783 \\
(66 \%)\end{array}$ & - & - & $205(26.18 \%)$ & $\begin{array}{l}578 \\
(73.82 \%)\end{array}$ & - & - & $\begin{array}{l}390 \\
(33 \%)\end{array}$ & - & - & 1311 \\
\hline $\begin{array}{l}\text { Hoertel, } \\
\text { Sanchez, } \\
\text { Vernet }\end{array}$ & 12,210 & $\begin{array}{l}12210 \\
(100 \%)\end{array}$ & $\begin{array}{l}898 \\
(7.35 \%)\end{array}$ & - & - & - & - & $\begin{array}{l}9904 \\
(81.11 \%)\end{array}$ & $\begin{array}{l}1408 \\
(11 \%)\end{array}$ & $\begin{array}{l}204 \\
(14.49 \%)\end{array}$ & - & - \\
\hline Sourij & 238 & $\begin{array}{l}180 \\
(75 \%)\end{array}$ & $\begin{array}{l}4 \\
(2.22 \%)\end{array}$ & $\begin{array}{l}26 \\
(14.44 \%)\end{array}$ & - & $\begin{array}{l}150 \\
(83.33 \%)\end{array}$ & - & - & $\begin{array}{l}58 \\
(24 \%)\end{array}$ & $\begin{array}{l}0 \\
(0.00 \%)\end{array}$ & $\begin{array}{l}12 \\
(20.69 \%)\end{array}$ & - \\
\hline
\end{tabular}



Bisso
168
$(72 \%)$
$(10.66 \%)$
$(89.34 \%)$
$(27 \%)$
$(10.87 \%)$

Note. Solis et al. and the OpenSAFELY Collaborative reported on mortality by smoking status in a multivariable analysis but did not present raw data for both the exposure and outcome variables.

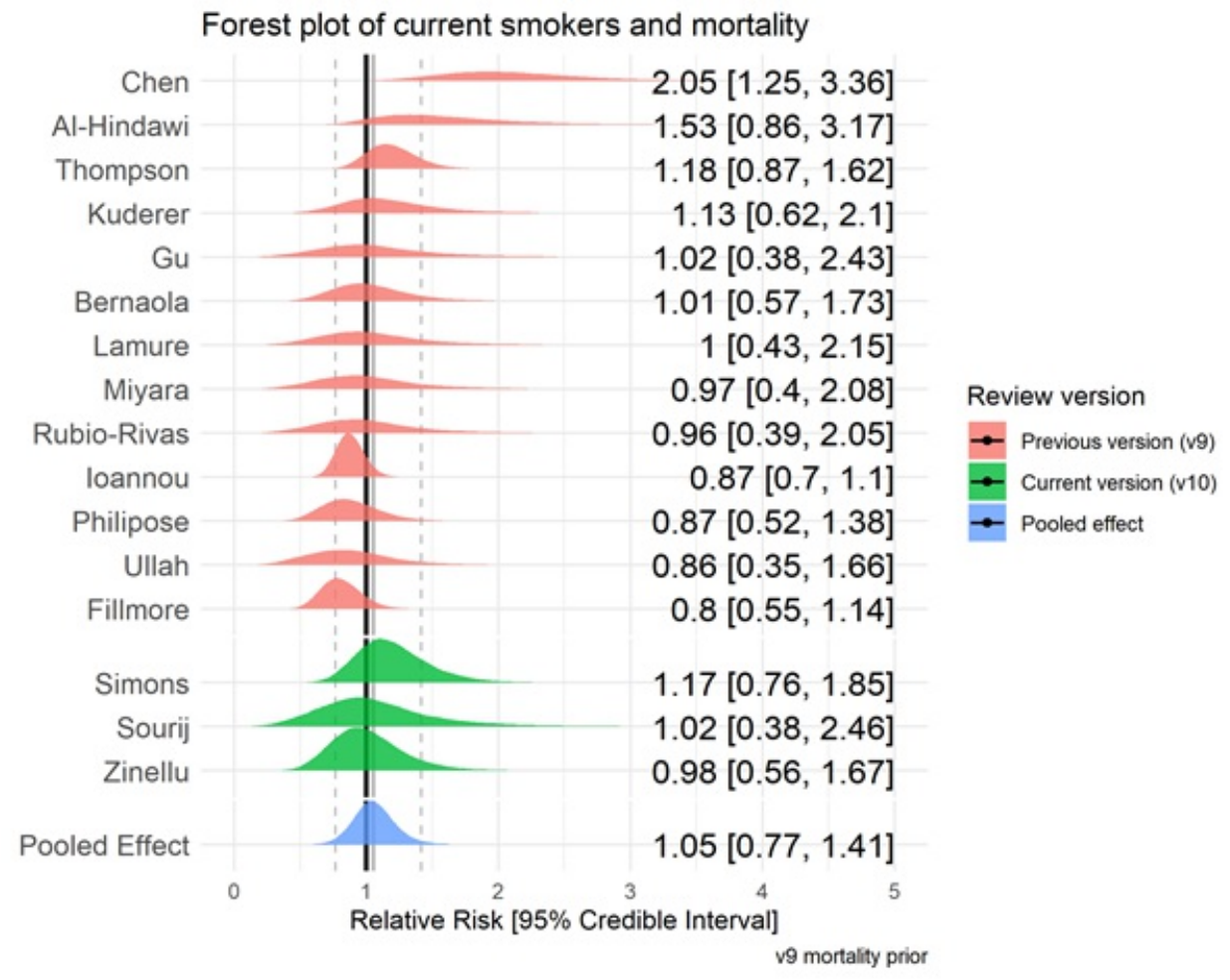

Figure 9. Forest plot for the risk of mortality in current vs. never smokers. The prior from the previous review version (v9) was $\mathrm{RR}=1.05$. 


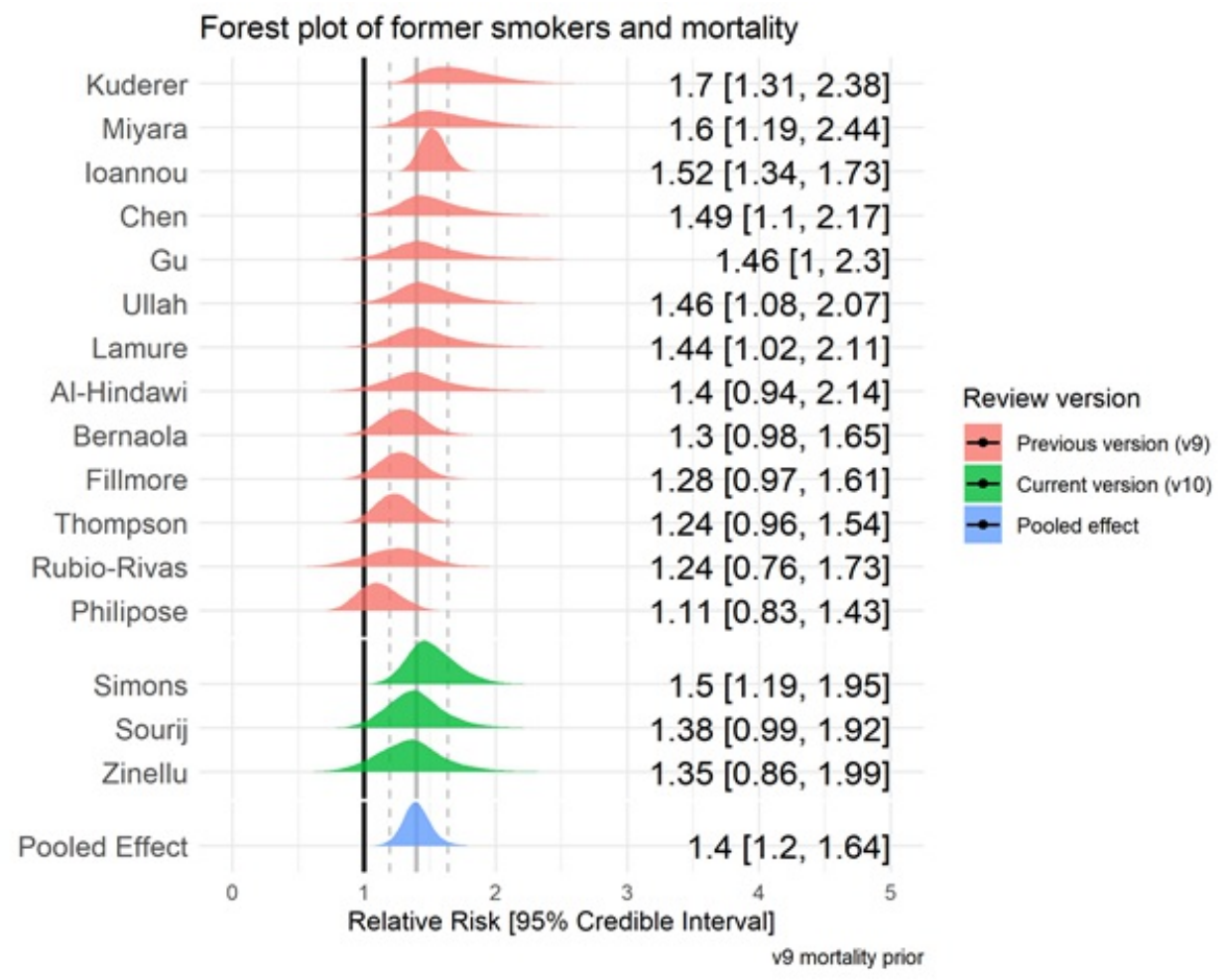

Figure 10. Forest plot for the risk of mortality in former smokers vs. never smokers. The prior from the previous version (v9) was $R R=1.39$.

\section{Discussion}

This living rapid review found uncertainty in the majority of 345 studies arising from the recording of smoking status.

Notwithstanding these uncertainties, compared with overall adult national prevalence estimates, recorded current smoking rates in most studies were lower than expected. In a subset of better-quality studies ( $n=25)$, current but not former smokers had a reduced risk of testing positive for SARS-CoV-2 but current smokers appeared somewhat more likely to present for testing and/or receive a test. Data for current smokers on the risk of hospitalisation, disease severity and mortality were inconclusive, and favoured there being no important associations with hospitalisation and mortality and a small but important increase in the risk of severe disease. Former smokers were at increased risk of hospitalisation, disease severity and mortality compared with never smokers.

\section{Issues complicating interpretation}

Interpretation of results from studies conducted during the first phase of the SARS-CoV-2 pandemic is complicated by several factors (see Figure 11):

\section{1) Exposure to SARS-CoV-2}


1. Exposure to the SARS-CoV-2 virus is heterogeneous with different subgroups at heightened risk of infection at different stages of the pandemic, at least partly due to differential contact matrices by age, sex and socioeconomic position ${ }^{36}$, which are associated with smoking status.

2. The probability of viral exposure depends largely on local prevalence, which varies over time. This likely introduces bias in studies assessing the rate of infection by smoking status conducted in the early phase of the pandemic.

\section{2) Infection with SARS-CoV-2}

1. Infection following viral exposure depends on individual differences in, for example, genetic susceptibility or immunocompetence, which are poorly understood at present. For example, the household secondary attack rate for COVID-19 is estimated at $17 \%{ }^{37}$.

2. Heated and humidified air may act to disrupt the ability of the virus to persist in the airway mucosa of smokers. There is some evidence that transient localised hyperthermia can inhibit replication of rhinoviruses, a non-enveloped virus that causes the common cold ${ }^{38}$. However, as SARS-CoV-2 is an enveloped virus ${ }^{39}$, it is unclear whether a similar protective effect against viral replication or invasion by heated and humidified air may occur.

\section{3) Symptomatic COVID-19}

1. An estimated $20 \%(95 \% \mathrm{Cl}=17-25 \%)$ of $\mathrm{COVID}-19$ cases are asymptomatic ${ }^{40}$, with some evidence suggesting younger people are more likely to be asymptomatic ${ }^{41}$. Testing is hence likely limited in some subgroups, with the potential for these groups to include an overrepresentation of current smokers.

2. Current and former smokers may be more likely to meet local criteria for community testing due to increased prevalence of symptoms consistent with SARS-CoV-2 infection, such as cough, increased sputum production or altered sense of smell or taste ${ }^{42}$. Evidence from a small number of studies indicates that current smokers may be more likely to present for testing, hence increasing the denominator in comparisons with never smokers and potentially inflating the rate of negative tests in current smokers. Infection positivity rates estimated among random samples are more informative. We identified one population study conducted in Hungary reporting on seroprevalence and smoking status ${ }^{43}$; however, the response rate was only $58.8 \%$ and the current smoking rate was 10 percentage points below national prevalence estimates, which raises some doubt about representativeness of the final sample. Similarly, a second representative population survey with results from three regions in France ${ }^{44}$ reported a current smoking rate of more than 10 percentage points below national prevalence ( $12 \%$ vs. $25 \%$ daily smoking prevalence $)^{45}$.

\section{4) Testing positive for SARS-CoV-2}

1. Smokers with COVID-19 may be less likely to receive a SARS-CoV-2 test or present to hospital due to lack of access to healthcare and may be more likely to die in the community from sudden complications (i.e. self-selection bias) and thus 
not be recorded ${ }^{46}$.

2. Diagnostic criteria for SARS-CoV-2 infection and COVID-19 have changed during the course of the pandemic ${ }^{47}$. It was not possible to extract details on the specific RT-PCR or antibody-based techniques or platforms used across the included studies due to reporting gaps. Different platforms have varying sensitivity and specificity to detect SARS-CoV2 infection. In addition, testing for acute infection requires swabbing of the mucosal epithelium, which may be disrupted in current smokers, potentially altering the sensitivity of assays ${ }^{48}$.

\section{5) Hospitalisation with COVID-19}

1. Reasons for hospitalisation vary by country and time in the pandemic. For example, early cases may have been hospitalised for isolation and quarantine reasons and not due to medical necessity. It is plausible this may have skewed early data towards less severe cases. In addition, the observed association between former smoking and greater disease severity may be explained by collider bias ${ }^{49}$, where conditioning on a collider (e.g. testing or hospitalisation) by design or analysis may introduce a spurious association between current or former smoking (a potential cause of testing or hospitalisation) and SARS-CoV-2 infection/adverse outcomes from COVID-19 (potentially exacerbated by smoking $)^{50}$.

2. The majority of included studies relied on EHRs as the source of information on smoking status. Research shows large discrepancies between EHRs and actual behaviour ${ }^{51}$. Known failings of EHRs include implausible longitudinal changes, such as former smokers being recorded as never smokers at subsequent hospital visits ${ }^{51}$. Misreporting on the part of the patient (perhaps due to perceived stigma) has also been observed, with biochemical measures showing higher rates of smoking compared with self-report in hospitalised patients in the US ${ }^{52}$. It is hence possible that underreporting of current and former smoking status in hospitals occurred across the included studies.

3. The majority of included studies were conducted in hospital settings. It is plausible that a non-trivial proportion of patients were infected with SARS-CoV-2 while being an inpatient for a different medical reason. If so, this may have biased the hospitalised populations towards older and more frail groups, who are less likely to be smokers ${ }^{53}$.

4. Individuals with severe COVID-19 symptoms may have stopped smoking immediately before admission to hospital and may therefore not have been recorded as current smokers (i.e. reverse causality).

\section{6) COVID-19 disease severity and death}

1. Given lack of knowledge of the disease progression and long-term outcomes of COVID-19, it is unclear whether studies conducted thus far in the pandemic have monitored patients for a sufficient time period to report complete survival outcomes or whether they are subject to early censoring. Adding to this, COVID-19 related mortality has been differentially defined across countries and epidemic phases. For example, in some UK reporting, death within 28 days of a COVID-19 diagnosis is required for attributing the cause of death to the virus. However, according to the UK Office for National Statistics, COVID-19 deaths are recorded only if this was stated on the death certificate.

2. If there is a protective effect of nicotine on COVID-19 disease outcomes, abrupt nicotine withdrawal upon 
hospitalisation may lead to worse disease outcomes including death ${ }^{12}$.

3. During periods of heightened demand of limited healthcare resources, current and former smokers with extensive comorbidities may have reduced priority for intensive care admission, thus leading to higher in-hospital mortality.

COVID-19 outcomes are currently limited to in-hospital death or survival to discharge. This binary outcome does not capture potential long-term morbidity attributed to COVID-19, such as stroke, amputation or acute cardiac events, which may be moderated by smoking status.

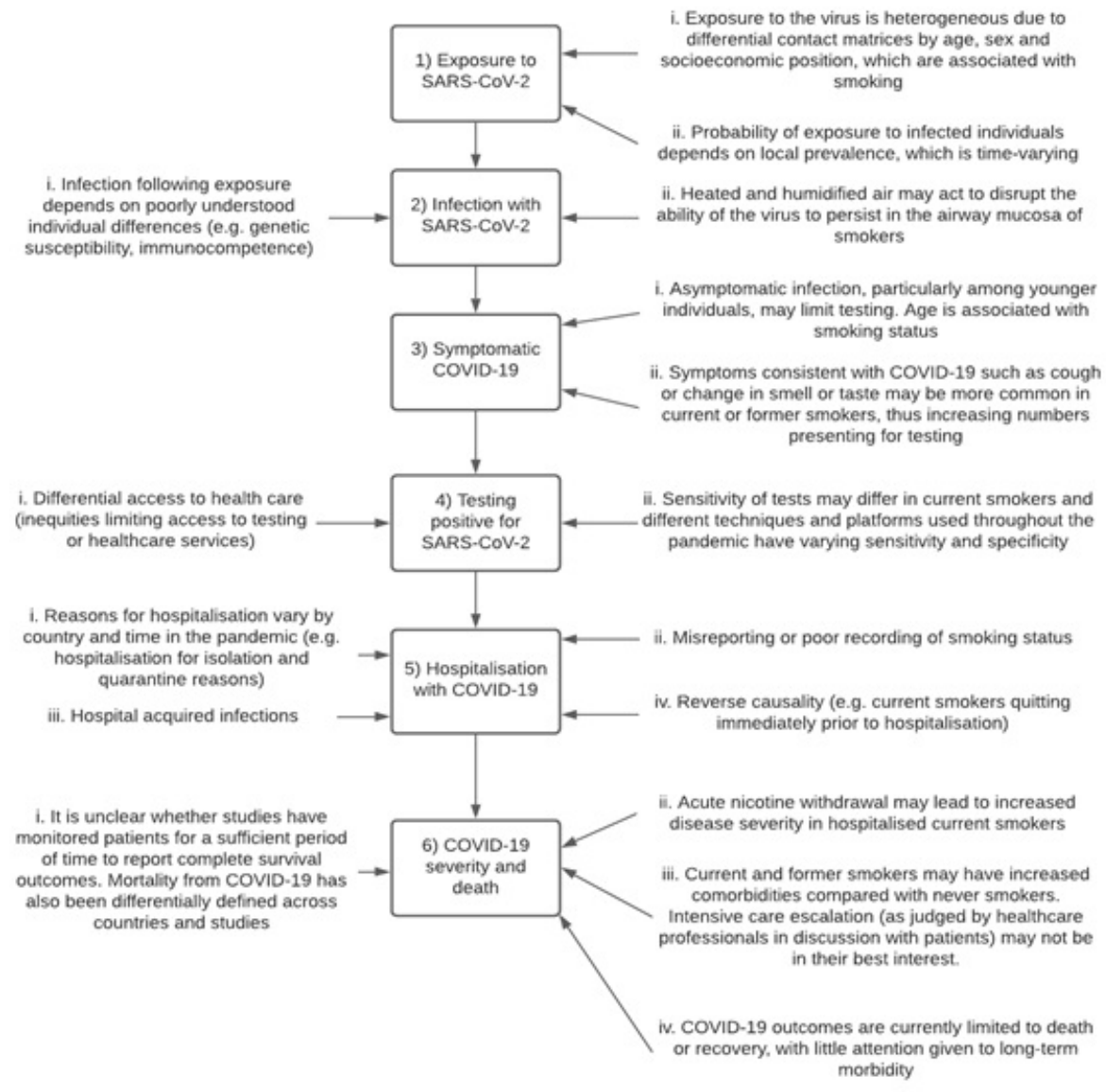

Figure 11. A schematic of some of the interpretation issues for the association of smoking status and COVID-19 infection, hospitalisation, disease severity and mortality. Numbers refer to the issues listed in the above section. Issues on the right-hand side relate explicitly to smoking status.

In addition, the emergence of new variants and strains of the SARS-CoV-2 virus may also change associations with risks factors, including smoking status.

\section{Limitations}


This living rapid evidence review was limited by having a single reviewer extracting data with a second independently verifying the data extracted to minimise errors, restricting the search to one electronic database and one pre-print server and by not including at least three large population surveys due to their reliance on self-reported suspected or confirmed SARS-CoV-2 infection (which means they do not meet our eligibility criteria) ${ }^{42,54,55}$. We also did not include a large, UKbased, representative seroprevalence study ${ }^{56}$ in our meta-analyses as the odds of testing positive in former smokers was not reported. However, the odds of infection for current smokers $(\mathrm{OR}=0.64,95 \% \mathrm{Cl}=0.58-0.71)$ was in concordance with the pooled estimate in our meta-analysis. Population surveys - particularly with linked data on confirmed infection or antibodies - will be included in future review versions to help mitigate some of the limitations of healthcare based observational studies. The comparisons of current and former smoking prevalence in the included studies with national prevalence estimates did not adjust observed prevalence for the demographic profile of those tested/admitted to hospital. Other reviews focused on this comparison have applied adjustments for sex and age, and continue to find lower than expected prevalence - notwithstanding the issues complicating interpretation described above ${ }^{17}$.

\section{Implications for research, policy and practice}

Further scientific research is needed to resolve the mixed findings summarised in our review. First, clinical trials of the posited therapeutic effect of nicotine could have important implications both for smokers and for improved understanding of how the SARS-CoV-2 virus causes disease in humans. Such trials should focus on medicinal nicotine (as smoked tobacco is a dirty delivery mechanism that could mask beneficial effects) and potentially differentiate between different modes of delivery (i.e. inhaled vs. ingested) since this can affect pharmacokinetics ${ }^{57}$ and potential therapeutic effects. A second research priority would be a large, representative (randomly sampled) population survey with a validated assessment of smoking status which distinguishes between recent and long-term ex-smokers - ideally biochemically verified - and assesses seroprevalence and links to health records.

In the meantime, public-facing messages about the possible protective effect of smoking or nicotine are premature. In our view, until there is further research, the quality of the evidence does not justify the huge risk associated with a message likely to reach millions of people that a lethal activity, such as smoking, may protect against COVID-19. It continues to be appropriate to recommend smoking cessation and emphasise the role of alternative nicotine products to support smokers to stop as part of public health efforts during COVID-19. At the very least, smoking cessation reduces acute risks from cardiovascular disease and could reduce demands on the healthcare system ${ }^{58}$. GPs and other healthcare providers can play a crucial role - brief, high-quality and free online training is available at National Centre for Smoking Cessation and Training.

\section{Conclusion}

Across 345 studies, recorded current but not past smoking prevalence was generally lower than national prevalence 
estimates. Current smokers were at reduced risk of testing positive for SARS-CoV-2 and former smokers were at increased risk of hospitalisation, disease severity and mortality compared with never smokers.

\section{Acknowledgements}

An original short review for the Royal College of Physicians was converted to an extended living review after a request by Martin Dockrell, Tobacco Control Lead, Public Health England. All scientific decisions were made by the authors independently of funders and external organisations. The authors would like to thank Rosemary Koper for her assistance in running the electronic searches and data extraction up until v7, and all authors who responded to requests for additional data.

\section{Declaration of conflicts of interest}

DS and OP have no conflicts of interest to declare. LS has received a research grant and honoraria for a talk and travel expenses from manufacturers of smoking cessation medications (Pfizer and Johnson \& Johnson). JB has received unrestricted research funding to study smoking cessation from companies who manufacture smoking cessation medications. All authors declare no financial links with tobacco companies or e-cigarette manufacturers or their representatives.

\section{Funding statement}

DS is supported by a PhD studentship from the UK Biotechnology and Biological Sciences Research Council [BB/M009513/1]. OP receives salary support from Cancer Research UK (C1417/A22962). JB, LS, \& OP are members of SPECTRUM, a UK Prevention Research Partnership Consortium (MR/S037519/1). UKPRP is an initiative funded by the UK Research and Innovation Councils, the Department of Health and Social Care (England) and the UK devolved administrations, and leading health research charities.

\section{Future review versions}

https://www.qeios.com/read/latest-UJR2AW

\section{Previous review versions}

Version 1: https://doi.org/10.32388/UJR2AW

Version 2: https://doi.org/10.32388/UJR2AW.3

Version 3: https://doi.org/10.32388/UJR2AW.4

Version 4: https://doi.org/10.32388/UJR2AW.5

Version 5: https://doi.org/10.32388/UJR2AW.6

Version 6: https://doi.org/10.32388/UJR2AW.7 
Version 7: https://doi.org/10.1111/add.15276

Version 8: https://doi.org/10.32388/UJR2AW.9

Version 9: https://doi.org/10.32388/UJR2AW.10

\section{Data availability}

All data contributing to the current and future review versions are available here: https://doi.org/10.6084/m9.figshare.12756020

All code required to reproduce the current and future analyses are available here:

https://doi.org/10.5281/zenodo.4002046

\section{References}

1 Guan W, Ni Z, Hu YY, et al. Clinical Characteristics of Coronavirus Disease 2019 in China. N Engl J Med 2020; : NEJMoa2002032.

2 Hoffmann M, Kleine-Weber H, Schroeder S, et al. SARS-CoV-2 Cell Entry Depends on ACE2 and TMPRSS2 and Is Blocked by a Clinically Proven Protease Inhibitor. Cell 2020; published online March 5. DOI:10.1016/j.cell.2020.02.052. 3 Brake SJ, Barnsley K, Lu W, McAlinden KD, Eapen MS, Sohal SS. Smoking Upregulates Angiotensin-Converting Enzyme-2 Receptor: A Potential Adhesion Site for Novel Coronavirus SARS-CoV-2 (Covid-19). J Clin Med 2020 Vol 9 Page 841 2020; 9: 841.

4 Cai G. Bulk and Single-Cell Transcriptomics Identify Tobacco-Use Disparity in Lung Gene Expression of ACE2, the Receptor of 2019-nCov. 2020; published online March 2. DOI:10.20944/PREPRINTS202002.0051.V3.

5 Oakes JM, Fuchs RM, Gardner JD, Lazartigues E, Yue X. Nicotine and the renin-angiotensin system. Am. J. Physiol. - Regul. Integr. Comp. Physiol. 2018; 315: R895-906.

6 Denholm JT, Gordon CL, Johnson PD, et al. Hospitalised adult patients with pandemic (H1N1) 2009 influenza in Melbourne, Australia. Med J Aust 2010; 192: 84-6.

7 Abadom TR, Smith AD, Tempia S, Madhi SA, Cohen C, Cohen AL. Risk factors associated with hospitalisation for influenza-associated severe acute respiratory illness in South Africa: A case-population study. Vaccine 2016; 34: 564955.

8 Almirall J, González CA, Balanzó X, Bolíbar I. Proportion of community-acquired pneumonia cases attributable to tobacco smoking. Chest 1999; 116: 375-9.

9 Feldman C, Anderson R. Cigarette smoking and mechanisms of susceptibility to infections of the respiratory tract and other organ systems. J. Infect. 2013; 67: 169-84.

10 Dye JA, Adler KB. Occasional review Effects of cigarette smoke on epithelial cells of the respiratory tract. Thorax 1994; 49: 825-34.

11 Vardavas Cl, Nikitara K. COVID-19 and smoking: A systematic review of the evidence. Tob Induc Dis 2020; 18: 20.

12 Farsalinos K, Niaura R, Le Houezec J, et al. Editorial: Nicotine and SARS-CoV-2: COVID-19 may be a disease of the nicotinic cholinergic system. Toxicol Rep 2020; published online April. DOI:10.1016/j.toxrep.2020.04.012.

13 Emami A, Javanmardi F, Pirbonyeh N, Akbari A. Prevalence of Underlying Diseases in Hospitalized Patients with 
COVID-19: a Systematic Review and Meta-Analysis. Arch Acad Emerg Med 2020; 8: e35.

14 Alqahtani JS, Oyelade T, Aldhahir AM, et al. Prevalence, Severity and Mortality associated with COPD and Smoking in patients with COVID-19: A Rapid Systematic Review and Meta-Analysis. medRxiv 2020; : 2020.03.25.20043745.

15 Patanavanich R, Glantz SA. Smoking is Associated with COVID-19 Progression: A Meta-Analysis. medRxiv 2020. DOI:10.14171/j.2095-5944.sg.2014.02.004.

16 Berlin I, Thomas D, Le Faou A-L, Cornuz J. COVID-19 and Smoking. Nicotine Tob Res DOI:10.1093/NTR/NTAA059.

17 Farsalinos K, Barbouni A, Niaura R. Systematic review of the prevalence of current smoking among hospitalized COVID-19 patients in China: could nicotine be a therapeutic option? Intern Emerg Med 2020; published online May 9. DOI:10.1007/s11739-020-02355-7.

18 Grundy* EJ, Suddek* T, Filippidis FT, Majeed A, Coronini-Cronberg S. Smoking, SARS-CoV-2 and COVID-19: A review of reviews considering implications for public health policy and practice. Tob Induc Dis 2020; 18. DOI:10.18332/tid/124788.

19 Elliott JH, Turner T, Clavisi O, et al. Living Systematic Reviews: An Emerging Opportunity to Narrow the EvidencePractice Gap. PLoS Med 2014; 11. DOI:10.1371/journal.pmed.1001603.

20 Tricco AC, Antony J, Zarin W, et al. A scoping review of rapid review methods. BMC Med 2015; 13: 224.

21 Simons D, Brown J, Shahab L, Perski O. Smoking and COVID-19: Rapid evidence review for the Royal College of Physicians, London (UK). Qeios 2020; published online April 1. DOI:10.32388/VGJCUN.

22 Simons D, Shahab L, Brown J, Perski O. The association of smoking status with SARS-CoV-2 infection, hospitalisation and mortality from COVID-19: A living rapid evidence review with Bayesian meta-analyses (version 7). Addiction; n/a. DOI:10.1111/add.15276.

23 R Core Team. The R Project for Statistical Computing. 2013; : 1-12.

24 Bürkner P-C. Advanced Bayesian Multilevel Modeling with the R Package brms. ArXiv170511123 Stat2017; published online Oct 15. http://arxiv.org/abs/1705.11123 (accessed July 26, 2020).

25 Simons D, Shahab L, Brown J, Perski O. The association of smoking status with SARS-CoV-2 infection, hospitalisation and mortality from COVID-19: A living rapid evidence review (version 5). Qeios 2020; published online July 1. DOI:10.32388/UJR2AW.6.

26 Miyara M, Tubach F, Martinez V, et al. Low rate of daily smokers in patients with symptomatic COVID-19. medrxiv 2020; : 2020.06.10.20127514.

27 Rimland CA, Morgan CE, Bell GJ, et al. Clinical characteristics and early outcomes in patients with COVID-19 treated with tocilizumab at a United States academic center. medRxiv 2020; : 2020.05.13.20100404.

28 Yannick Girardeau, Yoan Gallous, Guillaume de Bonnecaze, et al. Confirmed central olfactory system lesions on brain MRI in COVID-19 patients with anosmia: a case-series | medRxiv. https://doi.org/10.1101/2020.07.08.20148692 (accessed Aug 25, 2020).

29 Ebinger J, Botwin GJ, Albert CM, et al. SARS-CoV-2 Seroprevalence Across a Diverse Cohort of Healthcare Workers. medRxiv 2020; : 2020.07.31.20163055. 
Islam MZ, Riaz BK, Islam AS, et al. Risk factors associated with morbidity and mortality outcomes of COVID-19 patients on the 14th and 28th day of the disease course: a retrospective cohort study in Bangladesh. medRxiv 2020; : 2020.08.17.20176586.

31 Gallichotte EN, Quicke KM, Sexton NR, et al. Longitudinal Surveillance for SARS-CoV-2 Among Staff in Six Colorado Long-Term Care Facilities: Epidemiologic, Virologic and Sequence Analysis. medRxiv 2020; : 2020.06.08.20125989.

32 Rentsch CT, Kidwai-Khan F, Tate JP, et al. Covid-19 Testing, Hospital Admission, and Intensive Care Among 2,026,227 United States Veterans Aged 54-75 Years. medRxiv 2020; : 2020.04.09.20059964.

33 Odani S. Tobacco Product Use Among Military Veterans - United States, 2010-2015. MMWR Morb Mortal Wkly Rep 2018; 67. DOl:10.15585/mmwr.mm6701a2.

34 Niedzwiedz CL, O'Donnell CA, Jani BD, et al. Ethnic and socioeconomic differences in SARS-CoV-2 infection: prospective cohort study using UK Biobank. BMC Med 2020; 18: 160.

35 Trubiano JA, Vogrin S, Smibert OC, et al. COVID-MATCH65 - A prospectively derived clinical decision rule for severe acute respiratory syndrome coronavirus 2. medRxiv 2020; : 2020.06.30.20143818.

36 CMMID COVID-19 working group, Jarvis CI, Van Zandvoort K, et al. Quantifying the impact of physical distance measures on the transmission of COVID-19 in the UK. BMC Med 2020; 18: 124.

37 Fung HF, Martinez L, Alarid-Escudero F, et al. The household secondary attack rate of SARS-CoV-2: A rapid review. Clin Infect Dis DOI:10.1093/cid/ciaa1558.

38 Conti C, de Marco A, Mastromarino P, Tomao P, Santoro MG. Antiviral Effect of Hyperthermic Treatment in Rhinovirus Infection. Antimicrob Agents Chemother 1999; 43: 822-9.

39 Schoeman D, Fielding BC. Coronavirus envelope protein: current knowledge. Virol J 2019; 16: 69.

40 Buitrago-Garcia D, Egli-Gany D, Counotte MJ, et al. Occurrence and transmission potential of asymptomatic and presymptomatic SARS-CoV-2 infections: A living systematic review and meta-analysis. PLOS Med 2020; 17 : e1003346.

41 Kronbichler A, Kresse D, Yoon S, Lee KH, Effenberger M, Shin Jl. Asymptomatic patients as a source of COVID-19 infections: A systematic review and meta-analysis. Int J Infect Dis 2020; 98: 180-6.

42 Hopkinson NS, Rossi NN, Moustafa JE-SSE, et al. Current tobacco smoking and risk from COVID-19 results from a population symptom app in over 2.4 million people. medrxiv 2020; 44: 2020.05.18.20105288.

43 Merkely B, Szabó AJ, Kosztin A, et al. Novel coronavirus epidemic in the Hungarian population, a cross-sectional nationwide survey to support the exit policy in Hungary. GeroScience 2020; published online July 17.

DOI:10.1007/s11357-020-00226-9.

44 Carrat F, Lamballerie X de, Rahib D, et al. Seroprevalence of SARS-CoV-2 among adults in three regions of France following the lockdown and associated risk factors: a multicohort study. medRxiv 2020; : 2020.09.16.20195693.

45 Andler R. BAISSE DE LA PRÉVALENCE DU TABAGISME QUOTIDIEN PARMI LES ADULTES : RÉSULTATS DU BAROMÈTRE DE SANTÉ PUBLIQUE FRANCE 2018 / REDUCTION OF DAILY SMOKING RATE AMONG ADULTS: RESULTS FROM THE 2018 SANTÉ PUBLIQUE FRANCE HEALTH BAROMETER. $2019 ;$ : 7. 46 STS top line findings_Sep 20.pptx. Google Docs. https://docs.google.com/presentation/d/e/2PACX1vQmMeD0wPM7iEawo39m2QZnVBOo2e8YIQPjm2R0OgYBvKhBbYqnarSn5vxyk3-56w/embed? 
start=false\&loop=false\&delayms=3000\&usp=embed_facebook (accessed Nov 3, 2020).

47 World Health Organisation. Laboratory testing for 2019 novel coronavirus (2019-nCoV) in suspected human cases. https://www.who.int/publications-detail-redirect/10665-331501 (accessed July 29, 2020).

48 de Lusignan S, Dorward J, Correa A, et al. Risk factors for SARS-CoV-2 among patients in the Oxford Royal College of General Practitioners Research and Surveillance Centre primary care network: a cross-sectional study. Lancet Infect Dis 2020; 0. DOI:10.1016/S1473-3099(20)30371-6.

49 Griffith G, Morris TT, Tudball M, et al. Collider bias undermines our understanding of COVID-19 disease risk and severity. medRxiv 2020; : 2020.05.04.20090506.

50 Murray E. Causation in smoking and COVID-19. Twitter. 2020.

https://twitter.com/EpiEllie/status/1258607277357006849?s=20.

51 Polubriaginof F, Salmasian H, Albert DA, Vawdrey DK. Challenges with Collecting Smoking Status in Electronic Health Records. AMIA Annu Symp Proc AMIA Symp 2017; 2017: 1392-400.

52 Benowitz NL, Schultz KE, Haller CA, Wu AHB, Dains KM, Jacob P. Prevalence of smoking assessed biochemically in an urban public hospital: a rationale for routine cotinine screening. Am J Epidemiol 2009; 170: 885-91.

53 Mangera Z, Lewis A, Hutchinson J, Searle L, Agrawal S. Smoking prevalence in UK hospital admissions from a national observational study. Eur Respir J 2017; 50. DOI:10.1183/1393003.congress-2017.PA1268.

54 Bowyer RCE, Varsavsky T, Carole H. Geo-social gradients in predicted COVID-19 prevalence and severity in Great Britain : results from Affiliations: Corresponding authors : Understanding the geographical distribution of COVID-19 through the general population is key to the provision of ade. 2020.

55 Jackson SE, Brown J, Shahab L, Steptoe A, Fancourt D. COVID-19, smoking, and inequalities: a cross-sectional survey of adults in the UK. Submitted 2020.

56 Ward H, Atchison CJ, Whitaker M, et al. Antibody prevalence for SARS-CoV-2 in England following first peak of the pandemic: REACT2 study in 100,000 adults. medRxiv 2020; : 2020.08.12.20173690.

57 Shahab L, Brose LS, West R. Novel delivery systems for nicotine replacement therapy as an aid to smoking cessation and for harm reduction: Rationale, and evidence for advantages over existing systems. CNS Drugs 2013; 27: 1007-19.

58 Stead LF, Buitrago D, Preciado N, Sanchez G, Hartmann-Boyce J, Lancaster T. Physician advice for smoking cessation. Cochrane Database Syst. Rev. 2013; 2017. DOI:10.1002/14651858.CD000165.pub4.

59 Guan W, Liang W, Zhao Y, et al. Comorbidity and its impact on 1590 patients with COVID-19 in China: a nationwide analysis. Eur Respir J 2020; 55. DOI:10.1183/13993003.00547-2020.

60 Lian J, Jin X, Hao S, et al. Analysis of Epidemiological and Clinical Features in Older Patients With Coronavirus Disease 2019 (COVID-19) Outside Wuhan. Clin Infect Dis DOI:10.1093/cid/ciaa242.

61 Jin X, Lian J-S, Hu J-H, et al. Epidemiological, clinical and virological characteristics of 74 cases of coronavirusinfected disease 2019 (COVID-19) with gastrointestinal symptoms. Gut2020; 69: 1002-9.

62 Chen T, Wu D, Chen H, et al. Clinical characteristics of 113 deceased patients with coronavirus disease 2019: retrospective study. BMJ 2020; 368. DOI:10.1136/bmj.m1091.

63 Zhou F, Yu T, Du R, et al. Clinical course and risk factors for mortality of adult inpatients with COVID-19 in Wuhan, 
China: a retrospective cohort study. The Lancet 2020; 395: 1054-62.

64 Mo P, Xing Y, Xiao Y, et al. Clinical characteristics of refractory COVID-19 pneumonia in Wuhan, China. Clin Infect Dis DOI:10.1093/cid/ciaa270.

65 Zhang J, Dong X, Cao Y, et al. Clinical characteristics of 140 patients infected with SARS-CoV-2 in Wuhan, China. Allergy 2020; 75: 1730-41.

66 Wan S, Xiang Y, Fang W, et al. Clinical features and treatment of COVID-19 patients in northeast Chongqing. $J$ Med Virol 2020; 92: 797-806.

67 Liu W, Tao Z-W, Wang L, et al. Analysis of factors associated with disease outcomes in hospitalized patients with 2019 novel coronavirus disease. Chin Med J (Engl) 2020; 133: 1032-8.

68 Huang C, Wang Y, Li X, et al. Clinical features of patients infected with 2019 novel coronavirus in Wuhan, China. The Lancet 2020; 395: 497-506.

69 Zhang X, Cai H, Hu J, et al. Epidemiological, clinical characteristics of cases of SARS-CoV-2 infection with abnormal imaging findings. Int $J$ Infect Dis 2020; 94: 81-7.

70 Guo T, Fan Y, Chen M, et al. Cardiovascular Implications of Fatal Outcomes of Patients With Coronavirus Disease 2019 (COVID-19). JAMA Cardiol 2020; 5: 811-8.

71 Liu R, Ming X, Xu O, et al. Association of Cardiovascular Manifestations with In-hospital Outcomes in Patients with COVID-19: A Hospital Staff Data. medRxiv 2020; : 2020.02.29.20029348.

72 Huang Y, Yang R, Xu Y, Gong P. Clinical characteristics of 36 non-survivors with COVID-19 in Wuhan, China. medRxiv 2020; : 2020.02.27.20029009.

$73 \mathrm{Xu} \mathrm{H}, \mathrm{Hou} \mathrm{K}, \mathrm{Xu} \mathrm{H}$, et al. Acute Myocardial Injury of Patients with Coronavirus Disease 2019. medRxiv 2020; : 2020.03.05.20031591.

74 Li J, Li S, Cai Y, et al. Epidemiological and Clinical Characteristics of 17 Hospitalized Patients with 2019 Novel Coronavirus Infections Outside Wuhan, China. medRxiv 2020; : 2020.02.11.20022053.

75 Hu L, Chen S, Fu Y, et al. Risk Factors Associated with Clinical Outcomes in 323 COVID-19 Patients in Wuhan, China. medRxiv 2020; : 2020.03.25.20037721.

76 Wang R, Pan M, Zhang X, et al. Epidemiological and clinical features of 125 Hospitalized Patients with COVID-19 in Fuyang, Anhui, China. Int J Infect Dis 2020; 95: 421-8.

77 CDCMMWR. Preliminary Estimates of the Prevalence of Selected Underlying Health Conditions Among Patients with Coronavirus Disease 2019 - United States, February 12-March 28, 2020. MMWR Morb Mortal Wkly Rep 2020; 69. DOI:10.15585/mmwr.mm6913e2.

78 Dong X, Cao Y, Lu X, et al. Eleven faces of coronavirus disease 2019. Allergy 2020; 75: 1699-709.

79 Kim ES, Chin BS, Kang CK, et al. Clinical Course and Outcomes of Patients with Severe Acute Respiratory Syndrome Coronavirus 2 Infection: a Preliminary Report of the First 28 Patients from the Korean Cohort Study on COVID19. J Korean Med Sci 2020; 35. DOI:10.3346/jkms.2020.35.e142.

80 Shi Y, Yu X, Zhao H, Wang H, Zhao R, Sheng J. Host susceptibility to severe COVID-19 and establishment of a host risk score: findings of 487 cases outside Wuhan. Crit Care 2020; 24: 108.

81 Yang X, Yu Y, Xu J, et al. Clinical course and outcomes of critically ill patients with SARS-CoV-2 pneumonia in 
Wuhan, China: a single-centered, retrospective, observational study. Lancet Respir Med 2020; 8: 475-81.

82 Argenziano MG, Bruce SL, Slater CL, et al. Characterization and clinical course of 1000 patients with coronavirus disease 2019 in New York: retrospective case series. BMJ 2020; 369. DOl:10.1136/bmj.m1996.

83 Solís P, Carreňo H. COVID-19 Fatality and Comorbidity Risk Factors among Confirmed Patients in Mexico. Epidemiology, 2020 DOI:10.1101/2020.04.21.20074591.

84 Richardson S, Hirsch JS, Narasimhan M, et al. Presenting Characteristics, Comorbidities, and Outcomes Among 5700 Patients Hospitalized With COVID-19 in the New York City Area. JAMA 2020; 323: 2052-9.

85 Fontanet A, Tondeur L, Madec Y, et al. Cluster of COVID-19 in northern France: A retrospective closed cohort study. medRxiv 2020; : 2020.04.18.20071134.

86 Zheng KI, Gao F, Wang X-B, et al.Letter to the Editor: Obesity as a risk factor for greater severity of COVID-19 in patients with metabolic associated fatty liver disease. Metabolism 2020; 108: 154244.

87 Liao Y, Feng Y, Wang B, et al. Clinical Characteristics and Risk factors for developed COVID-19 patients transferring to designated hospital from Jianghan Fangcang shelter Hospital: a retrospective, observational study. medRxiv 2020; : 2020.04.21.20074724.

88 Gil-Agudo A, Rodriguez-Cola M, Jimenez-Velasco I, et al. Clinical features of coronavirus disease 2019 (COVID19) in a cohort of patients with disability due to spinal cord injury. medRxiv 2020; : 2020.04.20.20072918.

89 Shi P, Ren G, Yang J, et al. Clinical characteristics of imported and second-generation COVID-19 cases outside Wuhan, China: A multicenter retrospective study. medRxiv 2020; : 2020.04.19.20071472.

90 Hadjadj J, Yatim N, Barnabei L, et al. Impaired type I interferon activity and exacerbated inflammatory responses in severe Covid-19 patients. medRxiv 2020; : 2020.04.19.20068015.

91 Gold JAW, Wong KK, Szablewski CM, et al. Characteristics and Clinical Outcomes of Adult Patients Hospitalized with COVID-19 - Georgia, March 2020. MMWR Morb Mortal Wkly Rep 2020; 69: 545-50.

92 Yu T, Cai S, Zheng Z, et al. Association Between Clinical Manifestations and Prognosis in Patients with COVID-19. Clin Ther 2020; 42: 964-72.

93 Zheng Y, Xiong C, Liu Y, et al. Epidemiological and clinical characteristics analysis of COVID-19 in the surrounding areas of Wuhan, Hubei Province in 2020. Pharmacol Res 2020; 157: 104821.

94 Rica R de la, Borges M, Aranda M, et al. Low albumin levels are associated with poorer outcomes in a case series of COVID-19 patients in Spain: a retrospective cohort study. medRxiv 2020; : 2020.05.07.20094987.

95 Yin R, Yang Z, Wei Y, et al. Clinical characteristics of 106 patients with neurological diseases and co-morbid coronavirus disease 2019: a retrospective study. medRxiv 2020; : 2020.04.29.20085415.

96 Shi H, Zuo Y, Yalavarthi S, et al. Neutrophil calprotectin identifies severe pulmonary disease in COVID-19. medRxiv 2020; : 2020.05.06.20093070.

97 Cho ER, Slutsky AS, Jha P. Smoking and the risk of COVID-19 infection in the UK Biobank Prospective Study. medRxiv 2020; : 2020.05.05.20092445.

98 Allenbach $\mathrm{Y}$, Saadoun D, Maalouf $\mathrm{G}$, et al. Multivariable prediction model of intensive care unit transfer and death: a French prospective cohort study of COVID-19 patients. medRxiv 2020; : 2020.05.04.20090118.

99 Robilotti EV, Babady NE, Mead PA, et al. Determinants of Severity in Cancer Patients with COVID-19 IIIness. 
medRxiv 2020; : 2020.05.04.20086322.

100 Williamson EJ, Walker AJ, Bhaskaran K, et al. OpenSAFELY: factors associated with COVID-19 death in 17 million patients. Nature 2020; : 1-11.

101 Borobia AM, Carcas AJ, Arnalich F, et al. A Cohort of Patients with COVID-19 in a Major Teaching Hospital in Europe. J Clin Med 2020; 9: 1733.

102 Giacomelli A, Ridolfo AL, Milazzo L, et al. 30-day mortality in patients hospitalized with COVID-19 during the first wave of the Italian epidemic: A prospective cohort study. Pharmacol Res 2020; 158: 104931.

103 Shah SJ, Barish PN, Prasad PA, et al. Clinical features, diagnostics, and outcomes of patients presenting with acute respiratory illness: a comparison of patients with and without COVID-19. medRxiv 2020; : 2020.05.02.20082461. 104 Kolin DA, Kulm S, Elemento O. Clinical and Genetic Characteristics of Covid-19 Patients from UK Biobank. medRxiv 2020; : 2020.05.05.20075507.

105 Lubetzky M, Aull M, Craig-Shapiro R, et al. Kidney Allograft Recipients Diagnosed with Coronavirus Disease-2019: A Single Center Report. medRxiv 2020; : 2020.04.30.20086462.

106 Goyal P, Choi JJ, Pinheiro LC, et al. Clinical Characteristics of Covid-19 in New York City. N Engl J Med 2020; 382: 2372-4.

107 Feng Y, Ling Y, Bai T, et al. COVID-19 with Different Severities: A Multicenter Study of Clinical Features. Am J Respir Crit Care Med 2020; 201: 1380-8.

108 Yao Q, Wang P, Wang X, Qie G, Chu Y. A retrospective study of risk factors for severe acute respiratory syndrome coronavirus 2 infections in hospitalized adult patients. DOI:10.20452/pamw.15312.

109 Sami R, Soltaninejad F, Amra B, et al. A one-year hospital-based prospective COVID-19 open-cohort in the Eastern Mediterranean region: The Khorshid COVID Cohort (KCC) study. medRxiv 2020; : 2020.05.11.20096727. 110 Almazeedi S, Youha SA, Jamal MH, et al. Clinical Characteristics, Risk Factors and Outcomes Among the First Consecutive 1,096 Patients Diagnosed with COVID-19: The Kuwait Experience. medRxiv 2020; : 2020.05.09.20096495.

111 Carrillo-Vega MF, Salinas-Escudero G, Garcia-Peña C, Gutierrez-Robledo LM, Parra-Rodriguez L. Early estimation of the risk factors for hospitalisation and mortality by COVID-19 in Mexico. medRxiv 2020; : 2020.05.11.20098145.

112 Yanover C, Mizrahi B, Kalkstein N, et al. What factors increase the risk of complications in SARS-CoV-2 positive patients? A cohort study in a nationwide Israeli health organization. medRxiv 2020; : 2020.05.07.20091652.

113 Hamer M, Kivimäki M, Gale CR, Batty GD. Lifestyle risk factors, inflammatory mechanisms, and COVID-19 hospitalization: A community-based cohort study of 387,109 adults in UK. Brain Behav Immun 2020; 87: 184-7.

114 Regina J, Papadimitriou-Olivgeris M, Burger R, et al. Epidemiology, risk factors and clinical course of SARS-CoV-2 infected patients in a Swiss university hospital: an observational retrospective study. medRxiv 2020; : 2020.05.11.20097741.

115 de Lusignan S, Dorward J, Correa A, et al. Risk factors for SARS-CoV-2 among patients in the Oxford Royal College of General Practitioners Research and Surveillance Centre primary care network: a cross-sectional study. Lancet Infect Dis 2020; : S1473309920303716.

116 Targher G, Mantovani A, Wang X-B, et al. Patients with diabetes are at higher risk for severe illness from COVID19. Diabetes Metab 2020; published online May 13. DOI:10.1016/j.diabet.2020.05.001. 
117 Valenti L, Bergna A, Pelusi S, et al. SARS-CoV-2 seroprevalence trends in healthy blood donors during the COVID-19 Milan outbreak. medRxiv 2020; : 2020.05.11.20098442.

118 Feuth T, Saaresranta T, Karlsson A, et al. Is sleep apnoea a risk factor for Covid-19? Findings from a retrospective cohort study. medRxiv 2020; : 2020.05.14.20098319.

119 Ge H, Zhu M, Du J, et al. Cardiac Structural and Functional Characteristics in Patients with Coronavirus Disease 2019: A Serial Echocardiographic Study. medRxiv 2020; : 2020.05.12.20095885.

120 Parrotta E, Kister I, Charvet L, et al.COVID-19 outcomes in MS: Observational study of early experience from NYU Multiple Sclerosis Comprehensive Care Center. Neurol - Neuroimmunol Neuroinflammation 2020; 7: e835.

121 Shekhar R, Sheikh AB, Upadhyay S, Atencio J, Kapuria D. Early experience with COVID-19 patients at academic hospital in Southwestern United States. Infect Dis 2020; 52: 596-9.

122 Mejia-Vilet JM, Cordova-Sanchez BM, Fernandez-Camargo D, Mendez-Perez RA, Morales-Buenrostro LE, Hernandez-Gilsoul T. A Risk Score to Predict Admission to Intensive Care Unit in Patients With COVID-19: The ABCGOALS Score. medRxiv 2020; : 2020.05.12.20099416.

123 Chen C, Jiang J, Xu X, Hu Y, Hu Y, Zhao Y. Dynamic liver function indexes monitoring and clinical characteristics in three types of COVID-19 patients. medRxiv 2020; : 2020.05.13.20099614.

124 Li J, Chen Y, Chen S, et al. Derivation and validation of a prognostic model for predicting in-hospital mortality in patients admitted with COVID-19 in Wuhan, China: the PLANS (Platelet Lymphocyte Age Neutrophil Sex) model. medRxiv $2020 ;:$ :2020.05.13.20100370.

125 Palaiodimos L, Kokkinidis DG, Li W, et al. Severe obesity, increasing age and male sex are independently associated with worse in-hospital outcomes, and higher in-hospital mortality, in a cohort of patients with COVID-19 in the Bronx, New York. Metabolism 2020; 108: 154262.

126 Ip A, Berry DA, Hansen E, et al. Hydroxychloroquine and Tocilizumab Therapy in COVID-19 Patients - An Observational Study. medRxiv 2020; : 2020.05.21.20109207.

127 Heili-Frades S. COVID-19 Outcomes in 4712 consecutively confirmed SARS-CoV2 cases in the city of Madrid. medRxiv https://doi.org/10.1101/2020.05.22.20109850 (accessed July 27, 2020).

128 Vaquero LM, Barrado MES, Escobar D, et al. C-Reactive protein and SOFA score as early predictors of critical care requirement in patients with COVID-19 pneumonia in Spain. medRxiv 2020; : 2020.05.22.20110429.

129 Kim L, Garg S, O’Halloran A, et al. Interim Analysis of Risk Factors for Severe Outcomes among a Cohort of Hospitalized Adults Identified through the U.S. Coronavirus Disease 2019 (COVID-19)-Associated Hospitalization Surveillance Network (COVID-NET). medRxiv 2020; : 2020.05.18.20103390.

130 Wu MA, Fossali T, Pandolfi L, et al.COVID-19: the key role of pulmonary capillary leakage. An observational cohort study. medRxiv 2020; : 2020.05.17.20104877.

131 Shi Q, Zhao K, Yu J, et al. Clinical characteristics of 101 COVID-19 nonsurvivors in Wuhan, China: a retrospective study. medRxiv 2020; : 2020.03.04.20031039.

132 Al-Hindawi A, Sokhi J, Cuddihy J, et al. COVID-19 in London, a Case Series Demonstrating Late Improvement in Survivors. medRxiv 2020; : 2020.05.16.20103853.

133 Basse C, Diakite S, Servois V, et al. Characteristics and outcome of SARS-CoV-2 infection in cancer patients. 
medRxiv 2020; : 2020.05.14.20101576.

134 Freites D, Leon L, Mucientes A, et al. Risk factors for hospital admission related to COVID-19 in inflammatory rheumatic diseases. medRxiv 2020; : 2020.05.14.20101584.

135 Alshami AA, Alattas RA, Anan HF, et al.Silent Disease and Loss of Taste and Smell are Common Manifestations of SARS-COV-2 Infection in a Quarantine Facility: First report from Saudi Arabia. medRxiv 2020; : 2020.05.13.20100222.

136 Berumen J, Schmulson M, Alegre J, et al. Risk of infection and hospitalization by Covid-19 in Mexico: a casecontrol study. medRxiv 2020; : 2020.05.24.20104414.

137 Gianfrancesco M, Hyrich KL, Al-Adely S, et al. Characteristics associated with hospitalisation for COVID-19 in people with rheumatic disease: data from the COVID-19 Global Rheumatology Alliance physician-reported registry. Ann Rheum Dis 2020; 79: 859-66.

138 Li J, Long X, Zhu C, et al. Olfactory Dysfunction in Recovered Coronavirus Disease 2019 (COVID-19) Patients. Mov Disord; n/a. DOI:10.1002/mds.28172.

139 Batty GD, Deary I, Luciano M, Altschul D, Kivimaki M, Gale C. Psychosocial factors and hospitalisations for COVID19: Prospective cohort study of the general population. medRxiv 2020; : 2020.05.29.20100735.

140 Israel A, Feldhamer I, Lahad A, Levin-Zamir D, Lavie G. Smoking and the risk of COVID-19 in a large observational population study. medRxiv 2020; : 2020.06.01.20118877.

141 Valle DMD, Kim-schulze S, Hsin-hui H, et al. An inflammatory cytokine signature helps predict COVID-19 severity and death. medRxiv 2020; : 2020.05.28.20115758.

142 Chaudhry F, Bulka H, Rathnam AS, et al. COVID-19 in Multiple Sclerosis Patients and Risk Factors for Severe Infection. medRxiv 2020; : 2020.05.27.20114827.

143 Louis S, Dhawan A, Newey C, et al. Continuous Electroencephalography (cEEG) Characteristics and Acute Symptomatic Seizures in COVID-19 Patients. medRxiv 2020; : 2020.05.26.20114033.

144 Soto-Mota A, Garza BAM, Rodriguez EM, et al. THE LOW-HARM SCORE FOR PREDICTING MORTALITY IN PATIENTS DIAGNOSED WITH COVID-19: A MULTICENTRIC VALIDATION STUDY. medRxiv 2020; : 2020.05.26.20111120.

145 Garibaldi BT, Fiksel J, Muschelli J, et al. Patient trajectories and risk factors for severe outcomes among persons hospitalized for COVID-19 in the Maryland/DC region. medRxiv 2020; : 2020.05.24.20111864.

146 Docherty AB, Harrison EM, Green CA, et al. Features of 20133 UK patients in hospital with covid-19 using the ISARIC WHO Clinical Characterisation Protocol: prospective observational cohort study. BMJ 2020; 369.

DOl:10.1136/bmj.m1985.

147 Boulware DR, Pullen MF, Bangdiwala AS, et al. A Randomized Trial of Hydroxychloroquine as Postexposure Prophylaxis for Covid-19. N Engl J Med 2020; published online June 3. DOI:10.1056/NEJMoa2016638.

148 Kuderer NM, Choueiri TK, Shah DP, et al. Clinical impact of COVID-19 on patients with cancer (CCC19): a cohort study. The Lancet2020; 395: 1907-18.

149 Romão VC, Oliveira-Ramos F, Cruz-Machado AR, et al. A COVID-19 outbreak in a rheumatology department upon the early days of the pandemic. medRxiv 2020; : 2020.06.05.20107011.

150 Giannouchos T, Sussman R, Mier JM, Poulas K, Farsalinos K. Characteristics and risk factors for COVID-19 
diagnosis and adverse outcomes in Mexico: an analysis of 89,756 laboratory-confirmed COVID-19 cases. medRxiv 2020; : 2020.06.04.20122481.

151 Ramlall V, Thangaraj P, Meydan C, et al. Identification of Immune complement function as a determinant of adverse SARS-CoV-2 infection outcome. medRxiv 2020; : 2020.05.05.20092452.

152 Wang B, Oekelen OV, Mouhieddine T, et al. A tertiary center experience of multiple myeloma patients with COVID19: lessons learned and the path forward. medRxiv 2020; : 2020.06.04.20122846.

153 Perrone F, Piccirillo MC, Ascierto PA, et al. Tocilizumab for patients with COVID-19 pneumonia. The TOCIVID-19 prospective phase 2 trial. medRxiv 2020; : 2020.06.01.20119149.

154 Sharma AK, Ahmed A, Baig VN, et al. Characteristics and Outcomes of Hospitalized Young Adults with Mild to Moderate Covid-19 at a University Hospital in India. medRxiv 2020; : 2020.06.02.20106310.

155 Eugen-Olsen J, Altintas I, Tingleff J, et al. Low levels of the prognostic biomarker suPAR are predictive of mild outcome in patients with symptoms of COVID-19 - a prospective cohort study. medRxiv 2020; : 2020.05.27.20114678.

156 Martinez-Portilla RJ, Sotiriadis A, Torres-Torres J, et al. Risk factors for mortality in pregnant women with SARSCoV-2 infection. medRxiv 2020; : 2020.05.31.20107276.

157 Raisi-Estabragh Z, McCracken C, Bethell MS, et al. Greater risk of severe COVID-19 in Black, Asian and Minority Ethnic populations is not explained by cardiometabolic, socioeconomic or behavioural factors, or by $25(\mathrm{OH})$-vitamin D status: study of 1326 cases from the UK Biobank. J Public Health DOI:10.1093/pubmed/fdaa095.

158 Luo H, Liu S, Wang Y, et al. Age differences in clinical features and outcomes in patients with COVID-19, Jiangsu, China: a retrospective, multi-center cohort study. medRxiv 2020; : 2020.06.01.20086025.

159 Houlihan CF, Vora N, Byrne T, et al.Pandemic peak SARS-CoV-2 infection and seroconversion rates in London frontline health-care workers. The Lancet2020; : S0140673620314847.

160 Cen Y, Chen X, Shen Y, et al. Risk factors for disease progression in patients with mild to moderate coronavirus disease 2019—a multi-centre observational study. Clin Microbiol Infect2020; : S1198743X20303414.

161 Klang E, Kassim G, Soffer S, Freeman R, Levin MA, Reich DL. Morbid Obesity as an Independent Risk Factor for COVID-19 Mortality in Hospitalized Patients Younger than 50. Obesity; n/a. DOI:10.1002/oby.22913.

162 Maraschini A, Corsi E, Salvatore MA, Donati S. Coronavirus and birth in Italy: results of a national population-based cohort study. medRxiv 2020; : 2020.06.11.20128652.

163 Wang A-L, Zhong X, Hurd Y. Comorbidity and Sociodemographic determinants in COVID-19 Mortality in an US Urban Healthcare System. medRxiv 2020; : 2020.06.11.20128926.

164 McQueenie R, Foster H, Jani BD, et al.Multimorbidity, Polypharmacy, and COVID-19 infection within the UK Biobank cohort. medRxiv 2020; : 2020.06.10.20127563.

165 Apea VJ, Wan YI, Dhairyawan R, et al. Ethnicity and outcomes in patients hospitalised with COVID-19 infection in East London: an observational cohort study. medRxiv 2020; : 2020.06.10.20127621.

166 Woolford SJ, D'angelo S, Curtis EM, et al. COVID-19 and associations with frailty and multimorbidity: a prospective analysis of UK Biobank participants. medRxiv 2020; : 2020.06.09.20126292.

167 Hultcrantz M, Richter J, Rosenbaum C, et al. COVID-19 infections and outcomes in patients with multiple myeloma in New York City: a cohort study from five academic centers. medRxiv 2020; : 2020.06.09.20126516. 
168 Rajter JC, Sherman M, Fatteh N, Vogel F, Sacks J, Rajter J-J. ICON (Ivermectin in COvid Nineteen) study: Use of Ivermectin is Associated with Lower Mortality in Hospitalized Patients with COVID19. medRxiv 2020; : 2020.06.06.20124461.

169 Lan F-Y, Suharlim C, Kales SN, Yang J. Association between SARS-CoV-2 infection, exposure risk and mental health among a cohort of essential retail workers in the United States. medRxiv 2020; : 2020.06.08.20125120.

170 Zeng H, Zhang T, He X, et al. Impact of Chronic Comorbidities on Progression and Prognosis in Patients with COVID-19: A Retrospective Cohort Study in 1031 Hospitalized Cases in Wuhan, China. medRxiv 2020; : 2020.06.14.20125997.

171 Suleyman G, Fadel RA, Malette KM, et al. Clinical Characteristics and Morbidity Associated With Coronavirus Disease 2019 in a Series of Patients in Metropolitan Detroit. JAMA Netw Open 2020; 3: e2012270-e2012270.

172 Chen L, Yu J, He W, et al. Risk factors for death in 1859 subjects with COVID-19. Leukemia 2020; : 1-11.

173 Garassino MC, Whisenant JG, Huang L-C, et al. COVID-19 in patients with thoracic malignancies (TERAVOLT): first results of an international, registry-based, cohort study. Lancet Oncol 2020; 21: 914-22.

174 Hernández-Garduño E. Obesity is the comorbidity more strongly associated for Covid-19 in Mexico. A case-control study. Obes Res Clin Pract2020; published online June 12. DOI:10.1016/j.orcp.2020.06.001.

175 Govind R, Freitas DF de, Pritchard MR, Hayes RD, MacCabe JH. Clozapine treatment and risk of COVID-19. medRxiv 2020; : 2020.06.17.20133595.

176 Sisó-Almirall A, Kostov B, Mas-Heredia M, et al. PROGNOSTIC FACTORS IN SPANISH COVID-19 PATIENTS: A CASE SERIES FROM BARCELONA. medRxiv 2020; : 2020.06.18.20134510.

177 Gu T, Mack JA, Salvatore M, et al. COVID-19 outcomes, risk factors and associations by race: a comprehensive analysis using electronic health records data in Michigan Medicine. medRxiv 2020; : 2020.06.16.20133140.

178 Kibler M, Carmona A, Marchandot B, et al. Risk and severity of COVID-19 and ABO blood group in transcatheter aortic valve patients. medRxiv 2020; : 2020.06.13.20130211.

179 Ikitimur H, Uysal BB, Cengiz M, et al. "Determining Host Factors Contributing to Disease Severity in a Family Cluster of 29 Hospitalized SARS-CoV-2 Patients: Could Genetic Factors Be Relevant in the Clinical Course of COVID19?". J Med Virol; n/a. DOI:10.1002/jmv.26106.

180 Sierpiński R, Pinkas J, Jankowski M, et al. Sex differences in the frequency of gastrointestinal symptoms and olfactory or taste disorders among 1,942 non-hospitalized patients with COVID-19. Pol Arch Intern Med 2020; published online June 3. DOI:10.20452/pamw.15414.

181 Zhou Y, He X, Zhang J, et al. Prolonged SARS-CoV-2 Viral Shedding in Patients with COVID-19 was Associated with Delayed Initiation of Arbidol Treatment: a retrospective cohort study. medRxiv 2020; : 2020.06.09.20076646.

182 Crovetto F, Crispi F, Llurba E, Figueras F, Gomez-Roig MD, Gratacos E. SEROPREVALENCE AND CLINICAL SPECTRUM OF SARS-CoV-2 INFECTION IN THE FIRST VERSUS THIRD TRIMESTER OF PREGNANCY. medRxiv $2020 ;:$ 2020.06.17.20134098.

183 Veras FP, Pontelli M, Silva C, et al.SARS-CoV-2 triggered neutrophil extracellular traps (NETs) mediate COVID-19 pathology. medRxiv 2020; : 2020.06.08.20125823.

184 Sterlin D, Mathian A, Miyara M, et al. IgA dominates the early neutralizing antibody response to SARS-CoV-2. 
medRxiv 2020; : 2020.06.10.20126532.

185 Rossi B, Nguyen LS, Zimmermann P, et al. Effect of tocilizumab in hospitalized patients with severe pneumonia COVID-19: a cohort study. medRxiv 2020; : 2020.06.06.20122341.

186 Duan L, Zhang S, Guo M, et al. Epidemiological and clinical characteristics in patients with SARS-CoV-2 antibody negative probable COVID-19 in Wuhan. medRxiv 2020; : 2020.06.18.20134619.

187 Martin-Jimenez P, Munoz-Garcia MI, Seoane D, et al. Cognitive impairment is a common comorbidity in COVID-19 deceased patients. A hospital-based retrospective cohort study. medRxiv 2020; : 2020.06.08.20125872.

188 Elezkurtaj S, Greuel S, Ihlow J, et al. Causes of Death and Comorbidities in Patients with COVID-19. medRxiv $2020 ;:$ 2020.06.15.20131540.

189 Lenka J, Chhabria MS, Sharma N, et al.Clinical characteristics and outcomes of critically ill patients with COVID-19 in a tertiary community hospital in upstate New York. medRxiv 2020; : 2020.06.18.20135046.

190 Olivares F, Munoz D, Fica A, et al.Covid-19 in Chile. The experience of a Regional reference Center. Preliminary report. medRxiv 2020; : 2020.06.14.20130898.

191 Salton F, Confalonieri P, Santus P, et al. Prolonged low-dose methylprednisolone in patients with severe COVID19 pneumonia. medRxiv 2020; : 2020.06.17.20134031.

192 Wei W, Ortwine JK, Mang NS, Joseph C, Hall BC, Prokesch BC. Limited Role for Antibiotics in COVID-19: Scarce Evidence of Bacterial Coinfection. medRxiv 2020; : 2020.06.16.20133181.

193 Zuo Y, Estes SK, Gandhi AA, et al. Prothrombotic antiphospholipid antibodies in COVID-19. medRxiv 2020; : 2020.06.15.20131607.

194 Killerby ME. Characteristics Associated with Hospitalization Among Patients with COVID-19 — Metropolitan Atlanta, Georgia, March-April 2020. MMWR Morb Mortal Wkly Rep 2020; 69. DOI:10.15585/mmwr.mm6925e1.

195 Petrilli CM, Jones SA, Yang J, et al. Factors associated with hospital admission and critical illness among 5279 people with coronavirus disease 2019 in New York City: prospective cohort study. BMJ 2020; 369.

DOI:10.1136/bmj.m1966.

196 Magagnoli J, Narendran S, Pereira F, et al. Outcomes of Hydroxychloroquine Usage in United States Veterans Hospitalized with COVID-19. Med 2020; : S2666634020300064.

197 Bello-Chavolla OY, Bahena-López JP, Antonio-Villa NE, et al. Predicting Mortality Due to SARS-CoV-2: A Mechanistic Score Relating Obesity and Diabetes to COVID-19 Outcomes in Mexico. J Clin Endocrinol Metab 2020; 105. DOI:10.1210/clinem/dgaa346.

198 Zuo Y, Zuo M, Yalavarthi S, et al. Neutrophil extracellular traps and thrombosis in COVID-19. medRxiv 2020; : 2020.04.30.20086736.

199 Sigel K, Swartz T, Golden E, et al.Covid-19 and People with HIV Infection: Outcomes for Hospitalized Patients in New York City. Clin Infect Dis DOI:10.1093/cid/ciaa880.

200 Nguyen AB, Upadhyay GA, Chung B, et al. Outcomes and Cardiovascular Comorbidities in a Predominantly African-American Population with COVID-19. medRxiv 2020; : 2020.06.28.20141929.

201 Melo AC de, Thuler LCS, Silva JL da, et al. Cancer inpatient with COVID-19: a report from the Brazilian National Cancer Institute. medRxiv 2020; : 2020.06.27.20141499. 
202 Auvinen R, Nohynek H, Syrjänen R, et al. Comparison of the clinical characteristics and outcomes of hospitalized adult COVID-19 and influenza patients: a prospective observational study. medRxiv 2020; : 2020.06.29.20140632.

203 Souza FSH, Hojo-Souza NS, Santos EB, Silva CM, Guidoni DL. Predicting the disease outcome in COVID-19 positive patients through Machine Learning: a retrospective cohort study with Brazilian data. medRxiv 2020; : 2020.06.26.20140764.

204 Mendy A, Apewokin S, Wells AA, Morrow AL. Factors Associated with Hospitalization and Disease Severity in a Racially and Ethnically Diverse Population of COVID-19 Patients. medRxiv 2020; : 2020.06.25.20137323.

205 Pongpirul WA, Wiboonchutikul S, Charoenpong L, et al. Clinical course and potential predicting factors of pneumonia of adult patients with coronavirus disease 2019 (COVID-19): A retrospective observational analysis of 193 confirmed cases in Thailand. medRxiv 2020; : 2020.06.24.20139642.

206 Jin C, Gu J, Yuan Y, et al. Treatment of Six COVID-19 Patients with Convalescent Plasma. medRxiv 2020; : 2020.05.21.20109512.

207 Fisman D, Greer AL, Tuite A. Derivation and Validation of Clinical Prediction Rule for COVID-19 Mortality in Ontario, Canada. medRxiv 2020; : 2020.06.21.20136929.

208 Madariaga MLL, Guthmiller J, Schrantz S, et al. Clinical predictors of donor antibody titer and correlation with recipient antibody response in a COVID-19 convalescent plasma clinical trial. medRxiv 2020; : 2020.06.21.20132944. 209 Senkal N. Chronic ACE Inhibitor use is Associated with Decreased Odds of Severe Disease in Patients with COVID-19. Anatol J Cardiol 2020. DOI:10.14744/AnatolJCardiol.2020.57431.

210 Mohamud AY, Griffith B, Rehman M, et al. Intraluminal Carotid Artery Thrombus in COVID-19: Another Danger of Cytokine Storm? Am J Neuroradiol 2020; published online July 2. DOl:10.3174/ajnr.A6674.

211 Magleby R, Westblade LF, Trzebucki A, et al. Impact of SARS-CoV-2 Viral Load on Risk of Intubation and Mortality Among Hospitalized Patients with Coronavirus Disease 2019. Clin Infect Dis DOI:10.1093/cid/ciaa851.

212 Kimmig LM, Wu D, Gold M, et al. IL6 inhibition in critically ill COVID-19 patients is associated with increased secondary infections. medRxiv 2020; : 2020.05.15.20103531.

213 Bello-Chavolla OY, Antonio-Villa NE, Vargas-Vázquez A, Fermín-Martínez CA, Márquez-Salinas A, Bahena-López JP. Profiling pre-symptomatic and asymptomatic cases with confirmed SARS-CoV-2 infection in Mexico City. medRxiv 2020; : 2020.07.02.20145516.

214 Zacharioudakis IM, Prasad PJ, Zervou FN, et al. Association of SARS-CoV-2 Genomic Load with COVID-19 Patient Outcomes. medRxiv 2020; : 2020.07.02.20145151.

215 Antonio-Villa NE, Bello-Chavolla OY, Vargas-Vazquez A, Fermin-Martinez CA, Marquez-Salinas A, Bahena-Lopez JP. Health-care workers with COVID-19 living in Mexico City: clinical characterization and related outcomes. medRxiv $2020 ;:$ : 2020.07.02.20145169.

216 Patel M, Chowdhury J, Mills N, et al. ROX Index Predicts Intubation in Patients with COVID-19 Pneumonia and Moderate to Severe Hypoxemic Respiratory Failure Receiving High Flow Nasal Therapy. medRxiv 2020; : 2020.06.30.20143867.

217 Merzon E, Tworowski D, Gorohovski A, et al. Low plasma 25(OH) vitamin D3 level is associated with increased risk of COVID-19 infection: an Israeli population-based study. medRxiv 2020; : 2020.07.01.20144329. 
218 Fan X, Yin C, Wang J, et al. Pre-diagnostic circulating concentrations of insulin-like growth factor-1 and risk of COVID-19 mortality: results from UK Biobank. medRxiv 2020; : 2020.07.09.20149369.

219 Shi Z, Resurreccion WK, Wang C-H, et al. Association of Cancer with Risk and Mortality of COVID-19: Results from the UK Biobank. medRxiv 2020; : 2020.07.10.20151076.

220 Maucourant C, Filipovic I, Ponzetta A, et al. Natural killer cell activation related to clinical outcome of COVID-19. medRxiv 2020; : 2020.07.07.20148478.

221 Elmunzer BJ, Spitzer RL, Foster LD, et al. Digestive Manifestations in Patients Hospitalized with COVID-19. medRxiv 2020; : 2020.07.07.20143024.

222 Alizadehsani R, Sani ZA, Behjati M, et al. Risk Factors Prediction, Clinical Outcomes, and Mortality of COVID-19 Patients. medRxiv 2020; : 2020.07.07.20148569.

223 Xie Y, Chen S, Wang X, et al. Early Diagnosis and Clinical Significance of Acute Cardiac Injury - Under the Iceberg: A Retrospective Cohort Study of 619 Non-critically III Hospitalized COVID-19 Pneumonia Patients. medRxiv 2020; : 2020.07.06.20147256.

224 Fox TA, Troy-Barnes E, Kirkwood AA, et al. Clinical outcomes and risk factors for severe COVID-19 infection in patients with haematological disorders receiving chemo- or immunotherapy. Br J Haematol; n/a. DOI:10.1111/bjh.17027.

225 Martinez-Resendez MF, Castilleja-Leal F, Torres-Quintanilla A, et al. Initial experience in Mexico with convalescent plasma in COVID-19 patients with severe respiratory failure, a retrospective case series. medRxiv 2020; : 2020.07.14.20144469.

226 Hoertel N, Rico MS, Vernet R, et al.Observational Study of Haloperidol in Hospitalized Patients with Covid-19. medRxiv 2020; : 2020.07.15.20150490.

227 McGrail DE, Edwards D. COVID-19 Case Series at UnityPoint Health St. Lukes Hospital in Cedar Rapids, IA. medRxiv 2020; : 2020.07.17.20156521.

228 Pandolfi L, Fossali T, Frangipane V, et al. Broncho-alveolar inflammation in COVID-19 patients: a correlation with clinical outcome. medRxiv 2020; : 2020.07.17.20155978.

229 Kazuyoshi Kurashima, Naho Kagiyama, Takashi Ishiguro, et al. IgG antibody seroconversion and the clinical progression of COVID-19 pneumonia: A retrospective, cohort study | medRxiv. https://doi.org/10.1101/2020.07.16.20154088 (accessed Aug 25, 2020).

230 Zhan Z, Yang X, Du H, et al. Early Improvement of Acute Respiratory Distress Syndrome in Patients with COVID19: Insights from the Data of ICU Patients in Chongqing, China. medRxiv 2020; : 2020.07.15.20154047.

231 Omrani AS, Almaslamani MA, Daghfal J, et al. The First Consecutive 5000 Patients with Coronavirus Disease 2019 from Qatar; a Nation-wide Cohort Study. medRxiv 2020; : 2020.07.15.20154690.

232 Gupta R, Agrawal R, Bukhari Z, et al. Higher Comorbidities and Early Death is Characteristic of Hospitalized African-American Patients with COVID-19. medRxiv 2020; : 2020.07.15.20154906.

233 Hussein MH, Toraih EA, Attia AS, et al. Asthma in COVID-19: An extra chain fitting around the neck? medRxiv $2020 ;: 2020.07 .13 .20153130$.

234 Bian H, Zheng Z-H, Wei D, et al. Meplazumab treats COVID-19 pneumonia: an open-labelled, concurrent controlled add-on clinical trial. medRxiv 2020; : 2020.03.21.20040691. 
235 Eiros R, Barreiro-Perez M, Martin-Garcia A, et al. Pericarditis and myocarditis long after SARS-CoV-2 infection: a cross-sectional descriptive study in health-care workers. medRxiv 2020; : 2020.07.12.20151316.

236 Marcos M, Belhassen-Garcia M, Puente AS-, et al. Development of a severity of disease score and classification model by machine learning for hospitalized COVID-19 patients. medRxiv 2020; : 2020.07.13.20150177.

237 Hoertel N, Rico MS, Vernet R, et al.Association between SSRI Antidepressant Use and Reduced Risk of Intubation or Death in Hospitalized Patients with Coronavirus Disease 2019: a Multicenter Retrospective Observational Study. medRxiv 2020; : 2020.07.09.20143339.

238 Soares R de CM, Mattos LR, Raposo LM. Risk Factors for Hospitalization and Mortality due to COVID-19 in Espírito Santo State, Brazil. 2020; : tpmd200483.

239 Zobairy H, Shamsoddin E, Rasouli MA, et al. Association of olfactory dysfunction with hospitalization for COVID-19: a multicenter study in Kurdistan. medRxiv 2020; : 2020.07.26.20158550.

240 Altamimi H, Alahmad Y, Khazal F, et al. The Outcome of COVID-19 Patients with Acute Myocardial Infarction. medRxiv 2020; : 2020.07.21.20156349.

241 Thompson JV, Meghani N, Powell BM, et al. Patient characteristics and predictors of mortality in 470 adults admitted to a district general hospital in England with Covid-19. medRxiv 2020; : 2020.07.21.20153650.

242 Reiter T, Pajenda S, Wagner L, et al.Covid-19 serology in nephrology health care workers. medRxiv 2020; : 2020.07.21.20136218.

243 Motta JK, Ogunnaike RO, Shah R, et al.Clinical Outcomes With the Use of Prophylactic Versus Therapeutic Anticoagulation in COVID-19. medRxiv 2020; : 2020.07.20.20147769.

244 Santos C, Rhee Y, Hollinger E, et al.Comparative Incidence and Outcomes of COVID-19 in Kidney or KidneyPancreas Transplant Recipients Versus Kidney or Kidney-Pancreas Waitlisted Patients: A Pilot Study. medRxiv 2020; : 2020.07.20.20157990.

245 Schneeweiss MC, Leonard S, Weckstein A, Schneeweiss S, Rassen J. Renin-Angiotensin-Aldosterone-System inhibitor use in patients with COVID-19 infection and prevention of serious events: a cohort study in commercially insured patients in the US. medRxiv 2020; : 2020.07.22.20159855.

246 Concha-Mejia A, Rincon-Sanchez RA. CCOFEE-GI Study: Colombian COVID19 First Experience in Gastroentrology. Characterization of digestive manifestations in patients diagnosed with COVID-19 at a highly complex institution in Bogota D.C., Colombia. medRxiv 2020; : 2020.07.24.20161604.

247 Izquierdo JL, Almonacid C, Gonzalez Y, et al. The impact of COVID-19 on patients with asthma. medRxiv 2020; : 2020.07.24.20161596.

248 Bernaola N, Mena R, Bernaola A, et al.Observational Study of the Efficiency of Treatments in Patients Hospitalized with Covid-19 in Madrid. medRxiv 2020; : 2020.07.17.20155960.

249 Qi D, Yan X, Tang X, et al. Epidemiological and clinical features of 2019-nCoV acute respiratory disease cases in Chongqing municipality, China: a retrospective, descriptive, multiple-center study. medRxiv 2020; : 2020.03.01.20029397. 250 Peters EJ, Collard D, Assen S van, et al. Outcomes of Persons With COVID-19 in Hospitals With and Without Standard Treatment With (Hydroxy)chloroquine. medRxiv 2020; : 2020.08.14.20173369.

251 Ouyang J, Shan X, Wang X, et al. Clinical characteristics of COVID-19 and the model for predicting the occurrence 
of critically ill patients: a retrospective cohort study. medRxiv 2020; : 2020.08.13.20173799.

252 Valenzuela O, Ibanez SE, Poli M, et al.First report of tocilizumab use in a cohort of Latin American patients hospitalized for severe COVID-19 pneumonia. medRxiv 2020; : 2020.08.12.20173104.

253 Monteiro ACC, Suri R, Emeruwa IO, et al. Obesity and Smoking as Risk Factors for Invasive Mechanical Ventilation in COVID-19: a Retrospective, Observational Cohort Study. medRxiv 2020; : 2020.08.12.20173849.

254 Philipose Z, Smati N, Wong CSJ, Aspey K, Mendall MA. Obesity, old age and frailty are the true risk factors for COVID-19 mortality and not chronic disease or ethnicity in Croydon. medRxiv 2020; : 2020.08.12.20156257.

255 Weerahandi H, Hochman KA, Simon E, et al. Post-discharge health status and symptoms in patients with severe COVID-19. medRxiv 2020; : 2020.08.11.20172742.

256 Altibi AM, Bhargava P, Liaqat $\mathrm{H}$, et al. Comparative Clinical Outcomes and Mortality in Prisoner and Non-Prisoner Populations Hospitalized with COVID-19: A Cohort from Michigan. medRxiv 2020; : 2020.08.08.20170787.

257 Izzi-Engbeaya C, Distaso W, Amin A, et al. Severe COVID-19 and Diabetes: A Retrospective Cohort Study from Three London Teaching Hospitals. medRxiv 2020; : 2020.08.07.20160275.

258 Rizzo S, Chawla D, Zalocusky K, et al. Descriptive epidemiology of 16,780 hospitalized COVID-19 patients in the United States. medRxiv 2020; : 2020.07.17.20156265.

259 Dashti HT, Bates D, Fiskio JM, Roche EC, Mora S, Demler O. Clinical Characteristics and Severity of COVID-19 Disease in Patients from Boston Area Hospitals. medRxiv 2020; : 2020.07.27.20163071.

260 Morshed MS, Mosabbir AA, Chowdhury P, Ashadullah SM, Hossain MS. Clinical manifestations of patients with Coronavirus Disease 2019 (COVID- 19) attending at hospitals in Bangladesh. medRxiv 2020; : 2020.07.30.20165100. 261 Jun T, Nirenberg S, Kovatch P, Huang K. Sex-specificity of mortality risk factors among hospitalized COVID-19 patients in New York City: prospective cohort study. medRxiv 2020; : 2020.07.29.20164640.

262 Higuchi T, Nishida T, Iwahashi H, et al. Early Clinical Factors Predicting the Development of Critical Disease in Japanese Patients with COVID-19: A Single-Center Retrospective, Observational Study. medRxiv 2020; : 2020.07.29.20159442.

263 Zhou K, Sun Y, Li L, et al. Eleven Routine Clinical Features Predict COVID-19 Severity. medRxiv 2020; : 2020.07.28.20163022.

264 Salerno S, Zhao Z, Sankar SP, et al. Understanding the patterns of repeated testing for COVID-19: Association with patient characteristics and outcomes. medRxiv 2020; : 2020.07.26.20162453.

265 Kumar A, Prasad G, Srivastav S, Gautam VK, Sharma N. A Retrospective Study on Efficacy and Safety of Guduchi Ghan Vati for Covid-19 Asymptomatic Patients. medRxiv 2020; : 2020.07.23.20160424.

266 Hao S-R, Zhang S-Y, Lian J-S, et al. Liver Enzyme Elevation in Coronavirus Disease 2019: A Multicenter, Retrospective, Cross-Sectional Study. Am J Gastroenterol 2020; published online June 1.

DOI:10.14309/ajg.0000000000000717.

267 Iversen K, Bundgaard H, Hasselbalch RB, et al. Risk of COVID-19 in health-care workers in Denmark: an observational cohort study. Lancet Infect Dis 2020; 0. DOI:10.1016/S1473-3099(20)30589-2.

268 Hippisley-Cox J, Young D, Coupland C, et al. Risk of severe COVID-19 disease with ACE inhibitors and angiotensin receptor blockers: cohort study including 8.3 million people. Heart 2020; : heartjnl-2020-317393. 
269 Fillmore NR, La J, Szalat RE, et al. Prevalence and outcome of Covid-19 infection in cancer patients: a national VA study. medRxiv 2020; : 2020.08.21.20177923.

270 Rashid M, Wu J, Timmis A, et al. Clinical Characteristics and Outcomes of COVID-19 Positive Acute Coronary Syndrome Patients; a multisource Electronic Healthcare Records Study from England. medRxiv 2020; : 2020.08.20.20175091.

271 Pan A, Khan O, Meeks J, et al. Disparities in COVID-19 Hospitalizations and Mortality among Black and Hispanic Patients: Cross-Sectional Analysis from the Greater Houston Metropolitan Area. medRxiv 2020; : 2020.08.19.20177956. 272 Alkurt G, Murt A, Aydin Z, et al. Seroprevalence of Coronavirus Disease 2019 (COVID-19) Among Health Care Workers from Three Pandemic Hospitals of Turkey. medRxiv 2020; : 2020.08.19.20178095.

273 Zhao Z, Chen A, Hou W, et al. Prediction model and risk scores of ICU admission and mortality in COVID-19. PLOS ONE 2020; 15: e0236618.

274 Holman N, Knighton P, Kar P, et al. Risk factors for COVID-19-related mortality in people with type 1 and type 2 diabetes in England: a population-based cohort study. Lancet Diabetes Endocrinol 2020; 0. DOI:10.1016/S22138587(20)30271-0.

275 Qu J, Chang LK, Tang X, et al. Clinical characteristics of COVID-19 and its comparison with influenza pneumonia. Acta Clin Belg 2020; 0: 1-9.

276 Chand S, Kapoor S, Orsi D, et al. COVID-19-Associated Critical Illness—Report of the First 300 Patients Admitted to Intensive Care Units at a New York City Medical Center: J Intensive Care Med 2020; published online Aug 19. DOI:10.1177/0885066620946692.

277 Oliveira E, Parikh A, Lopez-Ruiz A, et al. ICU Outcomes and Survival in Patients with Severe COVID-19 in the Largest Health Care System in Central Florida. medRxiv 2020; : 2020.08.25.20181909.

278 Mohamed-Hussein A, Galal I, Saad M, et al. Post-COVID-19 Functional Status: Relation to age, smoking, hospitalization and comorbidities. medRxiv 2020; : 2020.08.26.20182618.

279 Villar-Garcia J, Vivanco-Hidalgo RM, Cleries M, et al. Risk factors for SARS-CoV-2 infection, hospitalisation, and death in Catalonia, Spain: a population-based cross-sectional study. medRxiv 2020; : 2020.08.26.20182303.

280 Ibarra-Nava I, Flores-Rodriguez KG, Ruiz-Herrera V, et al. Ethnic disparities in COVID-19 mortality in Mexico: a cross-sectional study based on national data. medRxiv 2020; : 2020.08.26.20182543.

281 Ibrahim D, Dulipsingh L, Zapatka L, et al. Factors Associated with Good Patient Outcomes Following Convalescent Plasma in COVID-19: A Prospective Phase II Clinical Trial. medRxiv 2020; : 2020.08.27.20183293.

282 Rubio-Rivas M, Ronda M, Padulles A, et al. Beneficial Effect of Corticosteroids in Preventing Mortality in Patients Receiving Tocilizumab to Treat Severe COVID-19 IIIness. medRxiv 2020; : 2020.08.31.20182428.

283 Mamtani M, Athavale AM, Abraham M, et al. ASSOCIATION OF HYPERGLYCEMIA WITH HOSPITAL MORTALITY IN COVID-19 PATIENTS WITHOUT DIABETES: A COHORT STUDY. medRxiv 2020; : 2020.08.31.20185157.

284 Ren HG, Guo X, Blighe K, et al. Risk Factors for ICU Admission, Mechanical Ventilation and Mortality in Hospitalized Patients with COVID-19 in Hubei, China. medRxiv 2020; : 2020.08.31.20184952.

285 Yoo E, Percha B, Tomlinson M, et al. Development and calibration of a simple mortality risk score for hospitalized 
COVID-19 adults. medRxiv 2020; : 2020.08.31.20185363.

286 Mutambudzi M, Niedzwiedz CL, Macdonald EB, et al. Occupation and risk of severe COVID-19: prospective cohort study of 120,075 UK Biobank participants. medRxiv 2020; : 2020.05.22.20109892.

287 Yan H, Valdes AM, Vijay A, et al. Role of Drugs used for chronic disease management on Susceptibility and Severity of COVID-19: A Large Case-Control Study. medRxiv 2020; : 2020.04.24.20077875.

288 Mancilla-Galindo J, Vera-Zertuche JM, Navarro-Cruz AR, et al. Development and Validation of the Patient History COVID-19 (PH-Covid19) Scoring System: A Multivariable Prediction Model of Death in Mexican Patients with COVID-19. medRxiv 2020; : 2020.09.05.20189142.

289 Ullah AZMD, Sivapalan L, Chelala C, Kocher HM. COVID-19 in patients with hepatobiliary and pancreatic diseases in East London: A single-centre cohort study. medRxiv 2020; : 2020.09.07.20189621.

290 Nicholson CJ, Wooster L, Sigurslid HH, et al. Estimating Risk of Mechanical Ventilation and Mortality Among Adult COVID-19 patients Admitted to Mass General Brigham: The VICE and DICE Scores. medRxiv 2020; : 2020.09.14.20194670.

291 Ariza BE, Torres YX, Salgado D, et al. Seroprevalence and seroconversion rates to SARS-CoV-2 in interns, residents, and medical doctors in a University Hospital in Bogota, Colombia. medRxiv 2020; : 2020.09.15.20195313. 292 Zhu S, Gao Q, Yang L, et al. Prevalence and risk factors of disability and anxiety in a retrospective cohort of 432 survivors of Coronavirus Disease-2019 (Covid-19) from China. medRxiv 2020; : 2020.08.26.20182246.

293 Sun L, Sanjna S, Le A, et al. Rates of COVID-19-related Outcomes in Cancer compared to non-Cancer Patients. medRxiv 2020; : 2020.08.14.20174961.

294 Kalan ME, Ghobadi H, Taleb ZB, et al. Descriptive characteristics of hospitalized adult smokers and never-smokers with COVID-19. Tob Induc Dis 2020; 18. DOI:10.18332/tid/122759.

295 Burrell AJ, Pellegrini B, Salimi F, et al. Outcomes of COVID-19 patients admitted to Australian intensive care units during the early phase of the pandemic. Med J Aust 2020; : 1.

296 Meini S, Fortini A, Andreini R, Sechi LA, Tascini C. The Paradox of the Low Prevalence of Current Smokers Among Covid-19 Patients Hospitalized in Non-Intensive Care Wards: Results From an Italian Multicenter Case-Control Study. Nicotine Tob Res DOI:10.1093/ntr/ntaa188.

297 Favara DM, Cooke A, Doffinger R, et al. First results from the UK COVID-19 Serology in Oncology Staff Study (CSOS). medRxiv 2020; : 2020.06.22.20136838.

298 da Silva Neto PV, de Carvalho JCS, Pimentel VE, et al. Prognostic value of sTREM-1 in COVID-19 patients: a biomarker for disease severity and mortality. Infectious Diseases (except HIV/AIDS), 2020

DOI:10.1101/2020.09.22.20199703.

299 Li F, Cai Y, Gao C, et al. Clinical Course And Risk Factors For In-hospital Death In Critical COVID-19 In Wuhan, China. Public and Global Health, 2020 DOl:10.1101/2020.09.26.20189522.

300 Cardiovascular disease and severe hypoxemia associated with higher rates of non-invasive respiratory support failure in COVID-19. ;: 47.

301 Lopez-Medrano F, Perez-Jacoiste Asin MA, Fernandez-Ruiz M, et al. Combination therapy with tocilizumab and corticosteroids for aged patients with severe COVID-19 pneumonia: a single-center retrospective study. Infectious 
Diseases (except HIV/AIDS), 2020 DOI:10.1101/2020.09.26.20202283.

302 Incerti D, Rizzo S, Li X, et al. Risk factors for mortality among hospitalized patients with COVID-19. Infectious Diseases (except HIV/AIDS), 2020 DOI:10.1101/2020.09.22.20196204.

303 Collard D, Nurmohamed NS, Kaiser Y, et al. Cardiovascular risk factors are independently associated with COVID19 mortality: a prospective cohort study. Infectious Diseases (except HIV/AIDS), 2020 DOI:10.1101/2020.10.01.20205229. 304 Robinson LB, Wang L, Fu X. COVID-19 severity in asthma patients: A multi-center matched cohort study. ; : 27. 305 Erber J, Kappler V, Haller B, et al.Strategies for infection control and prevalence of anti-SARS-CoV-2 IgG in 4,554 employees of a university hospital in Munich, Germany. Epidemiology, 2020 DOI:10.1101/2020.10.04.20206136.

306 Chaudhary A, Singh UN, Paudel P, et al. Characteristics and outcomes of hospitalized adults with COVID-19 in Nepal: a multicenter, prospective cohort study. medRxiv 2020; : 2020.10.03.20206128.

307 Nesbitt R. High seroprevalence of SARS-CoV-2 antibodies among people living in precarious situations in lle de France. 2020; : 25.

308 Savarraj JP, Burkett AB, Hinds SN, et al. Three-month outcomes in hospitalized COVID-19 patients. ; : 7.

309 Israel A, Schäffer AA, Cicurel A, et al. Large population study identifies drugs associated with reduced COVID-19 severity. medRxiv 2020; : 2020.10.13.20211953.

310 El-Solh AA, Meduri UG, Lawson Y, Carter M, Mergenhagen KA. CLINICAL COURSE AND OUTCOME OF COVID19 ACUTE RESPIRATORY DISTRESS SYNDROME: DATA FROM A NATIONAL REPOSITORY. medRXIV 2020; : 2020.10.16.20214130.

311 Chudasama YV, Zaccardi F, Gillies CL, et al. Patterns of Multimorbidity and Risk of Severe SARS-CoV-2 Infection: an observational study in the U.K. Epidemiology, 2020 DOI:10.1101/2020.10.21.20216721.

312 Salama C, Han J, Yau L, et al. Tocilizumab in nonventilated patients hospitalized with Covid-19 pneumonia. ; : 34. 313 Makaronidis J, Mok J, Balogun N, et al.Seroprevalence of SARS-CoV-2 antibodies in people with an acute loss in their sense of smell and/or taste in a community-based population in London, UK: An observational cohort study. PLOS Med 2020; 17: e1003358.

314 Anwer PR Balachandar Kathirvelu, Abhishek Chakraborti, Mahesh Gajendran, Umar Zhahid, Snigdha Ghanta, Ifeanyichkwu Onukogu, Joshua Tetteh Narh, Jen C Wang, Faiz. COVID-19 in Cancer Patients From New York City: A Comparative Single Center Retrospective Analysis - Preethi Ramachandran, Balachandar Kathirvelu, Abhishek Chakraborti, Mahesh Gajendran, Umar Zhahid, Snigdha Ghanta, Ifeanyichkwu Onukogu, Joshua Tetteh Narh, Jen C. Wang, Faiz Anwer, 2020. Cancer Control 2020; published online Oct 12. https://journals.sagepub.com/doi/10.1177/1073274820960457 (accessed Oct 28, 2020). 315 Hadi YB, Naqvi SFZ, Kupec JT, Sarwari AR. Characteristics and outcomes of COVID-19 in patients with HIV: a multicentre research network study. AIDS 2020; 34: F3-8.

316 Luo J, Rizvi H, Preeshagul IR, et al. COVID-19 in patients with lung cancer. Ann Oncol 2020; 31: 1386-96.

317 Ioannou GN, Locke E, Green P, et al. Risk Factors for Hospitalization, Mechanical Ventilation, or Death Among 10131 US Veterans With SARS-CoV-2 Infection. JAMA Netw Open 2020; 3: e2022310.

318 Pritchard M, Dankwa EA, Hall M, et al. ISARIC Clinical Data Report 4 October 2020. medRxiv 2020; : 2020.07.17.20155218. 
319 Perico L, Tomasoni S, Peracchi T, et al. COVID-19 and lombardy: TESTing the impact of the first wave of the pandemic. EBioMedicine 2020; 61. DOI:10.1016/j.ebiom.2020.103069.

320 Lamure S, Duléry R, Blasi RD, et al. Determinants of outcome in Covid-19 hospitalized patients with lymphoma: A retrospective multicentric cohort study. EClinicalMedicine 2020; 27. DOI:10.1016/j.eclinm.2020.100549.

321 Yadaw AS, Li Y, Bose S, lyengar R, Bunyavanich S, Pandey G. Clinical features of COVID-19 mortality: development and validation of a clinical prediction model. Lancet Digit Health 2020; 2: e516-25.

322 Zinellu A, Arru F, De Vito A, et al. The De Ritis ratio as prognostic biomarker of in-hospital mortality in COVID-19 patients. Eur J Clin Invest2021; 51. DOI:10.1111/eci.13427.

323 Ziehr DR, Alladina J, Petri CR, et al.Respiratory Pathophysiology of Mechanically Ventilated Patients with COVID19: A Cohort Study. Am J Respir Crit Care Med 2020; 201: 1560-4.

324 Zhou Y, He Y, Yang H, et al. Exploiting an early warning Nomogram for predicting the risk of ICU admission in patients with COVID-19: a multi-center study in China. Scand J Trauma Resusc Emerg Med 2020; 28: 106.

325 Zhou W, Song L, Wang X, et al. Cardiac injury prediction and lymphocyte immunity and inflammation analysis in hospitalized patients with coronavirus disease 2019 (COVID-19). Int J Cardiol 2020; : S016752732034002X.

326 Zhou W, Qin X, Hu X, Lu Y, Pan J. Prognosis models for severe and critical COVID-19 based on the Charlson and Elixhauser comorbidity indices. Int J Med Sci 2020; 17: 2257-63.

327 Zhang X, Li X, Sun Z, et al. Physical activity and COVID-19: an observational and Mendelian randomisation study. J Glob Health 2020; 10: 020514.

328 Zhan T, Liu M, Tang Y, et al. Retrospective analysis of clinical characteristics of 405 patients with COVID-19. J Int Med Res 2020; 48: 030006052094903.

329 Wang Z-H, Shu C, Ran X, Xie C-H, Zhang L. Critically III Patients with Coronavirus Disease 2019 in a Designated ICU: Clinical Features and Predictors for Mortality. Risk Manag Healthc Policy 2020; Volume 13: 833-45.

330 Wang Z, Zheutlin A, Kao Y-H, et al. Hospitalised COVID-19 patients of the Mount Sinai Health System: a retrospective observational study using the electronic medical records. BMJ Open 2020; 10: e040441.

331 Vila-Córcoles A, Ochoa-Gondar O, Satué-Gracia EM, et al. Influence of prior comorbidities and chronic medications use on the risk of COVID-19 in adults: a population-based cohort study in Tarragona, Spain. BMJ Open 2020; 10: e041577.

332 Torres-Macho J, Ryan P, Valencia J, et al. The PANDEMYC Score. An Easily Applicable and Interpretable Model for Predicting Mortality Associated With COVID-19. J Clin Med 2020; 9: 3066.

333 Tao P-Y, Leng L, Liu K, et al. Determination of risk factors for predicting the onset of symptoms in asymptomatic COVID-19 infected patients. Int J Med Sci 2020; 17: 2187-93.

334 Talavera B, García-Azorín D, Martínez-Pías E, et al. Anosmia is associated with lower in-hospital mortality in COVID-19. J Neurol Sci 2020; 419: 117163.

335 Serling-Boyd N, D’Silva KM, Hsu TY, et al. Coronavirus disease 2019 outcomes among patients with rheumatic diseases 6 months into the pandemic. Ann Rheum Dis 2020; : annrheumdis-2020-219279.

336 Raines AM, Tock JL, McGrew SJ, et al. Correlates of death among SARS-CoV-2 positive veterans: The contribution of lifetime tobacco use. Addict Behav 2021; 113: 106692. 
337 Parra-Bracamonte GM, Lopez-Villalobos N, Parra-Bracamonte FE. Clinical characteristics and risk factors for mortality of patients with COVID-19 in a large data set from Mexico. Ann Epidemiol 2020; 52: 93-98.e2.

338 O'Reilly GM, Mitchell RD, Mitra B, et al. Epidemiology and clinical features of emergency department patients with suspected and confirmed COVID -19: A multisite report from the COVID-19 Emergency Department Quality Improvement Project for July 2020 ( COVED -3). Emerg Med Australas 2020; : 1742-6723.13651.

339 Martini F, D’Alessio A, Bracchi F, et al. On Cancer, COVID-19, and CT Scans: A Monocentric Retrospective Study. J Clin Med 2020; 9: 3935.

340 Li J, Long X, Zhang Q, et al. Tobacco smoking confers risk for severe COVID-19 unexplainable by pulmonary imaging. J Intern Med 2020; : joim.13190.

341 Lassale C, Gaye B, Hamer M, Gale CR, Batty GD. Ethnic disparities in hospitalisation for COVID-19 in England: The role of socioeconomic factors, mental health, and inflammatory and pro-inflammatory factors in a community-based cohort study. Brain Behav Immun 2020; 88: 44-9.

342 Klang E, Soffer S, Nadkarni G, et al.Sex Differences in Age and Comorbidities for COVID-19 Mortality in Urban New York City. SN Compr Clin Med 2020; 2: 1319-22.

343 Kim H-J, Han D, Kim J-H, et al. An Easy-to-Use Machine Learning Model to Predict the Prognosis of Patients With COVID-19: Retrospective Cohort Study. J Med Internet Res 2020; 22: e24225.

344 Jehi L, Ji X, Milinovich A, et al. Development and validation of a model for individualized prediction of hospitalization risk in 4,536 patients with COVID-19. PLOS ONE 2020; 15: e0237419.

345 Jakob CEM, Borgmann S, Duygu F, et al. First results of the "Lean European Open Survey on SARS-CoV-2Infected Patients (LEOSS)". Infection 2020; published online Oct 1. DOI:10.1007/s15010-020-01499-0.

346 Invernizzi A, Torre A, Parrulli S, et al. Retinal findings in patients with COVID-19: Results from the SERPICO-19 study. EClinicalMedicine 2020; 27: 100550.

347 Ilic I, Zdravkovic M, Timcic S, Unic Stojanovic D, Bojic M, Loncar G. Pneumonia in healthcare workers during a COVID-19 outbreak at a cardiovascular hospitals. Int J Infect Dis 2021; 103: 188-93.

348 Hamadah H, Alahmad B, Behbehani M, et al. COVID-19 clinical outcomes and nationality: results from a Nationwide registry in Kuwait. BMC Public Health 2020; 20: 1384.

349 Gianfrancesco MA, Leykina LA, Izadi Z, et al. Race/ethnicity association with COVID-19 outcomes in rheumatic disease: Data from the COVID-19 Global Rheumatology Alliance Physician Registry. Arthritis Rheumatol 2020; : art.41567.

350 Ghinai I, Davis ES, Mayer S, et al. Risk Factors for Severe Acute Respiratory Syndrome Coronavirus 2 Infection in Homeless Shelters in Chicago, Illinois-March-May, 2020. Open Forum Infect Dis 2020; 7: ofaa477.

351 Fond G, Pauly V, Orleans V, et al. Increased in-hospital mortality from COVID-19 in patients with schizophrenia. L'Encéphale 2020; : S0013700620301883.

352 Best JH, Mohan SV, Kong AM, et al. Baseline Demographics and Clinical Characteristics Among 3471 US Patients Hospitalized with COVID-19 and Pulmonary Involvement: A Retrospective Study. Adv Ther 2020; 37: 4981-95.

353 Bellan M, Patti G, Hayden E, et al.Fatality rate and predictors of mortality in an Italian cohort of hospitalized COVID19 patients. Sci Rep 2020; 10: 20731. 
354 Alharthy A, Aletreby W, Faqihi F, et al. Clinical Characteristics and Predictors of 28-Day Mortality in 352 Critically III Patients with COVID-19: A Retrospective Study: J Epidemiol Glob Health 2020. DOI:10.2991/jegh.k.200928.001.

355 Alguwaihes AM, Al-Sofiani ME, Megdad M, et al. Diabetes and Covid-19 among hospitalized patients in Saudi Arabia: a single-centre retrospective study. Cardiovasc Diabetol 2020; 19: 205.

356 Aksu K, Naziroğlu T, Özkan P. Factors determining COVID-19 pneumonia severity in a country with routine BCG vaccination. Clin Exp Immunol 2020; 202: 220-5.

357 Adrish M, Chilimuri S, Mantri N, et al. Association of smoking status with outcomes in hospitalised patients with COVID-19. BMJ Open Respir Res 2020; 7: e000716.

358 Hoertel N, Sánchez M, Vernet R, et al.Dexamethasone use and Mortality in Hospitalized Patients with Coronavirus Disease 2019: a Multicenter Retrospective Observational Study. Infectious Diseases (except HIV/AIDS), 2020 DOI:10.1101/2020.10.23.20218172.

359 Arleo T, Tong D, Shabto J, O’Keefe G, Khosroshahi A. Clinical Course and Outcomes of coronavirus disease 2019 (COVID-19) in Rheumatic Disease Patients on Immunosuppression: A case Cohort Study at a Single Center with a Significantly Diverse Population. Epidemiology, 2020 DOI:10.1101/2020.10.26.20219154.

360 Bermejo-Martin JF, González-Rivera M, Almansa R, et al. Viral RNA load in plasma is associated with critical illness and a dysregulated host response in COVID-19. Crit Care 2020; 24: 691.

361 Joubert A, Andry F, Bertolotti A, et al. Distinguishing non severe cases of dengue from COVID-19 in the context of co-epidemics: a cohort study in a SARS-CoV-2 testing center on Reunion island. Infectious Diseases (except HIV/AIDS), 2020 DOI:10.1101/2020.10.20.20214718.

362 Kortela E, Kirjavainen V, Ahava MJ, et al. Real-life clinical sensitivity of SARS-CoV-2 RT-PCR test in symptomatic patients. Infectious Diseases (except HIV/AIDS), 2020 DOI:10.1101/2020.11.01.20223107.

363 Sourij H, Aziz F, Bräuer A, et al. COVID-19 fatality prediction in people with diabetes and prediabetes using a simple score upon hospital admission. Diabetes Obes Metab 2020; : dom.14256.

364 Galal I, Hussein AAM, Amin MT, et al.Determinants of Persistent Post COVID-19 symptoms: Value of a Novel COVID-19 symptoms score. Respiratory Medicine, 2020 DOI:10.1101/2020.11.11.20230052.

365 Clavario P, De Marzo V, Lotti R, et al. Assessment of functional capacity with cardiopulmonary exercise testing in non-severe COVID-19 patients at three months follow-up. Cardiovascular Medicine, 2020 DOI:10.1101/2020.11.15.20231985.

366 Saeed GA, AI Helali AA, Almazrouei S, Shah A, Ahmed LA. Chest CT features of COVID-19 in the region of Abu Dhabi, UAE- A single institute study. Radiology and Imaging, 2020 DOI:10.1101/2020.11.14.20229096.

367 Cadegiani FA, McCoy J, Wambier CG, Goren A. 5-Alpha-Reductase Inhibitors Reduce Remission Time of COVID19: Results From a Randomized Double Blind Placebo Controlled Interventional Trial in 130 SARS-CoV-2 Positive Men. Infectious Diseases (except HIV/AIDS), 2020 DOI:10.1101/2020.11.16.20232512.

368 Benaim AR, Sobel JA, Almog R, et al. At the dawn of winter: comparing COVID-19 and influenza presentation and trajectory. Infectious Diseases (except HIV/AIDS), 2020 DOI:10.1101/2020.11.19.20235077.

369 Singh BM, Bateman J, Viswanath A, et al. The risk of COVID hospital admission and COVID mortality during the first COVID 19 wave with a special emphasis on Ethnic Minorities: an observational study of a single, deprived, multi 
ethnic UK health economy. Infectious Diseases (except HIV/AIDS), 2020 DOI:10.1101/2020.11.20.20224691.

370 International Severe Acute Respiratory and emerging Infections Consortium, Hall M, Pritchard M, et al. ISARIC Clinical Data Report 20 November 2020. Infectious Diseases (except HIV/AIDS), 2020

DOI:10.1101/2020.07.17.20155218.

371 Márquez-Salinas A, Fermín-Martínez CA, Antonio-Villa NE, et al. Adaptive metabolic and inflammatory responses identified using accelerated aging metrics are linked to adverse outcomes in severe SARS-CoV-2 infection. Geriatric Medicine, 2020 DOI:10.1101/2020.11.03.20225375.

372 Díez-Manglano J, Marquínez MNS, García AÁ, et al. Healthcare workers hospitalized due to COVID-19 have no higher risk of death than general population. Data from the Spanish SEMI-COVID-19 Registry. Infectious Diseases (except HIV/AIDS), 2020 DOI:10.1101/2020.11.23.20236810.

373 Woolcott OO, Castilla-Bancayán JP. Diabetes and Mortality Among 1.6 Million Adult Patients Screened for SARSCoV-2 in Mexico. Infectious Diseases (except HIV/AIDS), 2020 DOI:10.1101/2020.11.25.20238345.

374 Simons D, Perski O, Shahab L, Brown J, Bailey R. The association of smoking status with hospitalisation for COVID-19 compared with other respiratory viruses a year previous: A case-control study at a single UK National Health Service trust. medRxiv 2020; : 2020.11.26.20238469.

375 Dupraz J, Butty A, Duperrex O, et al. Prevalence of SARS-CoV-2 in household members and other close contacts of COVID-19 cases: a serologic study in canton of Vaud, Switzerland. Epidemiology, 2020

DOI:10.1101/2020.11.27.20239244.

376 Chen H, Varatharajah Y, de Ramirez SS, et al. A Retrospective Longitudinal Study of COVID-19 as Seen by a Large Urban Hospital in Chicago. Infectious Diseases (except HIV/AIDS), 2020 DOI:10.1101/2020.11.29.20240606.

377 Martinez-Lacalzada M, Viteri-Noël LA, Manzano L, et al. Predicting critical illness on initial diagnosis of COVID-19: Development and validation of the PRIORITY model for outpatient applicability. Infectious Diseases (except HIV/AIDS), 2020 DOI:10.1101/2020.11.27.20237966.

378 Barasa S, Kiage-Mokaya J, Cruz-Madrid K, Friedlander M. Smoking increases the risk of COVID-19 positivity, while Never-smoking reduces the risk. Respiratory Medicine, 2020 DOI:10.1101/2020.11.30.20241380.

379 Ren HG, Guo X, Tu L, et al. Clinical Characteristics and Risk Factors for Myocardial Injury and Arrhythmia in COVID-19 patients. Cardiovascular Medicine, 2020 DOI:10.1101/2020.11.30.20190926.

380 O'Gallagher K, Shek A, Bean DM, et al. Pre-existing cardiovascular disease rather than cardiovascular risk factors drives mortality in COVID-19. Cardiovascular Medicine, 2020 DOI:10.1101/2020.12.02.20242933.

381 Modrák M, Bürkner P-C, Sieger T, et al. Detailed disease progression of 213 patients hospitalized with Covid-19 in the Czech Republic: An exploratory analysis. Infectious Diseases (except HIV/AIDS), 2020 DOI:10.1101/2020.12.03.20239863.

382 Zuo Y, Warnock M, Harbaugh A, et al. Plasma tissue plasminogen activator and plasminogen activator inhibitor-1 in hospitalized COVID-19 patients. Infectious Diseases (except HIV/AIDS), 2020 DOI:10.1101/2020.08.29.20184358.

383 Bisso IC, Huespe I, Lockhart C, et al. Clinical characteristics of critically ill patients with COVID-19. Intensive Care and Critical Care Medicine, 2020 DOI:10.1101/2020.12.09.20246413.

384 Rentsch CT, Beckman JA, Tomlinson L, et al. Early initiation of prophylactic anticoagulation for prevention of 
COVID-19 mortality: a nationwide cohort study of hospitalized patients in the United States. Infectious Diseases (except HIV/AIDS), 2020 DOI:10.1101/2020.12.09.20246579.

385 Thiabaud A, Iten A, Balmelli C, et al. SARS-CoV-2/COVID-19 hospitalised patients in Switzerland: a prospective cohort profile. Epidemiology, 2020 DOI:10.1101/2020.12.10.20246884

386 Iftime S, López-Azcona AF, Vallverdu I, et al. First and second waves of coronavirus disease-19: A comparative study in hospitalized patients in Reus, Spain. Infectious Diseases (except HIV/AIDS), 2020

DOI:10.1101/2020.12.10.20246959.

387 Vila-Corcoles A, Satue-Gracia E, Vila-Rovira A, et al. COVID-19 TARRACO Cohort Study: Development of a predictive prognostic rule for early assessment of COVID-19 patients in primary care settings. Infectious Diseases (except HIV/AIDS), 2020 DOI:10.1101/2020.12.11.20247932.

388 Lévy Y, Wiedemann A, Hejblum BP, et al. CD177, a specific marker of neutrophil activation, is a hallmark of COVID-19 severity and death. Allergy and Immunology, 2020 DOI:10.1101/2020.12.12.20246934. 